\title{
Feldinduzierte Tieftemperaturoxidation nanoskaliger Metall- und Halbleiterstrukturen
}

\author{
Dissertation \\ zur Erlangung des Doktorgrades der Mathematisch-Naturwissenschaftlichen Fakultäten \\ der Georg-August-Universität Göttingen
}

\author{
Vorgelegt von \\ CARSTEN NOWAK \\ aus Hildesheim
}

Göttingen 2008 
D7

Referent: Prof. Dr. R. Kirchheim

Korreferent: Prof. Dr. G. Schmitz

Tag der mündlichen Prüfung: 14.10.2008 


\section{Inhaltsverzeichnis}

1 Einführung 3

2 Physikalischer Hintergrund 5

2.1 Thermodynamik der Oxidation . . . . . . . . . . . . . . 5

2.1.1 Oxidation mit $\mathrm{O}_{2} \ldots \ldots \ldots \ldots \ldots$

2.1 .2 Oxidation mit $\mathrm{H}_{2} \mathrm{O} \ldots \ldots \ldots \ldots$

2.1.3 Phasendiagramme der untersuchten Modelsysteme . . . . . . . 7

2.2 Kinetik der Tieftemperaturoxidation . . . . . . . . . . . . . 7

2.2.1 Teilschritte der Oxidationsreaktion . . . . . . . . . . . 8

2.2.2 Kinetik der unbeeinflussten Tieftemperaturoxidation . . . . . . 9

2.2.3 Externe Manipulation der Tieftemperaturoxidation - Planarsysteme 11

2.2.4 Externe Manipulation der Tieftemperaturoxidation - AFM Studien 13

3 Methoden 15

3.1 Probenpräparation . . . . . . . . . . . . . . . . 15

3.1.1 Elektrochemische Präparation der W- und Al-Proben . . . . . . . 15

3.1 .2 Präparation der Si-Proben mit FIB . . . . . . . . . . . . . . 16

3.2 Experimenteller Aufbau . . . . . . . . . . . . . . . . . . . . 17

3.3 Durchführung der Oxidationsreaktion und in situ Charakterisierung . . . 18

3.4 Ex situ Charakterisierungsmethoden . . . . . . . . . . . . . . . . . . . . 19

3.4.1 Transmissionselektronenmikroskopie . . . . . . . . . . . . . . . 19

3.4.2 Atomsondentomographie . . . . . . . . . . . . . . . . . . 20

3.5 Feldberechnungen mit der Finite-Elemente-Methode . . . . . . . . . . . . 20

4 Resultate der Oxidation von W-Spitzen mit $\mathrm{H}_{2} \mathrm{O}$

4.1 Einführung des Oxidationseffekts . . . . . . . . . . . . . 23

4.2 Kinetik der Oxidationsreaktion . . . . . . . . . . . . . . 25

4.2.1 Kinetik der Morphologieentwicklung . . . . . . . . . . . . . 25

4.2 .2 Kinetik des Reaktionsstromes . . . . . . . . . . . . . . . . 28

4.3 Struktur und Stöchiometrie des Oxides . . . . . . . . . . . . . . . . . . . 29

4.3 .1 Stöchiometrie des Oxides . . . . . . . . . . . . . . . . . . . 29

4.3.2 Struktur des Oxides . . . . . . . . . . . . . . . . . . 32

4.4 Feldstärkeverteilung im Oxid . . . . . . . . . . . . . . . . . . 33

4.4.1 Feldstärkeverteilung in Abhängigkeit vom Potential . . . . . . . . 33

4.4.2 Feldstärkeverteilung in Abhängigkeit vom $\mathrm{H}_{2} \mathrm{O}$-Druck . . . . . . . 34 
5 Resultate bei Variation des Spitzenmaterials und der Gasspezies 37

5.1 Al-Spitzen . . . . . . . . . . . . . . . . . . 37

5.1.1 Kinetik der Morphologieentwicklung . . . . . . . . . . . . . 39

5.1.2 Struktur und Stöchiometrie des Oxides . . . . . . . . . . . . . . . 41

5.1.3 Feldstärkeverteilung in Abhängigkeit vom $\mathrm{H}_{2} \mathrm{O}$-Druck . . . . . . . 41

5.2 Si-Spitzen . . . . . . . . . . . . . . . . . . . . 42

5.3 Weitere Spitzenmaterialien . . . . . . . . . . . . . . . . . . . . . . . 43

5.4 Variation der Gasatmosphäre . . . . . . . . . . . . . . . . 44

6 Diskussion $\quad 45$

6.1 Kinetik und Morphologieentwicklung . . . . . . . . . . . . . . . 45

6.1.1 Kinetik des Anfangsstadiums . . . . . . . . . . . . . . . . 46

6.1.2 Morphologieentwicklung . . . . . . . . . . . . . . . 49

6.1.3 Morphologie des stabilen Zustandes . . . . . . . . . . . . . . . . . 52

6.2 Aggregatzustand des $\mathrm{H}_{2} \mathrm{O}$ auf der Spitze . . . . . . . . . . . . 53

6.3 Einfluss des Feldes auf die Oxidationsreaktion . . . . . . . . . . . . 55

6.3.1 Existenz einer kritischen Feldstärke für die Reaktion . . . . . . . 56

6.3.2 Thermodynamik der feldinduzierten Oxidationsreaktion . . . . . . 57

6.3.3 Identifikation des geschwindigkeitsbestimmenden Schrittes . . . . 58

6.4 Variation von Spitzenmaterial und Gasspezies . . . . . . . . . . . . . 60

6.4.1 Abhängigkeit der Oxidationsreaktion vom Spitzenmaterial . . . . 60

6.4.2 Abhängigkeit der Oxidationsreaktion von der Gasspezies . . . . . 62

6.5 Vergleich mit Modellen für Tieftemperaturoxidation aus der Literatur . . 62

$\begin{array}{lll}7 & \text { Zusammenfassung } & 65\end{array}$

$\begin{array}{ll}\text { Symbolverzeichnis } & 67\end{array}$

$\begin{array}{ll}\text { Literaturverzeichnis } & 71\end{array}$ 


\section{Einführung}

Die Nutzung metallischer Objekte und halbleitender Bauelemente erfolgt im alltäglichen Leben mit großer Selbstverständlichkeit. Dies mag zunächst erstaunen, da Metalle und Halbleiter in der Regel an Umgebungsatmosphäre thermodynamisch nicht stabil sind, sondern Oxide bilden. Bei Raumtemperatur ist die Kinetik der Oxidationsreaktion jedoch so langsam, dass die Oxidbildung auf oberflächennahe Bereiche beschränkt bleibt. Die Ausbildung einer Oxidschicht kann das jeweilige Material dabei effektiv vor fortschreitender Oxidation schützen. Dieser als Passivierung bezeichnete Effekt ist ursächlich für die Stabilität zahlreicher Metalle, etwa von Aluminium und rostfreien Stählen. Die Volumeneigenschaften metallischer und halbleitender Werkstoffe werden durch die Oxidbildung an der Oberfläche kaum beeinflusst. Für die Nutzung metallischer Objekte im Alltag stellt die thermodynamische Instabilität daher üblicherweise kein gravierendes Problem dar.

Beim Übergang zu nanoskaligen Systemen hingegen wird der Einfluss der Oberfläche zunehmend relevant. Die Eigenschaften "freier" nanoskaliger Systeme, etwa von Nanodrähten und Clustern, werden daher durch Oxidbildung an der Oberfläche erheblich beeinflusst. So wird etwa an Clustern aus Cobalt und Aluminium infolge der Oxidation die Ausbildung einiger nm großer Poren im Zentrum der Cluster beobachtet [Naka2007, Yin2004]. Eine kontrollierte Anwendung freier Nanostrukturen setzt daher die Kenntnis ihres Oxidationsverhaltens voraus. Dabei ist zu berücksichtigen, dass sich an nanoskaligen Systemen der Einfluss zusätzlicher Triebkräfte für die Oxidbildung besonders deutlich bemerkbar macht. Insbesondere können Unterschiede im elektrostatischen Potential einen erheblichen Einfluss ausüben: An der Oberfläche nanoskaliger Systeme treten, bedingt durch deren starke Krümmung, bereits bei moderaten Potentialdifferenzen zu einer Gegenelektrode Feldstärken in der Größenordnung von V/nm auf. Da einzelne Teilschritte der Oxidationsreaktion mit einem Ladungstransport einhergehen, resultieren daraus Energieunterschiede im Bereich von einigen eV. Diese Energieunterschiede sind von der gleichen Größenordnung wie die Bildungsenthalpie des Oxides. Daher ist zu erwarten, dass sich die Thermodynamik und die Kinetik der Oxidationsreaktion nanoskaliger Systeme unter dem Einfluss eines elektrischen Feldes erheblich ändern.

Ziel der vorliegenden Arbeit ist eine umfassende experimentelle Charakterisierung des Oxidationsverhaltens nanoskaliger Spitzen unter dem Einfluss eines elektrischen Feldes. Eine genaue Kenntnis des Oxidationsverhaltens ist dabei aus verschiedenen Gründen von Interesse: Zum einen kann eine technologische Anwendung metallischer oder halbleitender Nanostrukturen nur innerhalb der Grenzen ihrer thermodynamischen beziehungsweise kinetischen Stabilität erfolgen. Ist die Stabilität gegenüber Oxidbildung abhängig von der Stärke elektrischer Felder, so wird dadurch die Einsatzmöglichkeit von 
Nanostrukturen in elektronischen Bauelementen beschränkt. Wesentlich ist dies etwa beim Einsatz von Nanodrähten als Sensormaterial für Gase und biologische Moleküle [Cui2001, Walt2002].

Andererseits ermöglicht eine Beeinflussung der Triebkraft für die Oxidbildung durch ein elektrisches Feld eine kontrollierte Transformation von Metallen oder Halbleitern in die entsprechenden Oxide. Im Gegensatz zur Hochtemperaturoxidation tritt dabei keine thermisch aktivierte Diffusion auf, so dass eine unerwünschte Degradation nanoskaliger Strukturen durch thermische Belastung vermieden wird. Eine potentielle Anwendung der feldinduzierten Tieftemperaturoxidation liegt daher im Aufbau von Heterostrukturen oder Tunnelbarrieren [Ebin1998, Ross2008].

Schließlich liefert die Untersuchung des Feldeinflusses auf das Oxidationsverhalten verschiedener Materialien einen Einblick in die Mechanismen der Oxidationsreaktion. Durch Extrapolation kann dabei ein Vergleich mit den Modellvorstellungen zum Ablauf von Oxidationsreaktionen im feldfreien Fall vorgenommen werden. Somit lässt sich beurteilen, inwieweit die auf den Konzepten von Cabrera und Mott [Cabr1949, Mott1947] basierenden Modelle zum Ablauf der Tieftemperaturoxidation geeignet sind, auch den Einfluss externer elektrischer Felder zu berücksichtigen.

Die Grundlagen der Thermodynamik und Kinetik von Oxidationsreaktionen werden in Kapitel 2 der vorliegenden Arbeit vorgestellt. Neben einer Darstellung der etablierten Modelle zum Ablauf der Tieftemperaturoxidation liegt der Schwerpunkt dabei insbesondere auf Studien zur Manipulation von Oxidationsreaktionen durch elektrische Felder.

In Kapitel 3 werden die zur Probenpräparation und Analyse verwendeten Methoden vorgestellt. Der für die Oxidationsexperimente eingesetzte experimentelle Aufbau wird beschrieben und die Durchführung der Oxidationsexperimente geschildert. Weiterhin wird dargelegt, wie aus der Morphologie der Proben die Feldverteilung in der Umgebung der Proben mit der Finite-Elemente-Methode berechnet wird.

Die Untersuchung des Oxidationsverhaltens nanoskaliger Spitzen erfolgt im Wesentlichen an dem Modellsystem Wolfram. Ergebnisse zur Kinetik der Oxidationsreaktion, zur Struktur und Stöchiometrie des gebildeten Oxides sowie zur Feldverteilung in der Spitzenumgebung werden in Kapitel 4 präsentiert. Darüber hinaus wurden ausgewählte Untersuchungen an Proben aus Aluminium, Silizium, Molybdän, Nickel und Silber durchgeführt. Die entsprechenden Resultate werden in Kapitel 5 vorgestellt.

Eine Diskussion der Ergebnisse erfolgt in Kapitel 6. Das Oxidationsverhalten nanoskaliger Spitzen unter dem Einfluss eines elektrischen Feldes ändert sich erheblich, sofern $\mathrm{H}_{2} \mathrm{O}$ für die Oxidationsreaktion zur Verfügung steht. Auf der Grundlage der zeitlichen Entwicklung der Probenmorphologie können die wesentlichen Prozesse für den Ablauf der Oxidationsreaktion identifiziert werden. Ein Vergleich der Resultate für unterschiedliche Spitzenmaterialien zeigt, dass feldinduzierte Oxidation bei einer Vielzahl von Materialien beobachtet werden kann. 


\section{Physikalischer Hintergrund}

In diesem Kapitel wird zunächst die Thermodynamik der Oxidbildung aus einem Festkörper und einem sauerstoffenthaltenden Gas betrachtet. Da die für die Oxidationsreaktion notwendigen Diffusionsprozesse bei tiefen Temperaturen extrem langsam ablaufen, wird jedoch in der Regel der Gleichgewichtszustand nicht erreicht, sondern die Reaktion ist von einem gewissen Grad an kinetisch gehemmt. Daher wird im weiteren Verlauf des Kapitels auf die Mechanismen der Oxidationsreaktion und daraus folgende Zeitgesetze eingegangen. Abschließend wird ein Überblick über bisherige Untersuchungen zur externen Manipulation der Kinetik durch elektrische Felder gegeben.

\subsection{Thermodynamik der Oxidation}

Zunächst wird in diesem Abschnitt dargestellt, unter welchen Bedingungen die Bildung eines Oxides aus einem Element $\mathrm{M}$ und dem Gas $\mathrm{O}_{2}$ bzw. $\mathrm{H}_{2} \mathrm{O}$ thermodynamisch möglich ist. Im Anschluss werden die Phasendiagramme der untersuchten Modellsysteme W-O, $\mathrm{Al}-\mathrm{O}$ und Si-O vorgestellt.

\subsubsection{Oxidation mit $\mathrm{O}_{2}$}

Die Reaktionsgleichung für die Oxidation eines Elementes $\mathrm{M} \mathrm{zu} \mathrm{M}_{\mathrm{x}} \mathrm{O}_{\mathrm{y}}$ lässt sich in der Form

$$
\frac{2 \mathrm{x}}{\mathrm{y}} \mathrm{M}(\mathrm{s})+\mathrm{O}_{2}(\mathrm{~g}) \rightarrow \frac{2}{\mathrm{y}} \mathrm{M}_{\mathrm{x}} \mathrm{O}_{\mathrm{y}}(\mathrm{s})
$$

derart aufstellen, dass genau ein $\mathrm{O}_{2}$ Molekül reagiert. Für diese Reaktion soll nun die Änderung der Gibbsschen freien Energie $\Delta G$ des Systems bei der Temperatur $T$ und dem Sauerstoffpartialdruck $p_{\mathrm{O}_{2}}$ betrachtet werden. Da die Druckabhängigkeit des chemischen Potentials $\mu$ fester Körper vernachlässigbar ist, folgt mit dem chemischen Potential unter Standarddruck $\mu^{0}$ für die Änderung der Gibbsschen freien Energie

$$
\begin{aligned}
\Delta G\left(T, p_{\mathrm{O}_{2}}\right) & =\frac{2}{\mathrm{y}} \mu_{\mathrm{M}_{\mathrm{x}} \mathrm{O}_{\mathrm{y}}}^{0}(T)-\frac{2 \mathrm{x}}{\mathrm{y}} \mu_{\mathrm{M}}^{0}(T)-\mu_{\mathrm{O}_{2}}^{0}(T)-\mathrm{k} T \ln \left(\frac{p_{\mathrm{O}_{2}}}{\mathrm{p}^{0}}\right) \\
& =: \Delta G^{0}(T)-\mathrm{k} T \ln \left(\frac{p_{\mathrm{O}_{2}}}{\mathrm{p}^{0}}\right) .
\end{aligned}
$$

Die Oxidationsreaktion gemäß (2.1) kann folglich thermodynamisch genau dann ablaufen, wenn die Änderung der Gibbsschen freien Energie des Systems unter Standarddruck $\Delta G^{0}$ kleiner ist als der druckabhängige Term des chemischen Potentials des idealen Gases $\mathrm{k} T \ln \left(p_{\mathrm{O}_{2}} / \mathrm{p}^{0}\right)$. Eine auf Ellingham [Elli1944] zurückgehende Darstellung dieser Bedingung zeigt Abb. 2.1. Es ist unmittelbar ersichtlich, dass bei Raumtemperatur für alle 


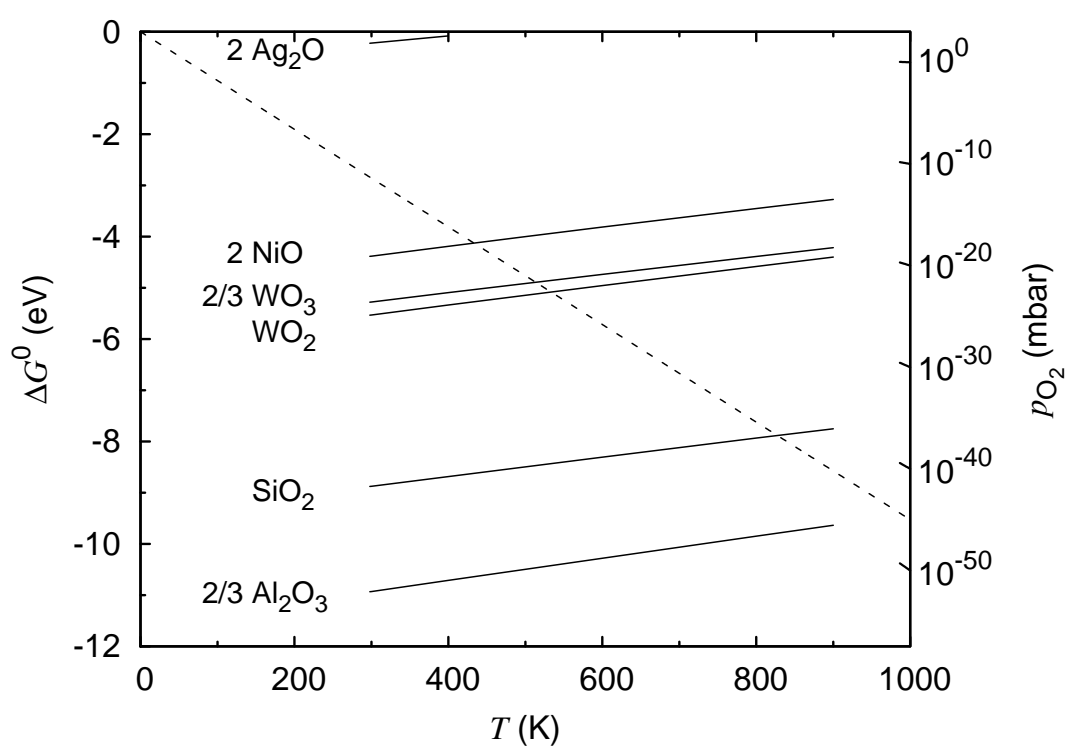

Abbildung 2.1: Ellinghamdiagramm für einige Oxide basierend auf thermodynamischen Daten aus [Knac1991]. Aufgetragen ist die Änderung der Gibbsschen freien Energie des Systems unter Standarddruck $\Delta G^{0}$ für die Oxidationsreaktion gemäß (2.1). Die sich aus dem druckabhängigen Term des chemischen Potentials des idealen Gases $\mathrm{k} T \ln \left(p_{\mathrm{O}_{2}} / \mathrm{p}^{0}\right)$ ergebenden Ursprungsgeraden sind durch die entsprechenden Druckwerte an der rechten Ordinatenachse gekennzeichnet. Die unterbrochene Linie zeigt beispielhaft die Ursprungsgerade für einen Druck von $10^{-45}$ mbar $\mathrm{H}_{2} \mathrm{O}$.

dargestellten Elemente selbst unter Ultra Hochvakuum (UHV) die Oxidbildung thermodynamisch begünstigt ist.

Da für die in Kapitel 4 dargestellten Resultate $\mathrm{H}_{2} \mathrm{O}$ als Oxidationsmittel verwendet wurde, soll im Folgenden analog zu der hier vorgenommenen Darstellung die Thermodynamik des System $\mathrm{M}-\mathrm{H}_{2} \mathrm{O}$ betrachtet werden.

\subsubsection{Oxidation mit $\mathrm{H}_{2} \mathrm{O}$}

Für die Oxidation eines Elementes $\mathrm{M}$ unter $\mathrm{H}_{2} \mathrm{O}$ Atomsphäre $\mathrm{zu}_{\mathrm{x}} \mathrm{O}_{\mathrm{y}}$ lässt sich die Reaktionsgleichung in der Form

$$
\frac{\mathrm{x}}{\mathrm{y}} \mathrm{M}(\mathrm{s})+\mathrm{H}_{2} \mathrm{O}(\mathrm{g}) \rightarrow \frac{1}{\mathrm{y}} \mathrm{M}_{\mathrm{x}} \mathrm{O}_{\mathrm{y}}(\mathrm{s})+\mathrm{H}_{2}(\mathrm{~g})
$$

derart aufstellen, dass genau ein $\mathrm{H}_{2} \mathrm{O}$ Molekül reagiert. Analog zu der Darstellung in 2.1.1 folgt für die Änderung der Gibbsschen freien Energie des Systems bei dieser Reaktion

$$
\Delta G\left(T, p_{\mathrm{H}_{2} \mathrm{O}}, p_{\mathrm{H}_{2}}\right)=\Delta G^{0}(T)-\mathrm{k} T \ln \left(\frac{p_{\mathrm{H}_{2} \mathrm{O}}}{p_{\mathrm{H}_{2}}}\right) .
$$

Aus dem zugehörigen Ellinghamdiagramm in Abb. 2.2 ist ersichtlich, dass selbst bei gleichgroßen Partialdrücken von $\mathrm{H}_{2} \mathrm{O}$ und $\mathrm{H}_{2}$ die Oxidbildung für eine Vielzahl von Elementen thermodynamisch begünstigt ist. 


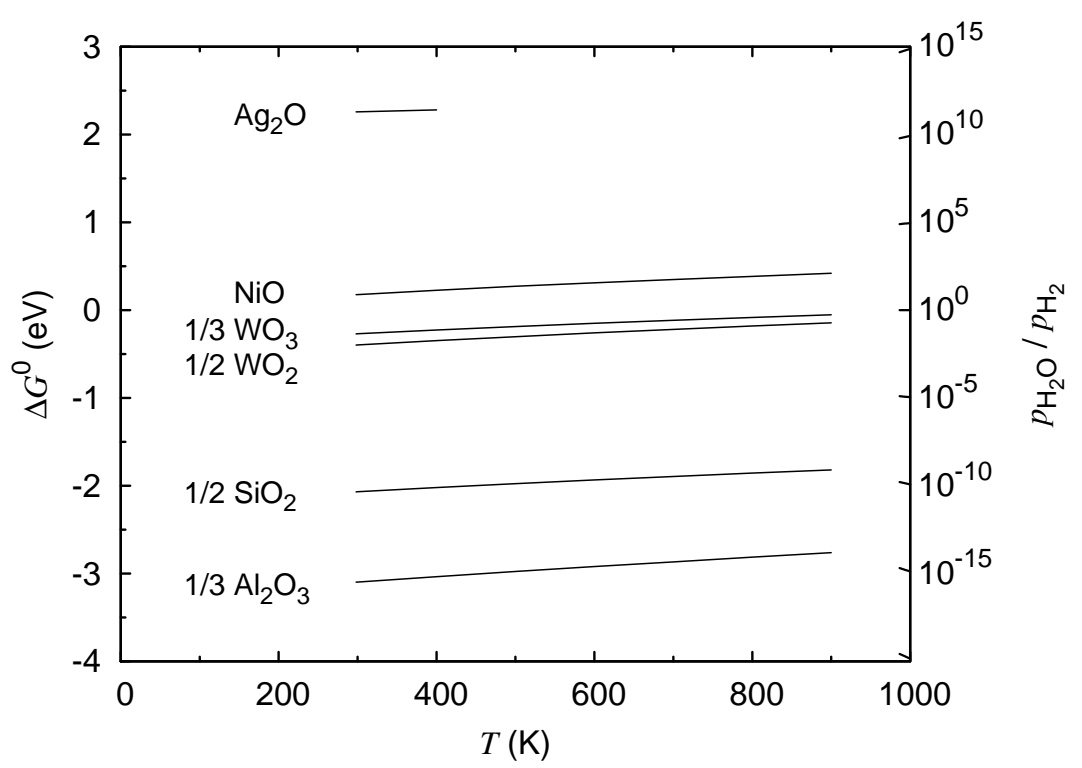

Abbildung 2.2: Ellinghamdiagramm für die Oxidationsreaktion mit $\mathrm{H}_{2} \mathrm{O}$ gemäß (2.2).

\subsubsection{Phasendiagramme der untersuchten Modelsysteme}

Das Phasendiagramm des Modellsystems W-O ist in Abb. 2.3 dargestellt. Bei tiefen Temperaturen sind lediglich die monokline $\mathrm{WO}_{2}$ - sowie die $\mathrm{WO}_{3}$-Phase, für die eine Vielzahl sehr ähnlicher Strukturinformationen vorliegt [Pred1998], stabil. Bei Temperaturen oberhalb von etwa $850 \mathrm{~K}$ sind im Konzentrationsbereich von 73,1..75,0 At.\% Sauerstoff darüberhinaus zahlreiche zum Teil metastabile Phasen gefunden worden.

Für das System Al-O existiert allein $\mathrm{Al}_{2} \mathrm{O}_{3}$ als thermodynamisch stabiles Oxid. Im Si-O System liegt ebenfalls nur $\mathrm{SiO}_{2}$ als thermodynamisch stabiles Oxid vor, $\mathrm{SiO}$ ist nach [Mass1990] lediglich eine metastabile Phase.

\subsection{Kinetik der Tieftemperaturoxidation}

In diesem Abschnitt werden zunächst die bei der Tieftemperaturoxidation ablaufenden atomistischen Prozesse sowie die daraus folgenden Zeitgesetze beschrieben. Dabei be-

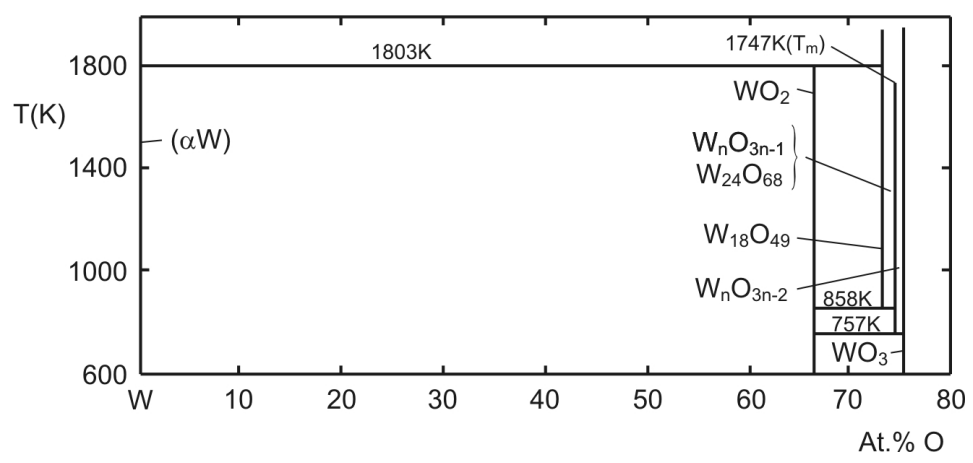

Abbildung 2.3: Phasendiagramm für das System W-O nach [Pred1998]. 


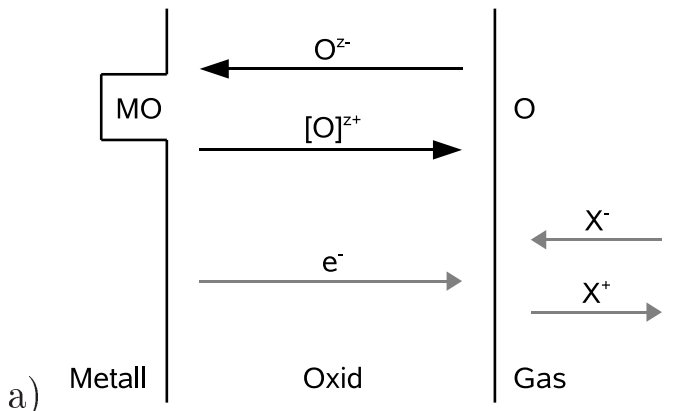

Abbildung 2.4: Teilchenströme während der Oxidationsreaktion mit Lage der Reaktionsfront a) an der Metall-Oxid-Grenzfläche und b) an der Oxidoberfläche.

zeichnet Tieftemperaturoxidation den Vorgang der Oxidation bei Temperaturen die so niedrig sind, dass die thermische Energie nicht zu einer signifikanten Aktivierung der Volumendiffusion ausreicht. Im Anschluss werden Resultate bisheriger Untersuchungen zur Manipulation der natürlichen Tieftemperaturoxidation vorgestellt, wobei insbesondere auf Studien an nanoskaligen Systemen eingegangen wird.

\subsubsection{Teilschritte der Oxidationsreaktion}

Bei der Tieftemperaturoxidation eines Festkörpers durch ein sauerstoffenthaltendes Gas handelt es sich um eine heterogene Reaktion, bei der die Reaktanden bereits nach geringem Fortschreiten der Reaktion durch das Reaktionsprodukt - die entstehende Oxidschicht- räumlich getrennt werden. Die Kinetik der Reaktion wird daher durch eine Vielzahl von Einzelschritten bestimmt, welche im Folgenden betrachtet werden sollen.

Ist lediglich der Sauerstoff in der Oxidschicht mobil, so können folgende Reaktionsschritte auftreten: Antransport des Gases, Physisorption des Gases an der Oxidoberfläche, Chemisorption des Gases an der Oxidoberfläche, Übergang des Sauerstoffs in das Oxid, Diffusion des Sauerstoffs durch das Oxid, Reaktion mit dem Metall an der MetallOxid-Grenzfläche. Ist hingegen allein das Metall in der Oxidschicht mobil, so können die folgenden Reaktionsschritte stattfinden: Übergang eines Metallatoms in das Oxid, Diffusion des Metalls durch das Oxid, Reaktion mit dem physisorbierten oder chemisorbierten Gas an der Oxidoberfläche. Welche der einzelnen Reaktionsschritte dabei im konkreten Fall zu berücksichtigen sind, ist von der Lage der Reaktionsfront abhängig.

Wesentlich für die Kinetik der Reaktion ist jedoch, dass die durch das Oxid diffundierenden Spezies in der Regel nicht neutral, sondern als Metallkationen $\mathrm{M}^{\mathrm{z}+}$ oder Sauerstoffanionen $\mathrm{O}^{\mathrm{z}-}$ beziehungsweise in der Form entsprechender Leerstellen $[\mathrm{M}]^{\mathrm{z}-}$ oder $[\mathrm{O}]^{\mathrm{z}+}$ vorliegen [Fehl1986, From1976, From1998]. Somit sind auch die Reaktionsschritte an der Metall-Oxid-Grenzfläche und an der Oxidoberfläche als Reaktionen geladener Teilchen zu betrachten. In die Reaktionsrate für einen Teilschritt gehen daher als variable Größen nicht nur die Aktivitäten der Reaktanden und der Produkte, sondern auch die durch das lokale elektrische Feld modifizierten Aktivierungsenergien ein.

Weiterhin führt die Diffusion von Metallkationen oder Sauerstoffanionen beziehungsweise der entsprechenden Leerstellen wie in Abb. 2.4 dargestellt stets zu einem Nettotransport positiver Ladung von der Metall-Oxid-Grenzfläche zur Oxidoberfläche. Um 
die Ladungserhaltung sicherzustellen, müssen daher weitere Prozesse, welche einen Nettotransport negativer Ladung an die Oxidoberfläche zur Folge haben, berücksichtigt werden. Dies kann zum einen der Transport von Elektronen $\mathrm{e}^{-}$von der Metall-OxidGrenzfläche zur Oxidoberfläche sein, welcher durch quantenmechanisches Tunneln, thermisch aktivierte Elektronen- oder Lochleitung durch das halbleitende Oxid oder durch einen thermisch aktivierten Sprungmechanismus ("hopping-mechanism") erfolgen kann [From1998]. Zum anderen kommt als Mechanismus für den Nettotransport negativer Ladung an die Oxidoberfläche ein Zustrom negativ geladener Spezies $\mathrm{X}^{-}$aus dem Gasraum oder eine Desorption positiv geladener Spezies $\mathrm{X}^{+}$von der Oxidoberfläche in Frage.

Zur Beschreibung der Kinetik einer Oxidationsreaktion ist es prinzipiell möglich, für alle Reaktionsschritte und alle für den Ladungsausgleich verantwortlichen Prozesse Ratengleichungen zu formulieren und diese zu lösen. Durch ein solches Vorgehen wird jedoch eine große Zahl freier Parameter eingeführt, weshalb die physikalische Aussagekraft der Lösungen im Allgemeinen gering ist. Daher wird häufig das Konzept des geschwindigkeitsbestimmenden Schritts zugrunde gelegt, die Kinetik der gesamten Reaktion wird also auf die des langsamsten Einzelschrittes zurückgeführt, wobei für alle übrigen Teilschritte der thermodynamische Gleichgewichtszustand angenommen wird.

Im Folgenden werden unter Anwendung dieses Konzepts abgeleitete Modelle für die Kinetik der Tieftemperaturoxidation vorgestellt. Da thermisch aktivierte Prozesse bei tiefen Temperaturen nur mit vernachlässigbarer Rate ablaufen, sind dabei insbesondere feldabhängige Prozesse relevant.

\subsubsection{Kinetik der unbeeinflussten Tieftemperaturoxidation}

Eine Vielzahl von Diagrammen zur Oxidationskinetik unterschiedlicher Metalle bei tiefen Temperaturen finden sich in den Monographien von Fehlner [Fehl1986] und Fromm [From1998]. Typischerweise lassen sich dabei in der Auftragung der Oxiddicke gegen eine logarithmische Zeitskala, wie in Abb. 2.5 schematisch dargestellt, drei Regime des Oxidwachstums unterscheiden. Zu Beginn verläuft die Oxidationsreaktion mit sehr hoher Rate, im zweiten Bereich sinkt die Oxidationsrate deutlich ab und im dritten Bereich wird Oxidwachstum schneller als einem logarithmischen Zeitgesetz folgend beobachtet. Das Wachstum im zweiten Regime erfolgt dabei nahezu unabhängig von der Temperatur, während die Kinetik im ersten und dritten Regime temperaturabhängig ist. Bei hinreichend tiefen Temperaturen lässt sich jedoch im dritten Regime im Rahmen der experimentellen Auflösung kein Oxidwachstum mehr nachweisen.

Das im dritten Regime bei höheren Temperaturen erfolgende Wachstum ist auf thermisch aktivierte Diffusion geladener Spezies zurückzuführen, wie sie zuerst von Wagner [Wagn1933] beschrieben wurde. Im ersten und zweiten Regime hingegen verläuft die Oxidationsreaktion auch ohne thermische Aktivierung. Sie führt bei hinreichend hohem Sauerstoffpartialdruck innerhalb von Sekunden zu Oxidschichtdicken von 0,4 nm bei Aluminium [Jeur2002] und von etwa 0,6 nm bei Silizium [Enta2008]. Bei Wolfram stellt sich eine 2 Monolagen atomarem Sauerstoff entsprechende Oxiddicke ein [King1971], bei Eisen und Titan ein 20 bis 30 Monolagen molekularem Sauerstoff äquivalente [Mart1994].

Zur Beschreibung der Oxidationskinetik in den ersten beiden Regimen wurde eine Vielzahl von Modellen vorgeschlagen. Mott [Mott1940] führte den starken Abfall der Oxida- 


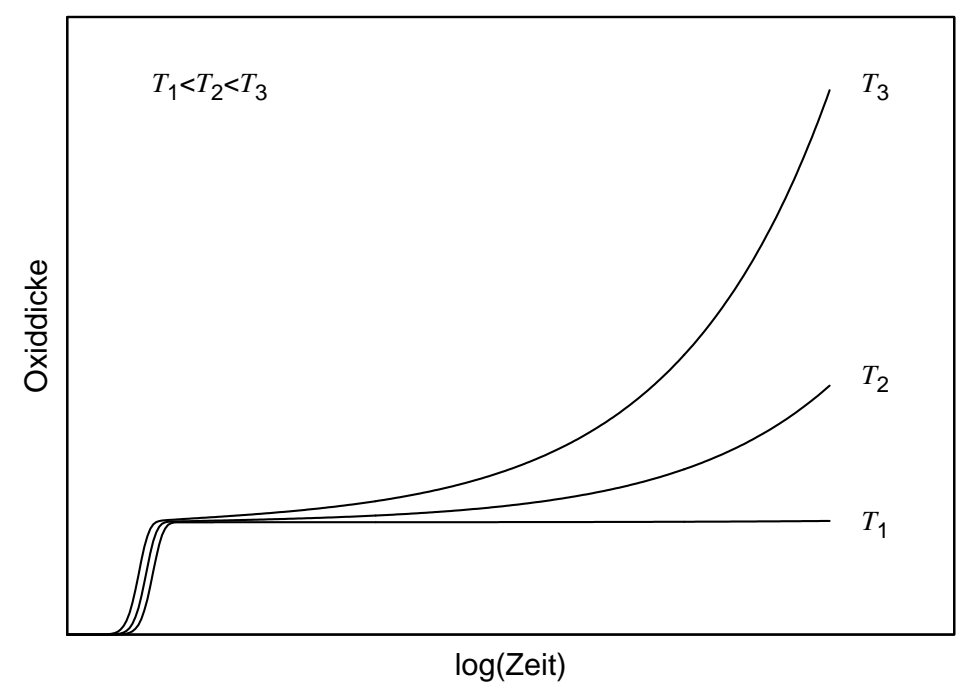

Abbildung 2.5: Schematische Darstellung der typischen Regime für Oxidwachstum bei unterschiedlichen Temperaturen $T_{\mathrm{j}}$.

tionsrate im zweiten Regime auf einen aus Gründen der Ladungsneutralität notwendigen Tunnelvorgang von Elektronen durch die wachsende Oxidschicht zurück und leitete daraus ein logarithmisches Wachstumsgesetz ab. In späteren Arbeiten [Cabr1949, Mott1947] wurde dann erstmals die Diffusion von Ionen durch das Oxid unter Einfluss eines elektrischen Feldes betrachtet. An der Oxidoberfläche chemisorbierter Sauerstoff besitzt demnach Akzeptor-Niveaus welche um den Betrag e $V_{\text {Mott }}$ unterhalb des Fermi-Niveaus des Metalls liegen. Durch Besetzung dieser Niveaus baut sich ein elektrisches Feld $E$ über die Oxidschicht hinweg auf, welches eine zusätzliche Triebkraft für den Diffusionsprozess darstellt. Bezeichnet, wie in Abb. 2.6 dargestellt, $A$ die Aktivierungsenergie für die Diffusion eines Ions der Spezies i im feldfreien Fall, $a$ den halben Abstand zweier benachbarten Minima in der Potentiallandschaft, $\nu$ die Frequenz der thermischen Schwingungen des Ions um seine Ruhelage und $\theta^{\mathrm{v}}, \theta^{\mathrm{r}}$ die Flächendichte mobiler Ionen für Vor- und Rücksprünge, so folgt für die Teilchenstromdichte der Spezies i

$$
j_{\mathrm{i}}=\theta^{\mathrm{v}} \nu \exp \left(\frac{-\left(A-z_{\mathrm{i}} \mathrm{e} a E\right)}{\mathrm{k} T}\right)-\theta^{\mathrm{r}} \nu_{0} \exp \left(\frac{-\left(A+z_{\mathrm{i}} \mathrm{e} a E\right)}{\mathrm{k} T}\right) .
$$

Die Existenz einer Potentialdifferenz zwischen der Metall-Oxid-Grenzfläche und der Oxidoberfläche konnte in verschiedenen Untersuchungen [Bogg1970, Kirk1968, Poll1964] experimentell bestätigt werden. Das Konzept der getriebenen Diffusion liefert somit einen Ansatz zum Verständnis der extrem hohen Oxidationsraten im ersten Regime auch bei tiefen Temperaturen. Durch seine Beschränkung auf einen einzigen geschwindigkeitsbestimmenden Schritt besitzt es jedoch nur begrenzte Gültigkeit.

Eine konsequente Berücksichtigung der Kopplung zwischen ionischen und elektronischen Transportvorgängen findet sich in der Modellierung der Oxidationskinetik durch Fromhold [From1976] und schließlich unter ergänzender Einbeziehung der Reaktionen an der Oxidoberfläche in der detailreichen Darstellung der Kinetik der Tieftemperaturoxidation durch Fromm [From1998]. Die numerische Modellierung experimentell bestimmten Oxidationsverhaltens legt dabei folgendes Verständnis der wesentlichen Mechanismen 
a)

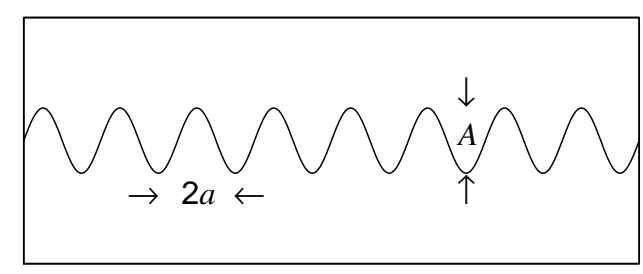

b)

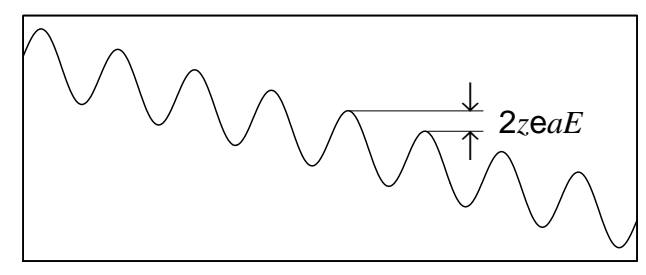

Abbildung 2.6: Potentiallandschaft für ein Ion a) im feldfreien Fall und b) unter dem Einfluss eines elektrischen Feldes $E$.

der Tieftemperaturoxidation nahe: Die Energiedifferenz zwischen dem Fermi-Niveau des Metalls und Akzeptor-Niveaus des an der Oxidoberfläche chemisorbierten Sauerstoffs von etwa $1 \mathrm{eV}$ ist die Ursache für das Entstehen des Mott-Feldes. Dieses erhöht im ersten Regime die Ionentransportrate durch das Oxid um mehrere Größenordnungen sofern die "screening length" im Oxid deutlich größer ist als die Dicke der Oxidschicht. Der anfänglich extrem hohe Tunnelstrom von Elektronen in die Akzeptor-Niveaus des Sauerstoffs ist stark dickenabhängig und wird im zweiten Regime geschwindigkeitsbestimmend für die Oxidationsreaktion. Sofern die thermische Energie zur Anregung alternativer elektronischer Transportmechanismen durch das Oxid ausreicht, kann sich das Oxidwachstum im dritten Regime fortsetzen, andernfalls kommt es im Rahmen der experimentellen Genauigkeit zum Erliegen.

Der starke Einfluss des sich während der Oxidationsreaktion ausbildenden internen elektrischen Feldes auf die Kinetik der Reaktion im ersten Regime legt nahe, den Einfluss externer Felder auf die Kinetik von Oxidationsreaktionen zu untersuchen. Bisherige Arbeiten dazu werden im Folgenden vorgestellt.

\subsubsection{Externe Manipulation der Tieftemperaturoxidation - Planarsysteme}

Vor der Betrachtung der externen Manipulation der Tieftemperaturoxidation soll zunächst die Notwendigkeit der Ladungserhaltung betont werden. Sofern kein Ladungstransport zwischen der Oxidoberfläche und dem Gasraum wie in Abb. 2.4 durch die Spezies X erfolgt, muss der ionische Ladungstransport durch das Oxid während des Oxidwachstums durch elektronischen Ladungstransport kompensiert werden. Unter dem Einfluss eines externen Feldes wird - unabhängig von dessen Orientierung - daher stets ein Transportprozess beschleunigt, während der andere verzögert wird. In Studien zur Manipulation der Tieftemperaturoxidation durch elektrostatische Felder werden aus diesem Grunde die felderzeugenden Ladungen üblicherweise direkt auf die Oxidoberfläche aufgebracht. Somit liegen die elektronischen Randbedingungen wie elektrostatisches Potential oder Ladungsdichte fest, und allein ionische Reaktionen bestimmen die Oxidationskinetik.

In frühen Arbeiten wurde die Oxidationskinetik unter dem Einfluss eines elektrisches Feldes zwischen der zu oxidierenden Probe und einer porösen Platinschicht auf der Oxidoberfläche untersucht. Die Oxidationsrate von Silizium bei $850^{\circ} \mathrm{C}$ lässt sich bei negativem Potential an der Platinelektrode und Feldstärken in der Größenordnung von $10^{6} \mathrm{~V} / \mathrm{m}$ signifikant erhöhen [Jorg1962]. Bei umgekehrter Polarität wird eine Ver- 
ringerung der Oxidationsrate gegenüber dem feldfreien Fall beobachtet, und bei einer kritischen Potentialdifferenz kommt die Oxidationsreaktion schließlich vollständig zum Erliegen. Das beobachtete Verhalten wird auf Diffusion von Sauerstoffionen als für das Oxidwachstum wesentlichen Prozess zurückgeführt. Die kritische Potentialdifferenz entspricht dabei gerade einer Energie, welche identisch mit der Änderung der Gibbsschen freien Energie für die Oxidationsreaktion ist.

Auch bei tiefen Temperaturen wird ein deutlicher Feldeinfluss gefunden. So wird bei Temperaturen von $50{ }^{\circ} \mathrm{C}$ bis $400{ }^{\circ} \mathrm{C}$ unter dem Einfluss eines Feldes von etwa $10^{7} \mathrm{~V} / \mathrm{m}$ eine erhöhte Oxidationsrate von Aluminium unter Sauerstoffatmosphäre gemessen, sofern die Platinelektrode auf negativem Potential gegenüber dem Aluminium liegt [Hunt1970]. Bei umgekehrter Polarität hingegen verringert sich die Oxidationsrate. Dieses Verhalten wird auf Diffusion von Aluminiumionen oder auf eine Reaktion an der Oxidoberfläche als geschwindigkeitsbestimmenden Schritt zurückgeführt. In einer Untersuchung zur Oxidation von Wolfram an Laborluft und Zink an Sauerstoffatmosphäre bei Feldstärken von etwa $10^{7} \mathrm{~V} / \mathrm{m}$ wird die qualitativ gleiche Feldabhängigkeit für Wolfram bei $260{ }^{\circ} \mathrm{C}$ und für Zink bei $390^{\circ} \mathrm{C}$ gefunden [Ande1967]. Bei tieferen Temperaturen hingegen wird eine Abnahme der Oxidationsrate beobachtet, wenn die Platinelektrode auf negativem Potential gegenüber dem Metall liegt. Das Verhalten bei höheren Temperaturen wird auf Sauerstoffdiffusion als geschwindigkeitsbestimmenden Schritt zurückgeführt. Bei der Interpretation des Verhaltens bei den niedrigeren Temperaturen als ladungstransportlimitiert bleibt jedoch die Rolle der Platinelektrode als Quelle für Elektronen unberücksichtigt. In einer Studie zur Oxidation von Nickel unter Sauerstoffatmosphäre und elektrischen Feldern von etwa $10^{7} \mathrm{~V} / \mathrm{m}$, welche analoges Verhalten mit einer Übergangstemperatur von $350{ }^{\circ} \mathrm{C}$ zeigt [Ritc1970], wird schließlich der wesentliche Einfluss der Oxidoberfläche auf die Reaktionsrate erkannt.

Durch direkte Elektronenbestrahlung der Oxidoberfläche konnte in einer Reihe von Untersuchungen der Einfluss von Ladungen auf der Oxidoberfläche auf die Kinetik der Tieftemperaturoxidation untersucht werden, ohne dabei Elektroden auf die Oxidoberfläche aufbringen zu müssen. So verläuft die Oxidation von $\mathrm{Al}(111)$ unter $\mathrm{H}_{2} \mathrm{O}$ Atmosphäre bei $300 \mathrm{~K}$ unter Elektronenbestrahlung mit erhöhter Rate und bis zu größeren Schichtdicken [Ebin1998]. Dies wird auf eine durch die Elektronenbestrahlung verursachte erhöhte Konzentration von Sauerstoffanionen an der Oxidoberfläche zurückgeführt. Das daraus resultierende elektrische Feld von etwa $0,5 \mathrm{~V} / \mathrm{nm}$ treibt dabei dem Cabrera-Mott Modell [Cabr1949, Mott1947] entsprechend den Ionentransport durch die Oxidschicht. Eine erhöhte Oxidationsrate wird auch bei der Oxidation von $\mathrm{Al}(111)$ unter Sauerstoffatmosphäre bei Temperaturen von $90 \mathrm{~K}$ bis $300 \mathrm{~K}$ beobachtet und in ähnlicher Weise interpretiert [Zhuk2002]. Aus der Beobachtung einer erhöhten Oxidationsrate von $\mathrm{Al}(111)$ unter Sauerstoffatomosphäre bei $90 \mathrm{~K}$ auch im Anschluss an eine Elektronenbestrahlung [Popo2002] wird gefolgert, dass ionischer Transport durch die Oxidschicht gemäß dem Cabrera-Mott Modell ursächlich für die erhöhte Oxidationsrate ist. Die entsprechende Feldstärke wird mit etwa $1 \mathrm{~V} / \mathrm{nm}$ angegeben. Die unter Elektronenbestrahlung beobachtete erhöhte Oxidationsrate von Silizium unter Sauerstoffatmosphäre bei $90 \mathrm{~K}$ hingegen wird auf eine elektroneninduzierte Dissoziation von $\mathrm{O}_{2}$ zurückgeführt [Xu1997], da keine Ladung auf der Oxidoberfläche nachgewiesen werden kann [Ohno2005]. 


\subsubsection{Externe Manipulation der Tieftemperaturoxidation - AFM Studien}

Der Einfluss elektrischer Felder auf die Kinetik der Oxidation verbunden mit der Möglichkeit nanometergenauer Strukturierung wurde in zahlreichen Studien mit Atomkraftmikroskopie (AFM) untersucht. Üblicherweise bildet dabei die AFM-Spitze die Kathode mit einer Potentialdifferenz von einigen Volt zur Anode und die Oxidationsreaktion erfolgt bei Raumtemperatur unter Umgebungsatmosphäre mit einer relativen Luftfeuchtigkeit von $10 \%$ bis $95 \%$.

Auf Silizium bildet sich unter diesen Bedingungen bei Feldstärken von einigen V/nm ein bis zu $4 \mathrm{~nm}$ dickes Oxid [Hatt1994], die gemesse Volumenvergrößerung entspricht dabei der bei der Umwandlung von Silizium zu $\mathrm{SiO}_{2}$ erwarteten. Auch Titan kann gezielt in bis zu $15 \mathrm{~nm}$ dickes Titanoxid überführt werden [Dubo2000, Vull1999], jedoch nur bei Potentialdifferenzen oberhalb eines kritischen Wertes von einigen Volt [Vull1999]. Die Oxidation von dotiertem Silizium wird zum Teil ebenfalls erst oberhalb einer von der Dotierung abhängigen kritischen Potentialdifferenz beobachtet [Garc1998, Mori1997, Stié1997, Teus1995]. In anderen Studien hingegen wird keine kritische Potentialdifferenz gefunden [Avou1997]. Mit der Oxidation einhergehend fließt ein Strom zwischen Spitze und Probe [Avou1997, Kura2004], die Ladungsmenge entspricht dabei dem gebildeten Oxidvolumen.

Für die Kinetik der Oxidationsreaktion, ermittelt aus der Abhängigkeit der Oxiddicke $h(t)$ von der Zeit $t$, werden unterschiedliche Zeitgesetze gefunden. Logarithmisches Wachstum $h \propto \ln \left(t / t_{0}\right)$ wird bei der Oxidation von Silizium [Avou1997] und Titan [Vull1999] beobachtet. Für die gleichen Materialien werden jedoch auch die Potenzgesetze $h \propto t^{0,23}$ bei unterschiedlich dotiertem Silizium [Teus1995] und $h \propto t^{0,2}$ bei Titan [Dubo2000] gefunden.

Der kritische Einfluss der relativen Luftfeuchtigkeit auf die Oxidationsreaktion zeigt sich in der Ausbildung größeren Oxidstrukturen bei höherer Luftfeuchtigkeit [Avou1997]. Snow und Mitarbeiter [Snow2000] deuten die stark unterschiedlichen Oxidationsraten von Silizium bei Variation von Potentialdifferenz, Pulsdauer und Oberflächenbeschaffenheit durch einen Wechsel des Aggregatzustands des $\mathrm{H}_{2} \mathrm{O}$. Als geschwindigkeitsbestimmender Schritt für die Oxidationsreaktion wird die Produktionsrate von Sauerstoffanionen angesehen, welche von der lokalen Dichte der $\mathrm{H}_{2} \mathrm{O}$-Moleküle abhängt. Der Anstieg der Oxidationsrate um mehrere Größenordnungen bei hohen Potentialdifferenzen und langer Pulsdauer wird auf die Ausbildung eines Wassermeniskus zwischen Spitze und Probe zurückgeführt, auf dessen Existenz Kraft-Weg Messungen [Avou1997], Experimente mit variabler Potentialdifferenz und Pulsdauer [Garc1998] sowie der Vergleich von Feldberechungen mit der Oxidmorphologie [Avou1997, Mori1997] hinweisen. Auch eine numerische Simultation zur AFM induzierten Oxidation [Djur2007] zeigt die Ausbildung eines Wassermeniskus bei Berücksichtigung der Wechselwirkung zwischen dem permanenten Dipolmoment des $\mathrm{H}_{2} \mathrm{O}$ und dem elektrichen Feld zwischen Spitze und Probe.

Neben der wesentlichen Rolle des elektrischen Feldes und des $\mathrm{H}_{2} \mathrm{O}$ werden in einigen Studien noch mechanische Spannungen [Avou1997] und Raumladungseffekte [Daga1998, Djur2007, Dubo2000, Teus1995] zur Erklärung der Wachstumsraten herangezogen. Der 
Einfluss der einzelnen Faktoren auf die Kinetik des Oxidwachstums ist wegen der Komplexität des Problems jedoch noch nicht geklärt [Xie2006]. Insbesondere der Zustand des $\mathrm{H}_{2} \mathrm{O}$ auf der Oxidoberfläche und die damit verbundene Frage, ob es sich bei der AFM induzierten Oxidation um eine Reaktion zwischen einem Festkörper und einem Gas oder aber um eine elektrochemische Reaktion zwischen einem Festkörper und einer Flüssigkeit [Garc2006] handelt, sind dabei von Interesse.

Die bisherigen Untersuchungen zur Manipulation der Tieftemperaturoxidation zeigen, dass der natürliche Oxidationsprozess an der Oberfläche von Metallen und Halbleitern durch äußere elektrische Felder erheblich beeinflusst werden kann. Ein Verständnis der zugrunde liegenden Mechanismen wird jedoch dadurch erschwert, dass durch das Aufbringen von Elektroden oder Elektronenbestrahlung stets auch die Aktivitäten der Spezies an der Oxidoberfläche verändert werden. Daher sind Untersuchungen zur Oxidation an nanoskaligen Systemen von Interesse, denn an ihrer Oberfläche können, bedingt durch deren starke Krümmung, allein durch eine Potentialdifferenz zu einer makroskopischen Gegenelektrode Feldstärken von einigen $10 \mathrm{~V} / \mathrm{nm}$ realisiert werden. 


\section{Methoden}

Den Schwerpunkt dieses Kapitels bildet die Vorstellung der im Rahmen dieser Arbeit verwendeten experimentellen Methoden. Neben der Probenpräparation und dem experimentellen Aufbau wird dabei insbesondere die schrittweise Durchführung der Oxidationsreaktion beschrieben, die wegen der Verwendung von ex situ Charakterisierungsmethoden notwendig ist. Abschließend wird das Vorgehen bei der Berechnung des elektrischen Feldes mit der Finite-Elemente-Methode erläutert.

\subsection{Probenpräparation}

In diesem Abschnitt wird die Präparation von nanoskaligen Spitzen aus Wolfram, Aluminium und Silizium, deren Oxidationsverhalten im Folgenden untersucht werden soll, vorgestellt. Zwingend notwendig ist dabei, dass die Spitzen und gegebenenfalls auch der Experimentator selbst während der Handhabung stets geerdet sind. Durch Erdfreiheit können ansonsten Potentialdifferenzen auftreten, welche unkontrollierte Reaktionen der nanoskaligen Spitzen mit der Umgebungsatmosphäre induzieren.

\subsubsection{Elektrochemische Präparation der W- und Al-Proben}

Die nanoskaligen Spitzen aus Wolfram und Aluminium werden durch elektrochemisches Ätzen entsprechender Drähte (W: Reinheit 99,9+\%, Durchmesser $70 \mu \mathrm{m}$; Al: Reinheit 99,99+\%, Durchmesser $125 \mu \mathrm{m}$ ) hergestellt. Zur Verbesserung der Handhabung werden dabei einzelne Stücke des jeweiligen Drahtes derart in $15 \mathrm{~mm}$ lange Kupferhohlzylinder eingeklemmt, dass sie etwa $12 \mathrm{~mm}$ hervorstehen. ${ }^{1}$ Der Kupferhohlzylinder zusammen mit dem eingeklemmten Draht wird im Folgenden als Probe bezeichnet.

Für Wolfram wird als Elektrolyt $\mathrm{NaOH}$ mit einer Konzentration von $2 \mathrm{~mol} / 1$ verwendet. Geätzt wird bei $50 \mathrm{~Hz}$ Wechselspannung mit einer Potentialdifferenz von $7 \mathrm{~V}$ bis $3 \mathrm{~V}$ zwischen der Spitze und einer flächenhaften Gegenelektrode aus Platin. Als Elektrolyt für Aluminium wird eine Mischung aus einem Teil Salpetersäure (65\%) und zwei Teilen Methanol verwendet. Zwischen der Spitze als Anode und einer ringförmigen Platinkathode wird eine Rechteckspannung von $7 \mathrm{~V}$ mit einer Pulsdauer zwischen $6 \mathrm{~ms}$ und $240 \mathrm{~ms}$ angelegt. Die Periodendauer beträgt 1,2 s. Der Ätzvorgang wird unter einem Lichtmikroskop beobachtet, während die oben angegebenen Potentialzustände für eine abnehmende Dauer von einigen Sekunden bis zu einigen Millisekunden angelegt werden. Sobald sich die sichtbare Länge der Spitzen sprunghaft verringert hat, wird der Ätzprozess beendet. In dieser Situation besitzen die Spitzen im Apexbereich üblicherweise einen Krüm-

\footnotetext{
${ }^{1}$ Der Außendurchmesser der Kupferhohlzylinder beträgt 1,5 mm, der Innendurchmesser 0,6 mm.
} 
a)

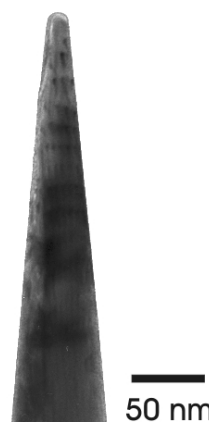

b)

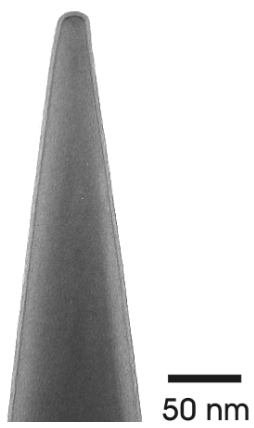

c)

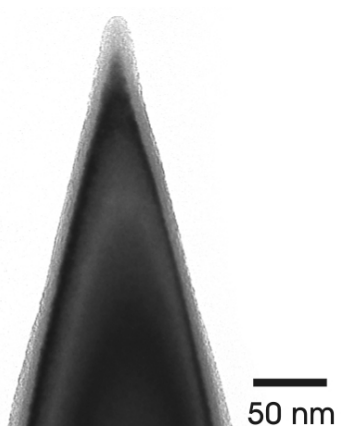

Abbildung 3.1: TEM-Bilder der für die Oxidationsuntersuchungen präparierten Spitzen aus a) Wolfram, b) Aluminium und c) Silizium. Bei Wolfram ist mit konventioneller TEM keine Oxidschicht auflösbar. Die Schicht auf der Al-Spitze entsteht während des elektrochemischen Ätzens. Die amorphe Oberfläche beim Silizium resultiert aus der Bestrahlung mit Ga-Ionen im FIB.

mungsradius von weniger als $10 \mathrm{~nm}$. Die Spitzen werden aus dem Elektrolyten entfernt und durch Eintauchen in Methanol gereinigt.

Nach dem elektrolytischen Ätzen werden die Proben in ein Feldionenmikroskop (FIM) mit einem Restgasdruck von weniger als $10^{-9}$ mbar eingebaut und auf eine Temperatur von $60 \mathrm{~K}$ gekühlt. Durch Anlegen eines positiven Potentials an die Probe gegenüber einer einige Zentimeter entfernten, ringförmigen Gegenelektrode auf Erdpotential kann die Spitzenoberfläche mittels eines geeigneten Bildgases (W: $5 \cdot 10^{-5}$ mbar Helium, Al: $5 \cdot 10^{-5}$ mbar Neon) derart stark vergrößert abgebildet werden, dass einzelne Atome aufgelöst werden können. Bei hinreichend hohen Feldstärken an der Spitze (W: $57 \mathrm{~V} / \mathrm{nm}$, $\mathrm{Al}$ : etwa $20 \mathrm{~V} / \mathrm{nm}$ [Mill1996]) werden darüberhinaus Oberflächenatome von der Spitze abgetragen. Dadurch stellt sich eine gleichmäßige Verrundung der Spitze im Apexbereich ein, welche wegen der simultanen Abbildung durch das Bildgas direkt beobachtet werden kann. Durch Anlegen einer Potentialdifferenz zwischen Probe und Gegenelektrode von $5 \mathrm{kV}$ bei Wolfram und von $2 \mathrm{kV}$ bei Aluminium erhält man somit Spitzen mit einer glatten Kontur und Krümmungsradien von etwa $10 \mathrm{~nm}$ bis $15 \mathrm{~nm}$ im Apexbereich.

Nachdem die Proben wieder Umgebungstemperatur angenommen haben, werden sie aus dem Feldionenmikroskop ausgebaut und mittels Transmissionselektronenmikroskopie (TEM, siehe 3.4.1) charakterisiert. Da sich die Proben zusammen mit dem Probenhalter des Elektronenmikroskopes um $90^{\circ}$ um die Längsachse der Probe drehen lassen, kann sichergestellt werden, dass die Proben einen kreisförmigen Querschnitt besitzen. Dabei werden Abweichungen zwischen maximalem Durchmesser und minimalem Durchmesser von $15 \%$ toleriert. Weiterhin wird durch die transmissionselektronenmikroskopische Untersuchung sichergestellt, dass neben dem Apexbereich auch der durch das elektrochemische Ätzen definierte Schaft der Spitzen hinreichend glatt ist. Lediglich Spitzen, welche diesen beiden Anforderungen genügen, werden für die Oxidationsuntersuchungen verwendet. Typische Beispiele zeigt Abb. 3.1.

\subsubsection{Präparation der Si-Proben mit FIB}

Die Si-Spitzen werden mittels eines fokussierten Ionenstrahls (Focused-Ion-Beam, FIB) aus mit Bor dotiertem einkristallinem Silizium (spezifischer Widerstand $8 \mathrm{k} \Omega \mathrm{m}$ ) her- 


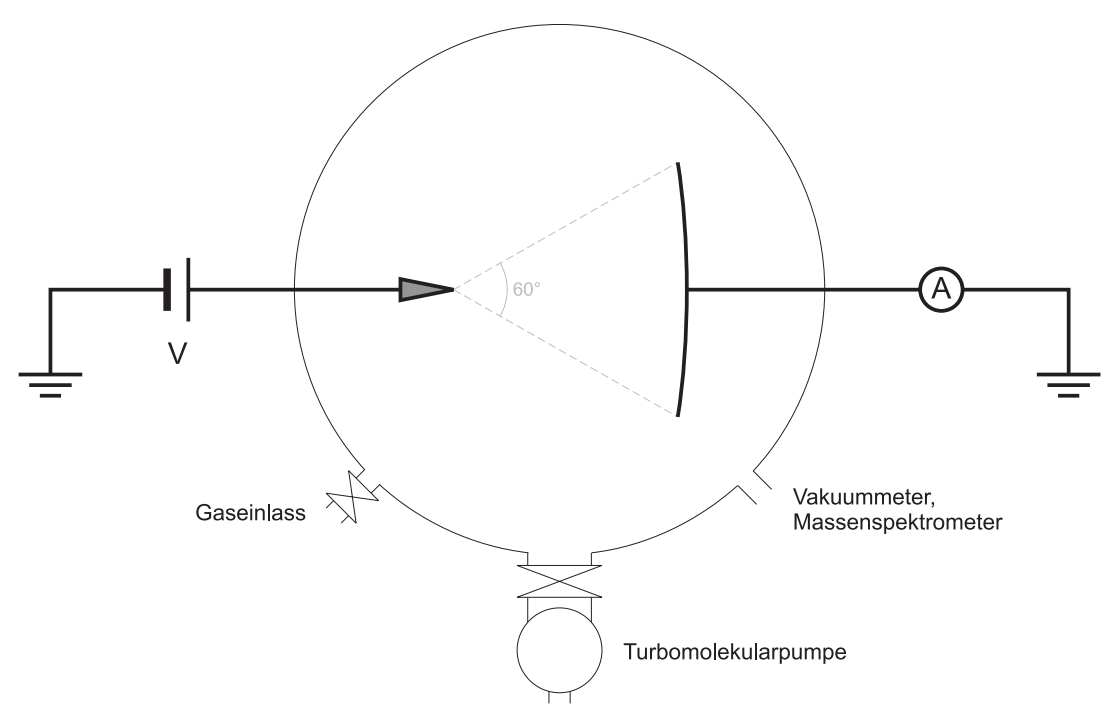

Abbildung 3.2: Schematische Darstellung der Reaktionskammer, die zur Untersuchung des Oxidationsverhaltens nanoskaliger Spitzen aufgebaut wurde.

auspräpariert. Die Einzelschritte des Präparationsvorganges erfolgen dabei gemäß der in [Mill2005] beschriebenen "lift-out" Methode. Die Probenerosion erfolgt durch GaIonen, es wird eine Trägerspitze aus Wolfram verwendet und die mechanische Verbindung zwischen Trägerspitze und Silizium wird durch ioneninduzierte Abscheidung eines $\left(\mathrm{CH}_{3} \mathrm{C}_{5} \mathrm{H}_{4}\right) \mathrm{Pt}\left(\mathrm{CH}_{3}\right)_{3}$ Precursors hergestellt. Da mit der FIB Präparation eine signifikante Strahlenschädigung des Siliziums einhergeht, weisen die oberflächennahen Bereiche der Si-Spitzen eine amorphe Struktur auf [Gian2005]. Bei einer Energie der Ga-Ionen von $5 \mathrm{keV}$ beträgt die Dicke dieser amorphen Schicht etwa $10 \mathrm{~nm}$ (siehe Abb. 3.1).

Durch die Präparation der Si-Spitzen mit dem FIB ist der Apexbereich der Spitzen bereits gleichmäßig verrundet, daher werden die Si-Spitzen nicht im Feldionenmikroskop betrachtet. Sie werden nach dem FIB Prozess wie in 3.1.1 beschrieben im TEM charakterisiert und dann für die Oxidationsuntersuchungen verwendet.

\subsection{Experimenteller Aufbau}

Zur Untersuchung des Oxidationsverhaltens nanoskaliger Spitzen wurde im Rahmen der hier vorgestellten Studie ein Vakuumrezipient aufgebaut und in eine bestehende UHVAnlage integriert. Die Anlage ermöglicht es, Proben über eine Schleuse in eine Austauschkammer $\left(p<2 \cdot 10^{-8} \mathrm{mbar}\right)$ einzubringen. Von dort lassen sie sich einzeln in die neu aufgebaute Reaktionskammer transferieren, in der die Oxidationsreaktion stattfindet. Eine schematische Darstellung dieser Kammer zeigt Abb. 3.2.

In der Reaktionskammer befindet sich die Probe in einem Abstand von $50 \mathrm{~mm}$ vor einer Gegenelektrode aus Kupfer, deren Durchmesser $60 \mathrm{~mm}$ beträgt. An die Probe kann ein positives Potential $V<2000 \mathrm{~V}$ gegenüber Erdpotential angelegt werden. Die Gegenelektrode liegt auf Erdpotential, wobei ein zwischen Gegenelektrode und Erde fließender Strom mit einem Amperemeter (KeITHLey 617) gemessen werden kann. Die zeitabhängige Erfassung des Stromes erfolgt computergesteuert mit einer Zeitauflösung von 


\begin{tabular}{ccccccc} 
Total & $\mathrm{H}_{2} \mathrm{O}$ & $\mathrm{O}_{2}$ & $\mathrm{~N}_{2} / \mathrm{CO}$ & $\mathrm{CO}_{2}$ & $\mathrm{H}_{2}$ & $\mathrm{C}_{\mathrm{m}} \mathrm{H}_{\mathrm{n}}$ \\
\hline $1,0 \cdot 10^{-7}$ & $9,7 \cdot 10^{-8}$ & $7,0 \cdot 10^{-10}$ & $1,9 \cdot 10^{-9}$ & $3,3 \cdot 10^{-10}$ & $8,4 \cdot 10^{-10}$ & $3,7 \cdot 10^{-10}$ \\
$1,0 \cdot 10^{-6}$ & $9,7 \cdot 10^{-7}$ & $6,7 \cdot 10^{-9}$ & $8,5 \cdot 10^{-9}$ & $1,9 \cdot 10^{-9}$ & $5,6 \cdot 10^{-9}$ & $1,3 \cdot 10^{-9}$ \\
$1,0 \cdot 10^{-5}$ & $9,8 \cdot 10^{-6}$ & $7,2 \cdot 10^{-8}$ & $5,1 \cdot 10^{-8}$ & $1,9 \cdot 10^{-8}$ & $3,0 \cdot 10^{-8}$ & $1,0 \cdot 10^{-8}$
\end{tabular}

Tabelle 3.1: Mittels Massenspektrometrie bestimmte Partialdrücke der Gasatmosphäre in der Reaktionskammer bei Gasdurchfluss von $\mathrm{H}_{2} \mathrm{O}$ (siehe 3.3), alle Angaben in mbar. Für Totaldrücke von $10^{-7}$ mbar, $10^{-6}$ mbar und $10^{-5}$ mbar sind die Partialdrücke der sauerstoff- und wasserstoffenthaltenden Gase angegeben.

$350 \mathrm{~ms}$, der Rauschpegel der Strommessung liegt bei $2 \cdot 10^{-14} \mathrm{~A}$.

Über ein regelbares Ventil kann Sauerstoff (Reinheit >99,998 Vol.\%), Laborluft oder $\mathrm{H}_{2} \mathrm{O}$ (Dampf über demineralisiertem Wasser in einem Reservoir aus Quarzglas) in die Reaktionskammer eingelassen werden. Die Zusammensetzung der $\mathrm{H}_{2} \mathrm{O}$-Atmosphäre lässt sich dabei mit einem Massenspektrometer bestimmen. Bei Totaldrücken von $10^{-7}$ mbar bis $10^{-5}$ mbar unter Gasdurchfluss (siehe 3.3) ergeben sich so die in Tab. 3.1 zusammengefassten Partialdrücke. Der Partialdruck von Sauerstoff beträgt demnach auch bei niedrigen Drücken weniger als $1 \%$ des Totaldruckes.

Die Druckmessung in der Reaktionskammer erfolgt für $p<5 \cdot 10^{-4} \mathrm{mbar}$ mit einem Kaltkathoden-Ionisationsvakuummeter, für $5 \cdot 10^{-4}$ mbar $\leq p<1$ mbar mit einem Wärmeleitungs-Vakuummeter und für $p \geq 1$ mbar mit einem kapazitiven Vakuummeter. Der Restgasdruck in der Kammer beträgt weniger als $10^{-7}$ mbar.

\subsection{Durchführung der Oxidationsreaktion und in situ Charakterisierung}

Sämtliche Untersuchungen zur Oxidation nanoskaliger Spitzen wurden bei einer Spitzentemperatur von $296 \mathrm{~K}$ durchgeführt. Vor Beginn der Oxidationsexperimente wird die Zuleitung für das sauerstoffenthaltende Gas gespült, indem das Gaseinlassventil für mindestens $30 \mathrm{~s}$ soweit geöffnet wird, dass sich bei voller Pumpleistung ein Druck von $5 \cdot 10^{-3}$ mbar in der Kammer einstellt. Nachdem der Druck in der Kammer wieder auf unter $10^{-5}$ mbar abgesunken ist, wird eine Probe von der Austauschkammer in die Reaktionskammer transferiert. Für Experimente bei einem Druck von $p \geq 10^{-1}$ mbar wird das Ventil zwischen der Turbomolekularpumpe und dem Rezipienten geschlossen und über das Gaseinlassventil der gewünschte Druck eingestellt. Für Experimente bei geringerem Druck wird das Ventil zwischen der Turbomolekularpumpe und dem Rezipienten hingegen nicht vollständig geschlossen. Die Oxidationsreaktion erfolgt stattdessen bei einem stetigen Gasdurchfluss.

Nachdem sich der Druck in der Reaktionskammer auf den gewünschten Wert stabilisiert hat, werden das Kaltkathoden-Ionisationsvakuummeter und das WärmeleitungsVakuummeter ausgeschaltet. Für eine definierte Zeit $t_{1}$ wird nun die Spannung $V_{1}$ an die Spitze angelegt. Durch die Strommessung kann dabei der Verlauf der Oxidationsreaktion in situ verfolgt werden. Nach Ablauf der Zeit $t_{1}$ wird das Gaseinlassventil geschlossen und die ausgeschalteten Vakuummeter werden wieder in Betrieb gesetzt. Nachdem der 
a)

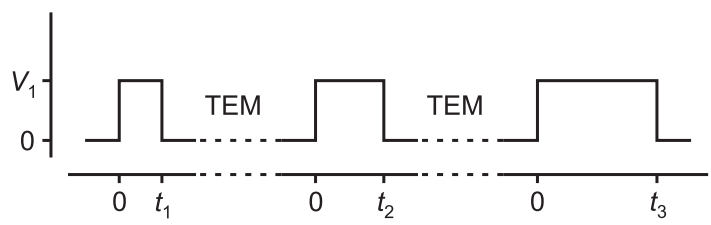

b)

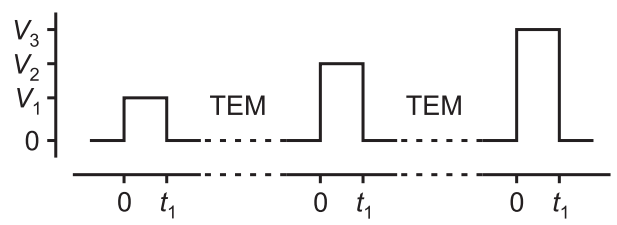

Abbildung 3.3: Schematische Darstellung der Zeitabhängigkeit des Spannungsverlaufes für a) die Untersuchung der Kinetik der Oxidationsreaktion und b) die Untersuchung der Spannungsabhängigkeit der Oxidationsreaktion.

Druck in der Kammer auf unter $10^{-5}$ mbar abgesunken ist, wird die Probe zurück in die Austauschkammer transferiert. Im Anschluss wird die Probe im TEM charakterisiert.

Zur Untersuchung der Kinetik des Oxidationsverhaltens nanoskaliger Spitzen wird dieselbe Probe erneut in die Reaktionskammer eingebaut und bei demselben Druck wird wiederum die Spannung $V_{1}$ für die Zeit $t_{2}$ angelegt. Im Anschluss folgt wieder eine Charakterisierung im TEM. Durch mehrmalige Wiederholung dieser Schritte ergibt sich schließlich die Gesamtzeit für die Oxidationsreaktion zu $t=\sum t_{\mathrm{j}}$, wobei durch die Charakterisierung im TEM für alle Zeiten $t_{\mathrm{m}, \mathrm{n}}=\sum_{\mathrm{j}=1}^{\mathrm{n}} t_{\mathrm{j}}$ die Morphologie der Spitzen bekannt ist.

Des Weiteren wird der Einfluss der an der Probe anliegenden Spannung auf die Oxidationsreaktion untersucht. Dazu werden die Proben nach dem ersten Oxidationsschritt erneut in die Reaktionskammer eingebracht, bei demselben Druck wie beim ersten Schritt wird nun jedoch während der Zeit $t_{1}$ eine höhere Spannung $V_{2}$ angelegt. Im Anschluss folgt wieder eine Charakterisierung im TEM. Somit liegt jede Spannung $V_{\mathrm{j}}$ für die Zeit $t_{1}$ an der Spitze an. Eine schematische Darstellung des zeitabhängigen Spannungsverlaufes für beide Versuchsreihen zeigt Abb. 3.3.

\subsection{Ex situ Charakterisierungsmethoden}

Zur ex situ Charakterisierung der nanoskaligen Spitzen werden konventionelle Transmissionselektronenmikroskopie und Laser-unterstützte Atomsondentomographie eingesetzt. Die Spezifika beider Methoden werden in diesem Abschnitt beschrieben.

\subsubsection{Transmissionselektronenmikroskopie}

Die Charakterisierung der Morphologie und Struktur der nanoskaligen Spitzen erfolgt mit einem Philips CM 12 bei $120 \mathrm{kV}$ Beschleunigungsspannung. Aufnahmen des Realraums mit bis zu 200.000-facher Vergrößerung werden mit elektronenempfindlichem Photomaterial angefertigt, wodurch eine Aufösung von $0,5 \mathrm{~nm}$ erreicht wird. Zur Bestimmung der Struktur der auf den nanoskaligen Spitzen gebildeten Oxide werden Feinbereichsbeugung und Feinstrahlbeugung verwendet. Die Ermittlung der Beugungskonstante erfolgt dabei durch Auswertung des Beugungsbildes der in der Regel einkristallinen Spitze.

Eine chemische Charakterisierung der Oxidschichten erfolgt mittels energiedispersiver Röntgenspektrometrie (EDX) mit einem PHILIPS EM 420 ST mit $120 \mathrm{kV}$ Beschleuni- 
gungsspannung. Durch Wahl eines Sondendurchmessers von einigen $10 \mathrm{~nm}$ können dabei die chemischen Informationen der Oxidschicht und der Spitze separiert werden.

\subsubsection{Atomsondentomographie}

Zur quantitativen chemischen Charakterisierung der Oxidschichten werden die nanoskaligen Spitzen mittels Laser-unterstützter Atomsondentomographie untersucht. ${ }^{2}$ Da die Proben bereits eine für die Analyse mit Atomsondentomographie geeignete Form besitzen, sind dazu keine weiteren Präparationsschritte notwendig.

Die Messungen werden bei einer Probentemperatur von $40 \mathrm{~K}$ und einem Restgasdruck von weniger als $10^{-9}$ mbar durchgeführt. Zur Anregung der Probe kommt ein gepulster Laser mit einer Wellenlänge von $355 \mathrm{~nm}$ zum Einsatz, die Pulsfrequenz beträgt $1 \mathrm{kHz}$, die Pulsdauer weniger als 500 ps und die Energie pro Puls etwa $15 \mu \mathrm{J}$ bei einem Strahldurchmesser von etwa $1 \mathrm{~mm}$ am Ort der Probe.

In der vorliegenden Konfiguration der Atomsonde können durch Flugzeitmessung Ionen mit einem Massen-Ladungs-Verhältnis von bis zu $250 \mathrm{u} / \mathrm{e}$ identifiziert werden. Da bei der Laser-unterstützten Atomsondentomographie von Oxiden ein erheblicher Anteil der Ionen nicht atomar sondern molekular vorliegt, erfolgt die Zuordnung der Peaks im Massenspektrum durch einen Vergleich mit allen möglichen Molekülzusammensetzungen $\mathrm{M}_{\mathrm{x}} \mathrm{O}_{\mathrm{y}}$. Zur Rekonstruktion der dreidimensionalen Atomverteilung werden den einzelnen Molekülen dann entsprechend unterschiedliche Volumina zugeordnet.

\subsection{Feldberechnungen mit der Finite-Elemente-Methode}

Zur Berechnung des elektrischen Feldes in der Umgebung der nanoskaligen Spitzen wird das auf der Finite-Elemente-Methode (FEM) basierende Programm COMSOL MultiPHYSICS verwendet. Grundlage für die Berechnungen ist dabei jeweils die mittels TEM bestimmte Morphologie von Spitze und Oxidschicht.

Sämtliche Berechnungen erfolgen unter der Voraussetzung, dass sich keine freien Ladungsträger in den Oxidschichten auf den nanoskaligen Spitzen befinden. Der Betrag der elektrischen Feldstärke $E=|\nabla \Phi|$ ergibt sich dann durch Lösung der Poissongleichung $\nabla \cdot\left(\varepsilon_{0} \varepsilon_{\mathrm{r}} \nabla \Phi\right)=0$ unter Dirichlet Randbedingungen (gegebenes elektrostatisches Potential $\Phi)$ für Spitze und Gegenelektrode und von Neumann Randbedingung (verschwindende Normalkomponente der dielektrischen Verschiebung $D=\varepsilon_{0} \varepsilon_{\mathrm{r}} E$ ) für die Fläche zwischen den Elektroden. Dabei wird für das Oxid eine isotrope Permittivität mit der Permittivitätszahl $\varepsilon_{\mathrm{r}}$ und für den Gasraum die Permittivität des Vakuums angenommen. Wegen der Symmetrie der Proben und des experimentellen Aufbaus wurden alle Berechnungen in Zylinderkoordinaten $(z, r, \varphi)$ durchgeführt. Somit ergibt sich eine weitere Randbedingung für $r=0$ aus der Forderung nach Kontinuität.

\footnotetext{
${ }^{2}$ Die Untersuchungen mit Laser-unterstützter Atomsondentomographie werden in der Arbeitsgruppe von Prof. Dr. G. Schmitz am Institut für Materialphysik der Westfälischen Wilhelms-Universität Münster durchgeführt. Details zu der eingesetzten Atomsonde finden sich in [Sten2007].
} 

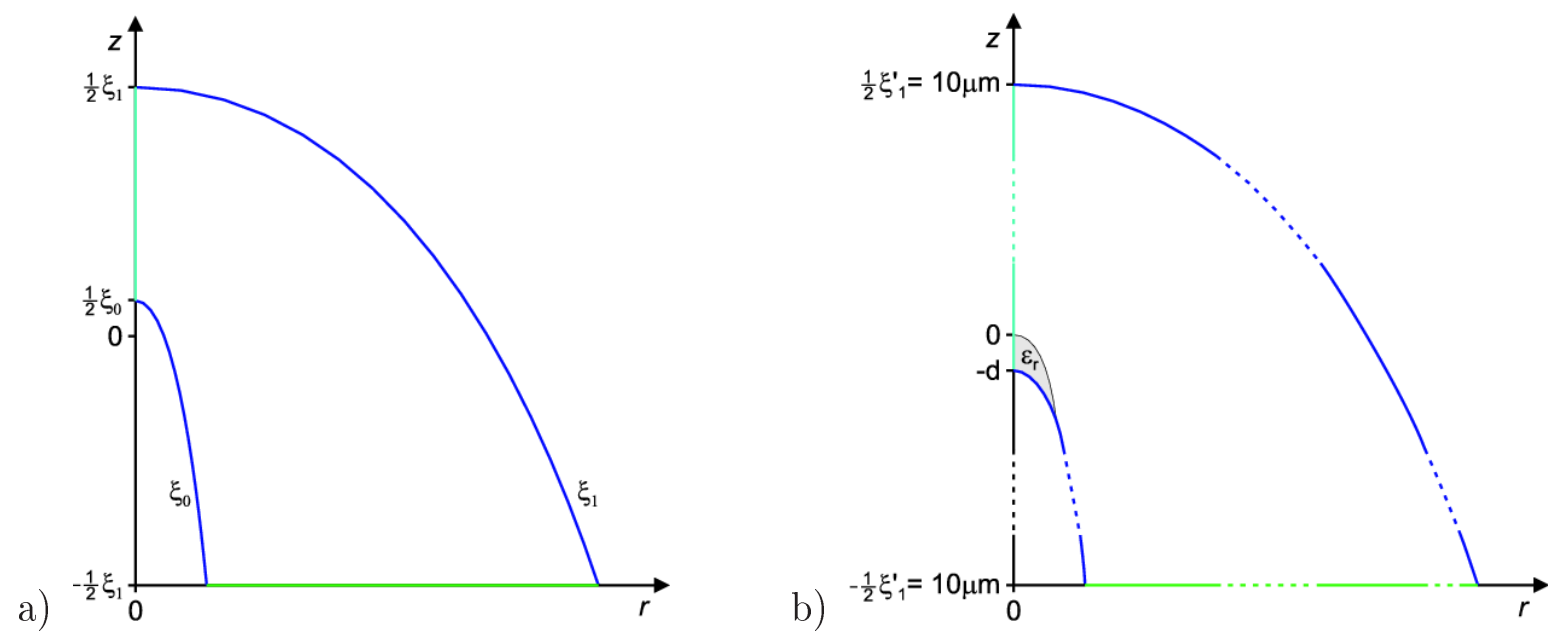

\begin{abstract}
Abbildung 3.4: a) Schematische Darstellung der Elektroden im parabolischen Modell. b) Schematische Darstellung der Geometrie, welche der Berechnung des elektrischen Feldes in der Umgebung der nanoskaligen Spitzen zugrunde liegt. Die Kontur der Spitze samt Oxidschicht wird direkt aus TEMAufnahmen übertragen. Das Potential der Gegenelektrode liegt wie im Text dargestellt bei $0,552 \mathrm{~V}$. In beiden Teilabbildungen bezeichnet | Dirichlet-Randbedingungen, | die von Neumann-Randbedingung und $\mid$ die Randbedingung $r=0$.
\end{abstract}

Da der Krümmungsradius der nanoskaligen Spitzen um bis zu sieben Größenordnungen geringer ist als der Abstand zwischen Spitze und der makroskopischen Gegenelektrode, ist bei der Berechnung durch geeignete Wahl der Parameter sicherzustellen, dass in der unmittelbaren Umgebung der Spitze eine hinreichend exakte Lösung gewonnen wird. $\mathrm{Zu}$ diesem Zweck werden Spitze und Gegenelektrode zunächst durch zwei konfokale Rotationsparaboloide modelliert, welche in Zylinderkoordinaten durch $(z, r, \varphi)=\left(\frac{1}{2}(\eta-\xi), \sqrt{\xi \eta}, \varphi\right)$ für jeweils festes $\xi$ gegeben sind [Mill1996, S. 43]. Ein konstantes $\xi$ beschreibt folglich eine Äquipotentialfläche, ein konstantes $\eta$ für ein festes $\varphi$ eine Feldlinie. Wie in Abb. 3.4 a) dargestellt, werden die Oberfläche der Spitze durch $\xi_{0}$ und die Gegenelektrode durch $\xi_{1}$ repräsentiert. $\xi_{0}$ ist dabei identisch mit dem Krümmungsradius der Spitze. Der Abstand zwischen Spitze und Gegenelektrode auf der Symmetrieachse beträgt $\frac{1}{2}\left(\xi_{1}-\xi_{0}\right)$. Für diese Konfiguration wird mit der FiniteElemente-Methode die Feldstärke $E$ am Apex der Spitze für unterschiedliche Verhältnisse $\xi_{1} / \xi_{0}$ bestimmt. Dabei werden die in Abb. 3.4 a) angegebenen Randbedingungen berücksichtigt. Der Vergleich mit der analytischen Lösung für die Feldstärke am Apex $E\left(\xi_{0}, 0\right)=-2|V|\left(\xi_{0} \ln \left(\xi_{0} / \xi_{1}\right)\right)^{-1}$ [Nowa2006] zeigt, dass für $\xi_{1}=1000 \xi_{0}$ die endliche Ausdehnung der Elektroden in der Berechnung mit der Finite-Elemente-Methode keinen signifikanten Einfluss mehr auf das Feld am Apex der Spitze hat. Gleichzeitig erfolgt die numerische Bestimmung der Feldstärke noch hinreichend schnell. Die Größe der Finiten Elemente in unmittelbarer Umgebung der Spitze beträgt dabei 0,5 nm und es werden quadratische Ansatzfunktionen verwendet.

Die Berechnung des elektrischen Feldes für die in Abb. 3.2 skizzierte Geometrie erfolgt in zwei Schritten: Zunächst wird eine analytische Nährung für den Potentialverlauf betrachtet. Mit der Lösung für das Potential auf einer Zwischenebene wird dann im zweiten Schritt das elektrische Feld in der Umgebung der Spitze mit der Finite-ElementeMethode bestimmt. Für dieses Vorgehen werden Spitze und Gegenelektrode zunächst 
durch Rotationsparaboloide angenähert. Dabei wird ein typischer Krümmungsradius der Spitze am Apex von $\xi_{0}=20 \mathrm{~nm}$ angesetzt, und aus dem axialen Abstand zwischen Spitze und Gegenelektrode von $50 \mathrm{~mm}$ folgt $\xi_{1}=100 \mathrm{~mm}$. Mit der analytischen Lösung für das elektrostatische Potential $\Phi$ im parabolischen Modell $\Phi(\xi)=V \ln \left(\xi / \xi_{1}\right) / \ln \left(\xi_{0} / \xi_{1}\right)$ [Mill1996, S. 46] ergibt sich das Potential für $\xi_{1}^{\prime}=1000 \xi_{0} \mathrm{zu} \Phi\left(\xi_{1}^{\prime}\right)=0,552 \mathrm{~V}$. Die durch $\xi_{1}^{\prime}$ gegebene Fläche konstanten Potentials repräsentiert die Dirichlet-Randbedingung der Gegenelektrode. Die Zuweisung der übrigen Randbedingungen geschieht wie zu Beginn dieses Abschnittes beschrieben. Die Kontur der nanoskaligen Spitze samt Oxidschicht wird gemäß der TEM-Aufnahmen modelliert, so dass sich eine Geometrie wie in Abb. 3.4 b) ergibt. Die Berechnung des elektrischen Feldes erfolgt dann mit den bereits genannten Parametern. 


\section{Resultate der Oxidation von W-Spitzen mit $\mathrm{H}_{2} \mathrm{O}$}

In diesem Kapitel werden die Ergebnisse der Untersuchungen des Einflusses eines elektrischen Feldes auf das Oxidationsverhalten nanoskaliger Spitzen aus Wolfram vorgestellt. Dazu wird in einem einleitenden Abschnitt zunächst ein Überblick über die mit der Oxidbildung einhergehende Morphologieänderung der Spitzen gegeben. Es folgt die Darstellung der experimentellen Resultate der Kinetikmessungen sowie der Messungen zur Bestimmung von Struktur und Stöchiometrie der Oxidschichten. Abschließend werden die Ergebnisse der FEM-Rechnungen zur Bestimmung der elektrischen Feldstärke in der Umgebung der nanoskaligen Spitzen vorgestellt.

\subsection{Einführung des Oxidationseffekts}

Als Beispiel für den Einfluss eines elektrischen Feldes auf das Oxidationsverhalten nanoskaliger W-Spitzen zeigt Abb. 4.1 a) eine Folge von TEM-Aufnahmen einer Spitze, an die bei einem Druck von 10 mbar $\mathrm{H}_{2} \mathrm{O}$ für jeweils $10 \mathrm{~s}$ die angegebenen Spannungen angelegt wurden. Im Ausgangszustand $(0 \mathrm{~V})$ ist die natürliche, der Reaktion mit 2 Monolagen atomarem Sauerstoff entsprechende Oxiddicke [King1971] nicht erkennbar. Nach Anlegen einer Spannung von $250 \mathrm{~V}$ weist die Spitze im Apexbereich eine Oxidschicht auf. Die Dicke der Oxidschicht nimmt von $11 \mathrm{~nm}$ auf der Symmetrieachse mit zunehmendem Abstand vom Apex ab bis sie schließlich nicht mehr auflösbar ist. Die Grenzfläche zwischen Metall und Oxid ist dabei der ursprünglichen Spitzenform recht ähnlich. Nach

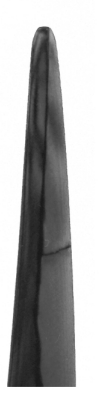

a) $\overline{100 \mathrm{~nm}} \quad 0$

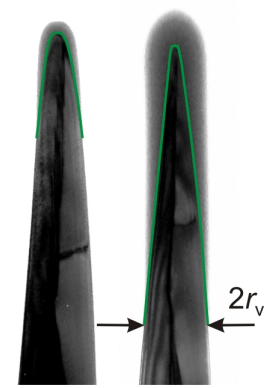

250

$400 \mathrm{~V}$

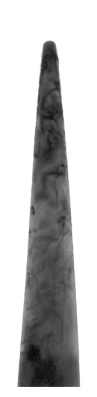

b) $\overline{100 \mathrm{~nm}} \quad 0$

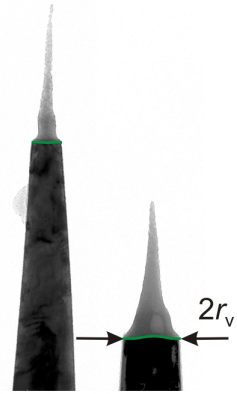

$450750 \mathrm{~V}$

Abbildung 4.1: Folge von TEM-Aufnahmen einer nanoskaligen W-Spitze, an die bei einem Druck von a) 10 mbar $\mathrm{H}_{2} \mathrm{O}$ und b) $10^{-3}$ mbar $\mathrm{H}_{2} \mathrm{O}$ für jeweils $10 \mathrm{~s}$ die angegebenen Spannungen angelegt wurden. Die Grenzfläche zwischen Metall und Oxid ist durch die grüne Linie hervorgehoben. Die deutlich erkennbaren Korngrenzen im Wolfram erlauben eine einheitliche Positionierung der Aufnahmen entlang der Symmetrieachse. 


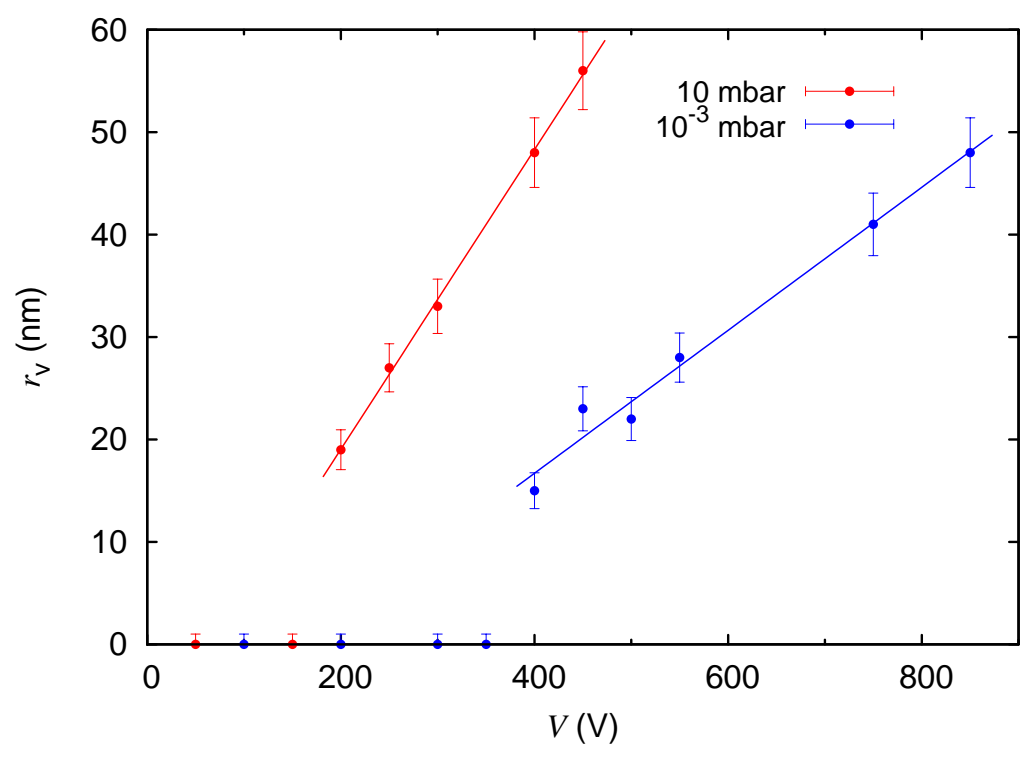

Abbildung 4.2: Auftragung des aus TEM-Aufnahmen bestimmten Wertes $r_{\mathrm{v}}$ gegen die an der Spitze anliegende Spannung $V$ für zwei Messreihen an W-Spitzen bei verschiedenem $\mathrm{H}_{2} \mathrm{O}-$ Druck. Die Spannung $V$ wurde in Schritten von $50 \mathrm{~V}$ erhöht und lag jeweils für $10 \mathrm{~s}$ an der Spitze an. Nach jedem Schritt wurde die Spitze mittels TEM charakterisiert.

Anlegen einer Spannung von 400 V zeigt sich ein qualitativ gleiches Bild, die Oxidschicht ist jedoch deutlich ausgedehnter. Die Schichtdicke auf der Symmetrieachse beträgt nun $59 \mathrm{~nm}$. Auch der Radius der Spitze am Ort verschwindender Oxiddicke $r_{\mathrm{v}}$ ist deutlich vergrößert. ${ }^{1}$

Erfolgt die Oxidationsreaktion bei einem geringeren Druck von $10^{-3}$ mbar $\mathrm{H}_{2} \mathrm{O}$, so weisen die Spitzen wie in Abb. 4.1 b) dargestellt eine gänzlich andere Morphologie auf. Nach Anlegen einer Spannung von $450 \mathrm{~V}$ wird die Ausbildung einer annähernd kegelförmigen Oxidspitze von $195 \mathrm{~nm}$ Höhe auf der W-Spitze beobachtet. Die Grenzfläche zwischen Metall und Oxid ist dabei nahezu ungekrümmt. Nachdem eine Spannung von $750 \mathrm{~V}$ angelegt wurde, zeigt sich qualitativ das gleiche Bild: Die Höhe der Oxidspitze ist unverändert, die wiederum nahezu ungekrümmte Grenzfläche zwischen Metall und Oxid und damit der Wert von $r_{\mathrm{v}}$ jedoch deutlich vergrößert. Der Vergleich mit dem Zustand vor Anlegen einer Spannung $(0 \mathrm{~V})$ zeigt darüberhinaus, dass die Oxidationsreaktion offenbar mit einem erheblichen Verlust von Spitzenmaterial einhergeht.

Die in den vorausgehenden Absätzen beschriebenen Resultate machen deutlich, dass unter dem Einfluss eines externen elektrischen Feldes die Oxidation von nanoskaligen W-Spitzen weitaus stärker als im feldfreien erfolgt. Zur Darstellung der Abhängigkeit der Oxidationsreaktion vom elektrischen Feld ist in Abb. 4.2 der Wert $r_{\mathrm{v}}$ als Maß für den Grad der Oxidbildung gegen die Spannung $V$ aufgetragen. Für einen Druck von 10 mbar $\mathrm{H}_{2} \mathrm{O}$ wird bei Spannungen unterhalb von $200 \mathrm{~V}$ keine Oxidschicht auf den Spitzen gefunden $\left(r_{\mathrm{v}}=0\right)$. Nach Anlegen einer Spannung von $200 \mathrm{~V}$ ist dann deutlich eine Oxidschicht mit $r_{\mathrm{v}}=19 \mathrm{~nm}$ auf der W-Spitze erkennbar, und mit schrittweise zunehmender Span-

\footnotetext{
${ }^{1}$ Die Position $r_{\mathrm{v}}$ bezeichnet jeweils den Radius, an dem die Dicke des unter dem Einfluss des elektrischen Feldes gebildeten Oxides verschwindet. Das natürliche Oxid auf der Spitzenoberfläche bleibt dabei unberücksichtigt.
} 


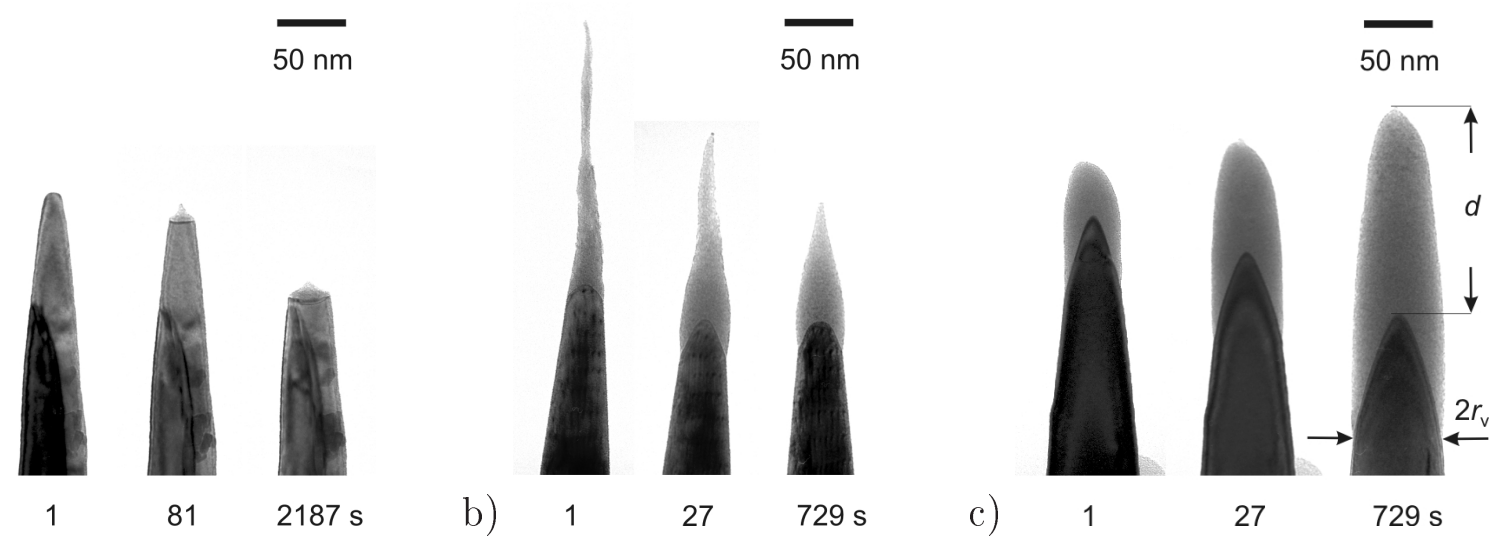

Abbildung 4.3: Folgen von TEM-Aufnahmen dreier W-Spitzen, nachdem eine Spannung von $350 \mathrm{~V}$ für die angegebenen Zeiten bei einem Druck von a) $10^{-4}$ mbar, b) $10^{-2}$ mbar und c) 1 mbar $\mathrm{H}_{2} \mathrm{O}$ angelegt wurde.

nung steigt auch der Wert von $r_{\mathrm{v}}$ monoton an. Bei einem Druck von $10^{-3}$ mbar $\mathrm{H}_{2} \mathrm{O}$ wird ein ähnliches Verhalten beobachtet. Die Ausbildung einer Oxidschicht erfolgt jedoch erst ab Spannungen von mindestens $400 \mathrm{~V}$ und der Anstieg von $r_{\mathrm{v}}$ mit zunehmender Spannung ist nicht so stark wie bei einem Druck von 10 mbar $\mathrm{H}_{2} \mathrm{O}$.

Somit existiert für Wolfram offenbar eine kritische Feldstärke, oberhalb derer sich Oxidschichten ausbilden. Die Dicke der unter dem Einfluss eines externen Feldes entstehenden Oxidschichten ist dabei erheblich größer als die natürliche Oxiddicke. Da die Ausgangsmorphologie der nanoskaligen Spitzen wegen der in 3.1.1 geschilderten Präparationsmethode vergleichbar ist, folgt aus Abb.4.2, dass die kritische Feldstärke vom $\mathrm{H}_{2} \mathrm{O}$-Druck abhängt. Bei geringerem Druck nimmt sie offenbar größere Werte an. Quantitative Angaben zum Betrag der kritischen Feldstärke in Abhängigkeit vom $\mathrm{H}_{2} \mathrm{O}$-Druck werden in 4.4 gemacht. Im folgenden Abschnitt wird zunächst die Kinetik der Oxidationsreaktion betrachtet.

\subsection{Kinetik der Oxidationsreaktion}

In diesem Abschnitt wird zunächst die zeitliche Entwicklung der Morphologie der nanoskaligen Spitzen beschrieben. Dabei ist insbesondere von Interesse, ob ebenso wie bei der natürlichen Oxidation von Wolfram das Oxidwachstum bei Erreichen einer gewissen Schichtdicke im Rahmen der Messgenauigkeit zu Erliegen kommt. Da nach 2.2.1 die Oxidationsreaktion stets mit einem Nettotransport positiver Ladung von der Metall-OxidGrenzfläche zu der Oxidoberfläche einhergeht, kann die Kinetik der Oxidationsreaktion auch in situ durch eine Strommessung verfolgt werden. Entsprechende Messergebnisse werden im zweiten Teil dieses Abschnittes vorgestellt.

\subsubsection{Kinetik der Morphologieentwicklung}

Eine Übersicht über die Kinetik der Morphologieentwicklung für unterschiedlichen $\mathrm{H}_{2} \mathrm{O}$ Druck zeigt Abb. 4.3. Bei einem Druck von 1 mbar wird bereits nach $1 \mathrm{~s}$ die Ausbildung 


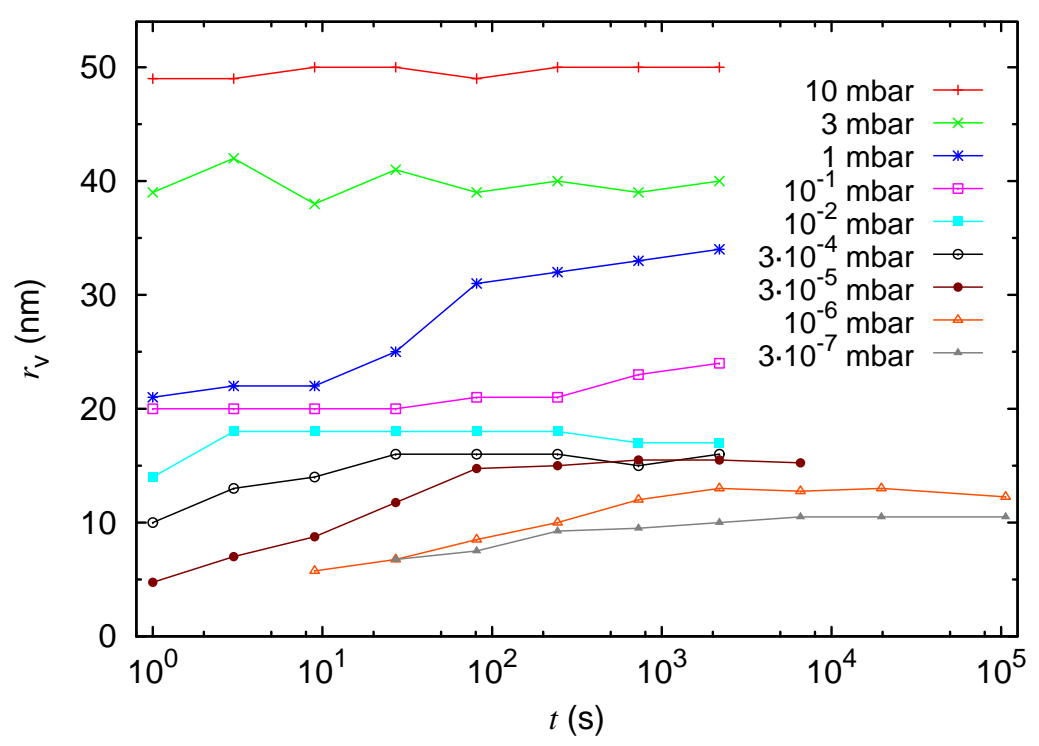

Abbildung 4.4: Zeitlicher Verlauf des aus TEM-Aufnahmen bestimmten Wertes $r_{\mathrm{v}}$ für Messreihen an W-Spitzen bei verschiedenem $\mathrm{H}_{2} \mathrm{O}$-Druck. An den Spitzen lag je eine Spannung von $350 \mathrm{~V}$ an. ${ }^{2}$ Die Genauigkeit der Werte liegt bei etwa $10 \%$, aus Gründen der Übersichtlichkeit sind jedoch keine Fehler angegeben.

einer Oxidschicht im Apexbereich der Spitze beobachtet. Für längere Zeiten nehmen sowohl $r_{\mathrm{v}}$ als auch die Dicke der Oxidschicht auf der Symmetrieachse $d$ monoton zu. Liegt der $\mathrm{H}_{2} \mathrm{O}$-Druck bei $10^{-2}$ mbar, so bildet sich innerhalb von $1 \mathrm{~s}$ ebenfalls eine erhebliche Menge Oxid, im Gegensatz zur Morphologie bei 1 mbar allerdings in Form eines spitzen Kegels mit $d=194 \mathrm{~nm}$ und einem Krümmungsradius von etwa $2 \mathrm{~nm}$. Für längere Zeiten nimmt $r_{\mathrm{v}}$ wiederum zu, $d$ hingegen verringert sich. Der Krümmungsradius an der Spitze des Oxidkegels ändert sich kaum. Bei einem noch geringeren Druck von $10^{-4}$ mbar $\mathrm{H}_{2} \mathrm{O}$ wird während der ersten $20 \mathrm{~s}$ keine Änderung der Spitzenmorphologie beobachtet. Nach hinreichend langer Zeit bildet sich im Apexbereich eine Oxidschicht in Form eines stumpfen Kegels, der Krümmungsradius an der Spitze des Oxides liegt wiederum bei etwa $2 \mathrm{~nm}$. Auch hier nimmt $r_{\mathrm{v}}$ mit der Zeit zu, $d$ hingegen ändert sich kaum.

Zur quantitativen Beschreibung der Kinetik der Oxidationsreaktion ist in Abb. 4.4 der aus den TEM-Aufnahmen bestimmte Wert von $r_{\mathrm{v}}$ gegen die Zeit aufgetragen. Dabei zeigt sich, dass für eine jeweils feste Zeit der Wert von $r_{\mathrm{v}}$ monoton mit dem $\mathrm{H}_{2} \mathrm{O}-$ Druck zunimmt. Auch für jeweils festen Druck wird eine monotone Zunahme von $r_{\mathrm{v}}$ mit der Zeit beobachtet. Liegt der $\mathrm{H}_{2} \mathrm{O}$-Druck bei mindestens 3 mbar, so kommt die Oxidationsreaktion offenbar bereits nach weniger als $1 \mathrm{~s}$ zum Erliegen und es wird ein im

\footnotetext{
${ }^{2}$ In sämtlichen Messungen bei einem $\mathrm{H}_{2} \mathrm{O}$-Druck von weniger als $10^{-4}$ mbar wurde eine Spannung von $700 \mathrm{~V}$ an die Spitzen angelegt, um noch Oxidschichten hinreichender Größe zu erhalten. Zur Gewährleistung der Vergleichbarkeit mit den übrigen Daten wurden die abgebildeten TEM-Aufnahmen dieser Spitzen sowie die daraus bestimmten Werte $r_{\mathrm{v}}$ und $d$ je mit einem Faktor $\frac{1}{2}$ skaliert. Die Berechnungen mit der Finite-Elemente-Methode erfolgten hingegen unter Berücksichtigung der tatsächlichen Größen- und Spannungsverhältnisse. Für die Darstellung der Feldverteilung in Abb. 4.14 und Abb.4.16 für Druckwerte kleiner als $10^{-4}$ mbar wurde jedoch wiederum eine Skalierung mit einem Faktor $\frac{1}{2}$ vorgenommen.
} 


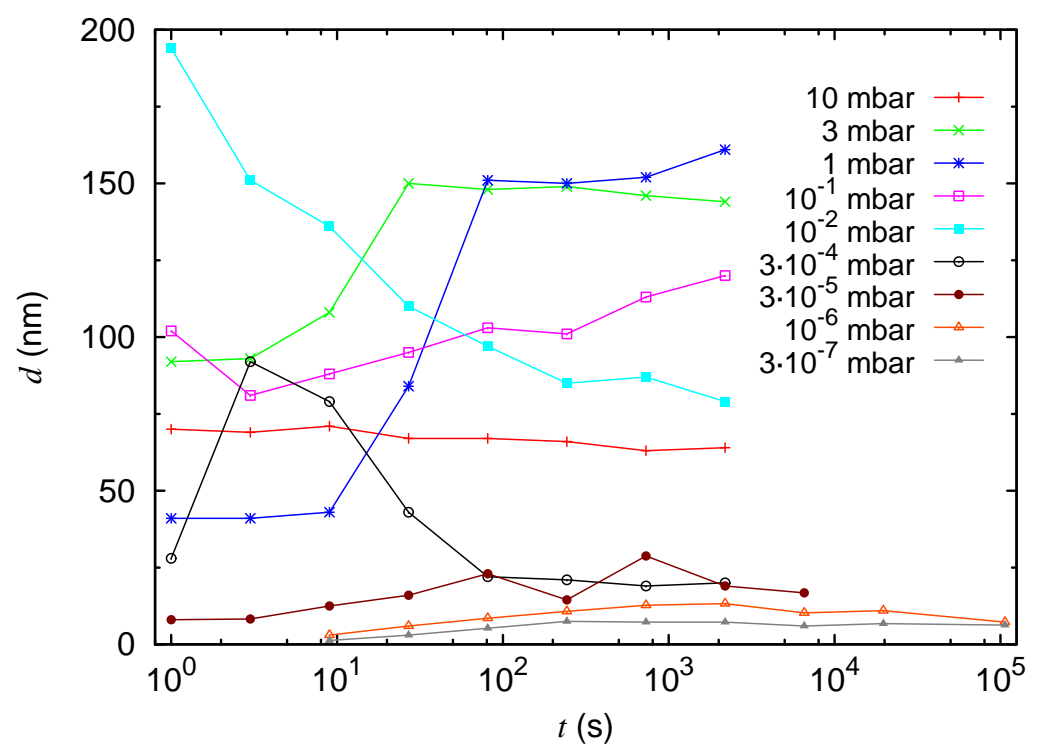

Abbildung 4.5: Zeitlicher Verlauf des aus TEM-Aufnahmen bestimmten Wertes $d$ für Messreihen bei verschiedenem $\mathrm{H}_{2} \mathrm{O}$-Druck. Für weitere Angaben siehe Abb. 4.4.

Rahmen der Messgenauigkeit konstanter Wert für $r_{\mathrm{v}}$ gefunden. Für Druckwerte zwischen $10^{-1}$ mbar und 1 mbar $\mathrm{H}_{2} \mathrm{O}$ wird hingegen auch nach $10^{3} \mathrm{~s}$ noch eine Zunahme von $r_{\mathrm{v}}$ beobachtet. Liegt der $\mathrm{H}_{2} \mathrm{O}$-Druck noch tiefer, so kommt nach einer Phase der Zunahme von $r_{\mathrm{v}}$ das Oxidwachstum für große Zeiten wieder zum Erliegen.

Die zeitliche Entwicklung der Dicke der Oxidschicht auf der Symmetrieachse $d$ für dieselben Proben zeigt Abb. 4.5. Das Verhalten für kurze Zeiten wird offenbar durch mindestens zwei konkurrierende Prozesse bestimmt. Für einen $\mathrm{H}_{2} \mathrm{O}$-Druck von über $10^{-1}$ mbar wird im Wesentlichen eine Zunahme von $d$ mit der Zeit beobachtet. Bei einem Druck zwischen $10^{-4}$ mbar und $10^{-1}$ mbar konkurriert hingegen ein schneller Prozess, welcher innerhalb von Sekunden zur Ausbildung von bis zu $200 \mathrm{~nm}$ langen Oxidspitzen führt, mit einem langsameren Prozess, welcher eine Verringerung von $d$ zur Folge hat. Für Druckwerte von weniger als $10^{-4}$ mbar $\mathrm{H}_{2} \mathrm{O}$ dominiert dann wiederum die stetige Zunahme von $d$. Beträgt der $\mathrm{H}_{2} \mathrm{O}$-Druck jedoch mindestens 3 mbar oder höchstens $3 \cdot 10^{-4}$ mbar, so deutet auch der zeitliche Verlauf von $d$ darauf hin, dass die Oxidationsreaktion nach spätestens $10^{3}$ s zum Erliegen kommt.

Die sich für große Zeiten $\left(t>10^{3} \mathrm{~s}\right)$ in Abhängigkeit vom $\mathrm{H}_{2} \mathrm{O}$-Druck einstellende Spitzenmorphologie ist in Abb. 4.6 dargestellt. Dabei ist nach den Ausführungen des vorigen Absatzes die Morphologie für die meisten Druckwerte zeitlich stabil, lediglich im Bereich von $10^{-2}$ mbar bis 1 mbar $\mathrm{H}_{2} \mathrm{O}$ verläuft die Oxidationsreaktion noch mit signifikanter Rate. Weiterhin geht aus Abb. 4.6 hervor, dass sich die Krümmung der Metall-Oxid-Grenzfläche mit abnehmendem $\mathrm{H}_{2} \mathrm{O}$-Druck von konvex zu konkav in Bezug auf die metallische Spitze ändert. Eine in Zylinderkoordinaten definierte Funktion, welche die Oxidoberfläche beschreibt, wechselt mit abnehmendem Druck ihre Krümmung von konkav zu konvex. 


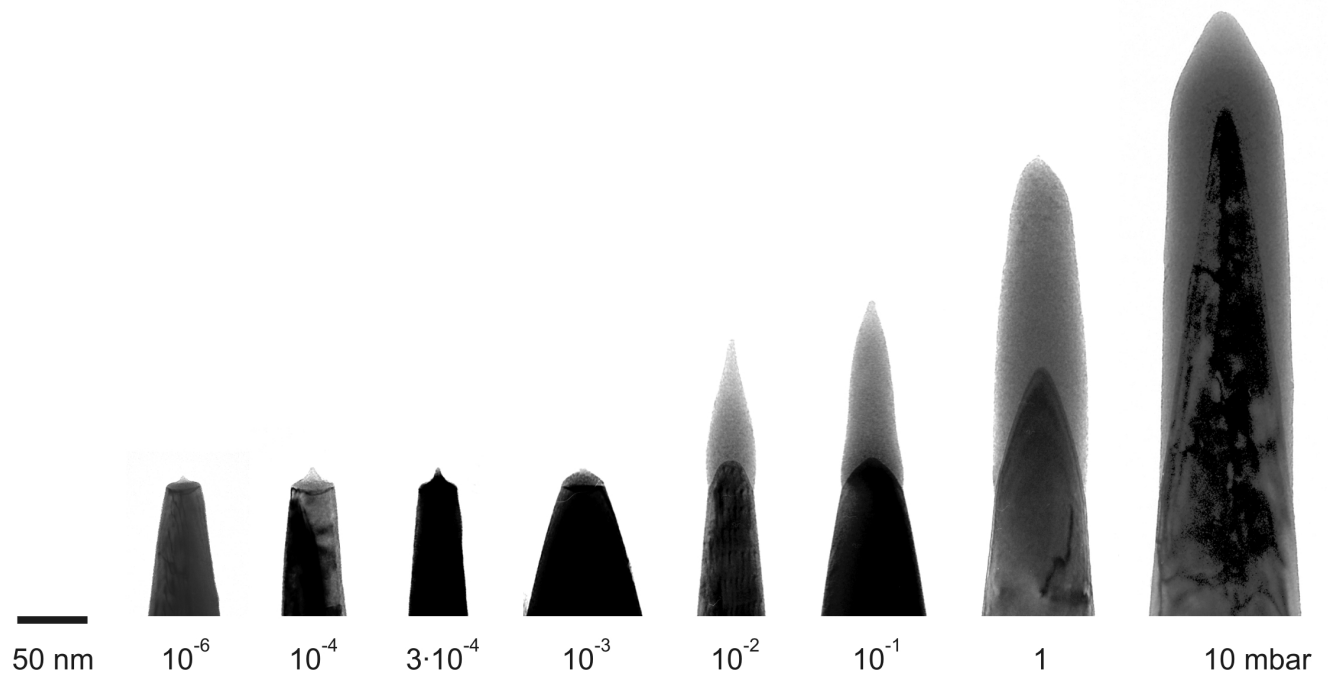

Abbildung 4.6: TEM-Aufnahmen verschiedener W-Spitzen, nachdem bei dem angegebenen $\mathrm{H}_{2} \mathrm{O}-$ Druck eine Spannung von $350 \mathrm{~V}$ für mindestens $10^{3} \mathrm{~s}$ angelegt wurde. Bei einem Druck im Bereich von $10^{-2}$ mbar bis 1 mbar erfolgen für größere Zeiten noch signifikante Änderungen der Morphologie, für höhere und tiefere Drücke bleibt die Morphologie unverändert.

\subsubsection{Kinetik des Reaktionsstromes}

Während der Oxidationsreaktion lässt sich ein Strom positiver Ladungen messen, welcher von der Gegenelektrode zur Erde fließt. Der zeitliche Verlauf dieses Stromes $I$ ist in Abb. 4.7 dargestellt. Unabhängig vom $\mathrm{H}_{2} \mathrm{O}$-Druck treten zwei Regime auf: Nach dem

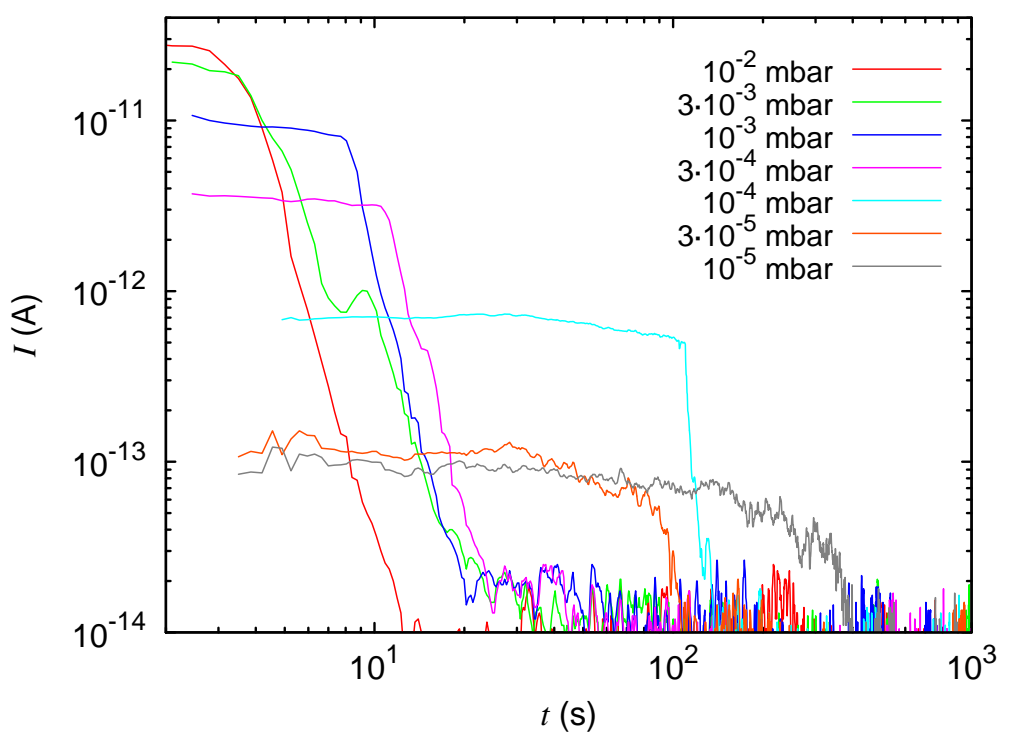

Abbildung 4.7: Zeitlicher Verlauf des Stromes, welcher während der Oxidationsreaktion zwischen Gegenelektrode und Erde gemessen wird (siehe Abb. 3.2). In der doppelt logarithmischen Darstellung ist der um kapazitive Effekte beim Anlegen der Spannung $V=700 \mathrm{~V}$ bereinigte Netto-Strom dargestellt, daher sind für kurze Zeiten keine validen Werte verfügbar. Der zugängliche Bereich des $\mathrm{H}_{2} \mathrm{O}$-Druckes von $10^{-5}$ mbar bis $10^{-2}$ mbar ist durch die Zeit- und Stromaufösung des Amperemeters begrenzt. 


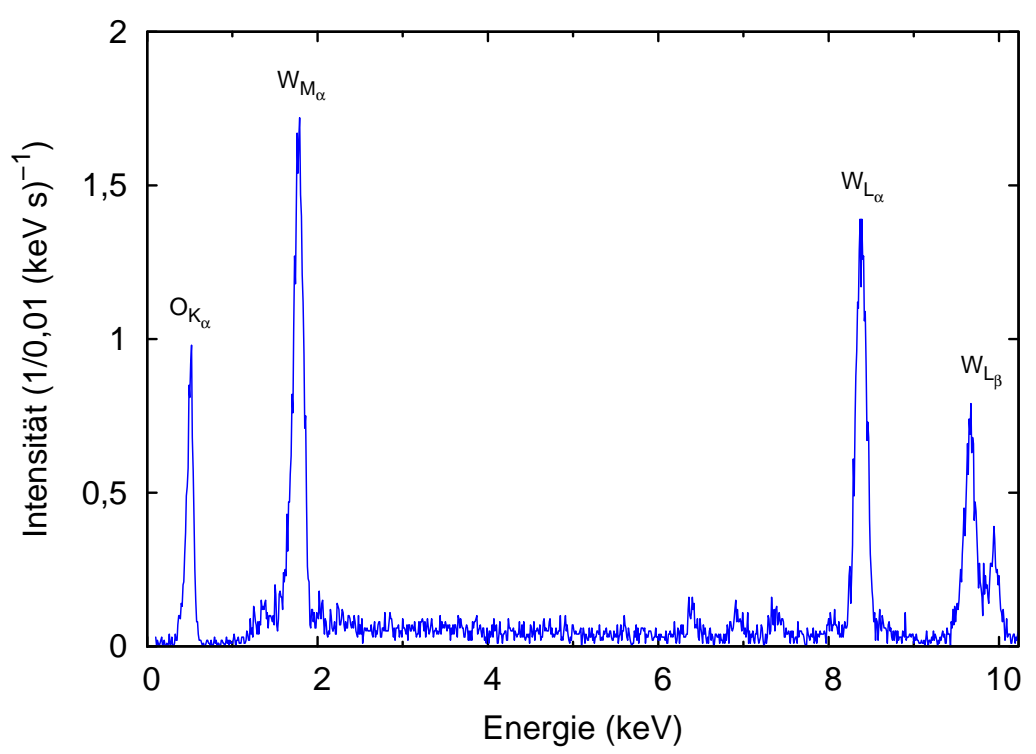

Abbildung 4.8: EDX-Spektrum einer Oxidschicht mit charakteristischen Linien für Sauerstoff und Wolfram. Die schwachen Linien zwischen $6 \mathrm{keV}$ und $8 \mathrm{keV}$ resultieren aus der Wechselwirkung der primären Röntgenstrahlung mit dem TEM-Probenhalter.

Anlegen der Spannung $V$ fließt zunächst ein nahezu konstanter Strom $I_{\mathrm{p}}$. Nach einer vom Druck abhängigen Zeit $t_{\mathrm{s}}$ fällt der Strom dann auf einen Wert unterhalb des Rauschpegels der Messung ab. Da die Oxidationsreaktion nach 2.2.1 stets mit einem Transport positiver Ladung von der Metall-Oxid-Grenzfläche zu der Oxidoberfläche verbunden ist, deutet somit auch die Strommessung darauf hin, dass die Oxidationsreaktion nach einer gewissen Zeit zum Erliegen kommt.

\subsection{Struktur und Stöchiometrie des Oxides}

In diesem Abschnitt werden Messungen zur chemischen Charakterisierung der sich unter dem Einfluss eines elektrischen Feldes auf den Spitzen ausbildenden Schichten vorgestellt. Sie belegen, dass tatsächlich eine Oxidbildung erfolgt.

\subsubsection{Stöchiometrie des Oxides}

Zur Bestimmung der chemischen Zusammensetzung der Schichten wurden zunächst EDX-Analysen vorgenommen, ${ }^{3}$ ein typisches Spektrum zeigt Abb. 4.8. Neben den Lund M-Linien für Wolfram tritt mit hoher Intensität die K-Linie für Sauerstoff auf und deutet auf das Vorliegen eines Oxides hin. Da bedingt durch die Spitzenmorphologie Absorptions- und Fluoreszenzeffekte schwer zu quantifizieren sind, ist eine Bestimmung der Oxidstöchiometrie aus den gemessenen Intensitätsverhältnissen jedoch nicht möglich.

\footnotetext{
${ }^{3}$ Die EDX-Analysen wurden an Spitzen durchgeführt, bei denen die Oxidationsreaktion nicht unter reiner $\mathrm{H}_{2} \mathrm{O}$-Atmosphäre, sondern unter Umgebungsatmosphäre bei Umgebungsdruck erfolgte. Bei einer typischen relativen Luftfeuchtigkeit entspricht dies einem Partialdruck von $\mathrm{H}_{2} \mathrm{O}$ in der Größenordnung von 10 mbar.
} 


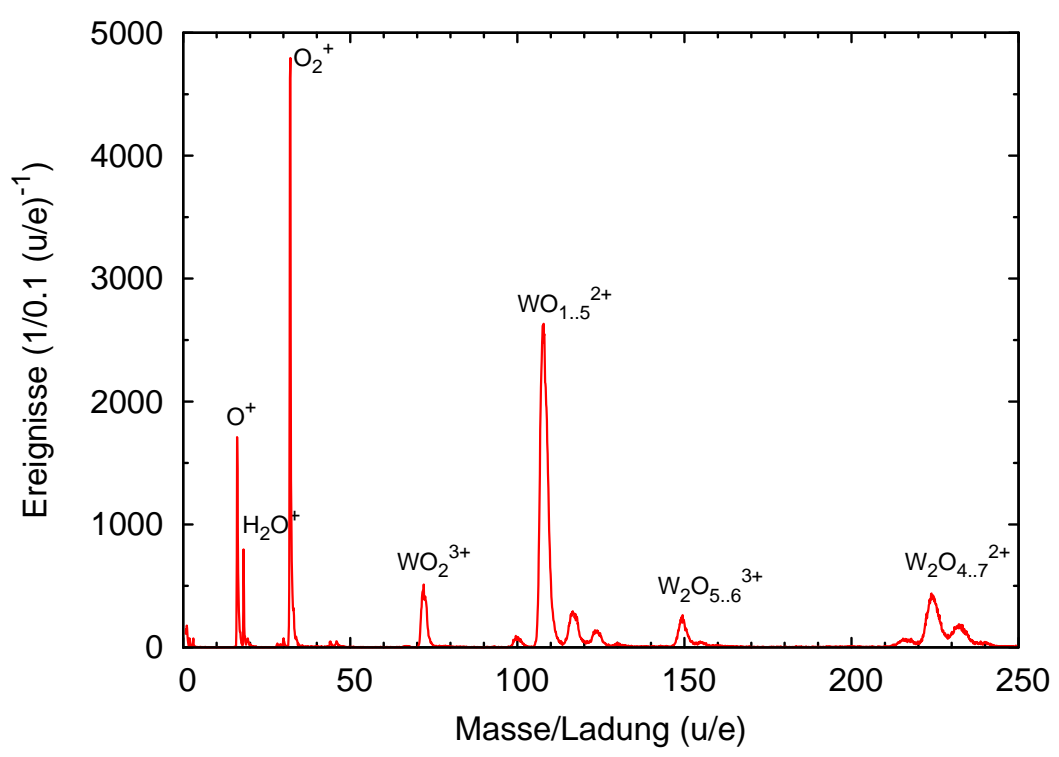

Abbildung 4.9: Mit Laser-unterstützter Atomsondentomographie ermitteltes Massenspektrum einer bei einem Druck von $10^{-1}$ mbar $\mathrm{H}_{2} \mathrm{O}$ hergestellten Oxidschicht. Die Oxidionen treten stets in Gruppen mit einer unterschiedlichen Zahl von Sauerstoffatomen auf. Für jede Gruppe sind jeweils die Ionen mit der geringsten Ladungszahl angegeben, welche dem gemessenen Masse-Ladungs-Verhältnis entsprechen.

Ebenso kann gegebenenfalls vorhandener Wasserstoff mittels EDX nicht nachgewiesen werden. Zur quantitativen chemischen Charakterisierung der Oxidschichten wurde daher Laser-unterstützte Atomsondentomographie eingesetzt. Ein Massenspektrum einer Oxidschicht, welche durch Oxidation der Spitze bei einem $\mathrm{H}_{2} \mathrm{O}$-Druck von $10^{-1}$ mbar erzeugt wurde, zeigt Abb.4.9. Das Auftreten einer Vielzahl von Peaks macht deutlich, dass unter den gewählten Analysebedingungen offenbar keine Feldverdampfung einzelner Atome erfolgt, sondern mehratomige Moleküle von der Spitze abgelöst werden. Alle in dem Massenspektrum auftretenden Peaks lassen sich $\mathrm{W}_{\mathrm{x}} \mathrm{O}_{\mathrm{y}}$ sowie $\mathrm{H}_{2} \mathrm{O}$ und $\mathrm{H}_{3} \mathrm{O}$ zuordnen. Wegen der zahlreichen Isotope des Wolframs reicht bei den Oxidmolekülen die Massenauflösung jedoch nicht aus, um Oxide von Hydroxiden zu trennen. $\mathrm{H}_{2} \mathrm{O}^{+}$und $\mathrm{H}_{3} \mathrm{O}^{+}$-Peaks in der beobachteten Größe können auch auf Ionisation von adsorbiertem Restgas während der Analyse zurückzuführen sein. Somit kann nicht festgestellt werden, ob in gewissem Umfang auch Wolframhydroxid durch die Oxidationsreaktion gebildet wird. Da bei feldfreier Oxidation von Wolfram an Laborluft Wolframhydroxid lediglich an der Oxidoberfläche, nicht jedoch im Volumenoxid gefunden wird [Barr1978], wird in den weiteren Betrachtungen ein Wasserstoffanteil im gebildeten Oxid vernachlässigt.

Eine Rekonstruktion der Oxidschicht, deren Massenspektrum in Abb. 4.9 dargestellt ist, zeigt Abb. 4.10. Im rechten Teil der Abbildung ist die dreidimensionale Anordnung der Wolfram- und Sauerstoffatome dargestellt. Die im Massenspektrum identifizierten Moleküle wurde dazu jeweils in ihre atomaren Bestandteile aufgespalten. Der linke Teil der Abbildung zeigt eine TEM-Aufnahme der nanoskaligen W-Spitze vor der Analyse mittels Laser-unterstützter Atomsondentomographie mit der maßstabsgetreuen Lage des Rekonstruktionsvolumens von $20 \times 20 \times 35 \mathrm{~nm}^{3}$. In der Rekonstruktion erscheint die Oxidschicht kompakt und homogen. Um quantitative Informationen der Zusammensetzung zu erhalten, wurde ein Konzentrationsprofil entlang der Symmetrieachse der Spitze ermit- 


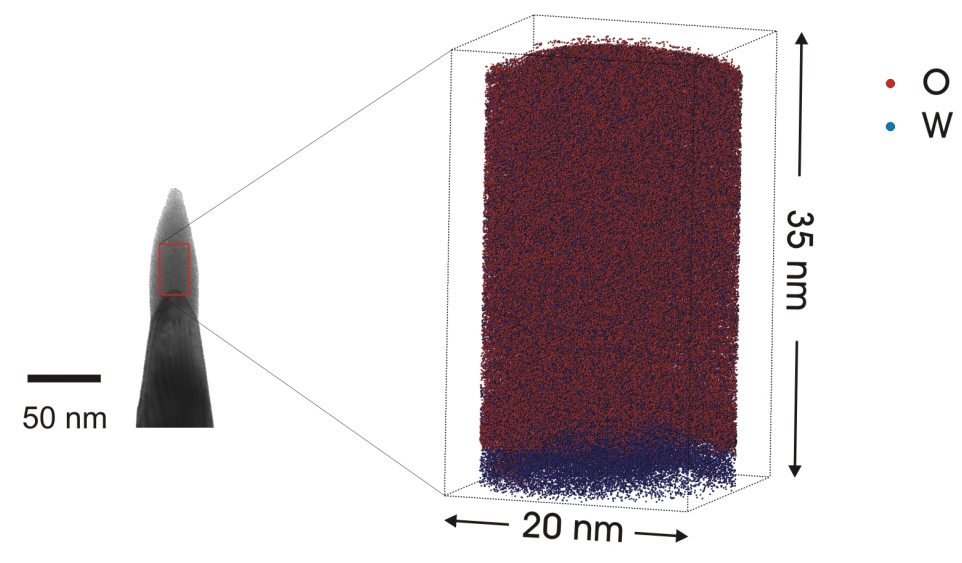

\begin{abstract}
Abbildung 4.10: Rekonstruktion einer Oxidschicht, welche durch Oxidation einer Spitze bei $10^{-1} \mathrm{mbar}$ $\mathrm{H}_{2} \mathrm{O}$ erzeugt wurde. Die jeweiligen Atompositionen sind durch farbige Punkte markiert. Der linke Teil der Abbildung zeigt die Position des zylindrischen Rekonstruktionsvolumens in einer TEM-Aufnahme der Spitze. In dem Massenspektrum in Abb. 4.9 sind lediglich die Moleküle der Oxidschicht dargestellt, die Atome der metallischen W-Spitze gehen nicht ein.
\end{abstract}

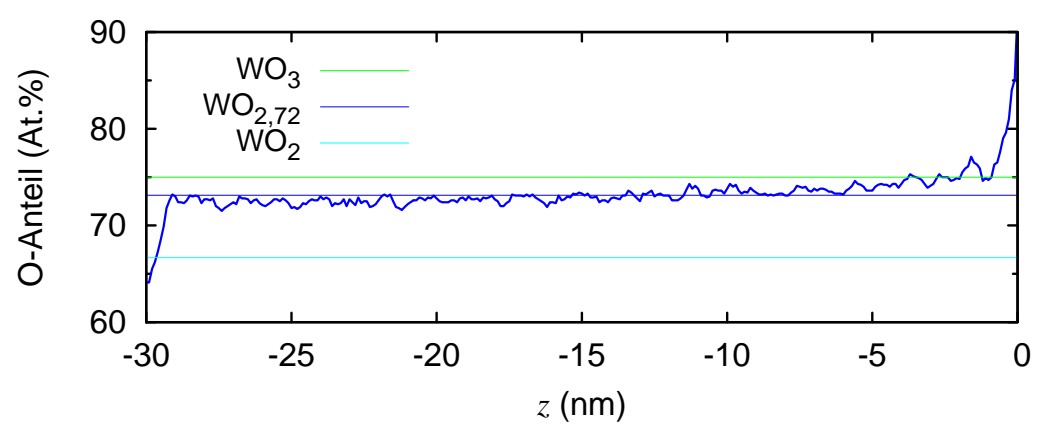

Abbildung 4.11: Konzentrationsprofil der in Abb. 4.10 dargestellten Oxidschicht. Bestimmt wurde die Sauerstoffkonzentration entlang eines Zylinders mit einem Radius von $7 \mathrm{~nm}$, dessen Achse mit der Symmetrieachse der Spitze zusammenfällt. Nahe der Oxidoberfläche wird in Übereinstimmung mit [Barr1978] ein Sauerstoffanteil von 75 At.\% entsprechend dem Oxid mit dem höchsten Oxidationszustand $\mathrm{WO}_{3}$ gefunden. Im Volumen des Oxides beträgt die Sauerstoffkonzentration 73(2) At.\% und entspricht damit dem thermodynamisch erst oberhalb von $858 \mathrm{~K}$ stabilen $\mathrm{WO}_{2,72}$ [Pred1998].

telt, dargestellt in Abb. 4.11. Im Oxid liegt der Sauerstoffanteil demnach nahezu konstant bei 73(2) At.\%, der steile Anstieg bei $z=-1 \mathrm{~nm}$ markiert die Oxidoberfläche, der Abfall bei $z=-29 \mathrm{~nm}$ den Übergang zum metallischen Wolfram. Die Stöchiometrie des Oxides liegt somit nahe bei $\mathrm{WO}_{3}$, wobei eine geringfügige Unsicherheit in der Stöchiometriebestimmung aus zwei Gründen resultiert: Zum einen können durch die Flugzeitmessung nur Ionen mir einem Verhältnis von Masse zu Ladung von höchstens $250 \mathrm{u} / \mathrm{e}$ gemessen werden. Sollten darüber hinaus noch in signifikantem Maße Ionen auftreten, kann die tatsächliche Stöchiometrie von der gemessenen abweichen. Weiterhin ist, wie in Abb. 4.9 angegeben, die Zuordnung der Ionen zu den entsprechenden Peaks des Massenspektrums nicht eindeutig. So kann etwa der Peak bei $232 \mathrm{u}$ /e dem Auftreten von $\mathrm{W}_{2} \mathrm{O}_{6}^{2+}$ oder aber $\mathrm{WO}_{3}^{+}$zugeschrieben werden, wodurch sich jedoch die Zahl der Atome und damit auch der Einfluss des Peaks auf die Stöchiometrie ändert. Aus einer Betrachtung der mit der Oxidationsreaktion einhergehenden Volumenänderungen von Metall und Oxid in 6.1.3 


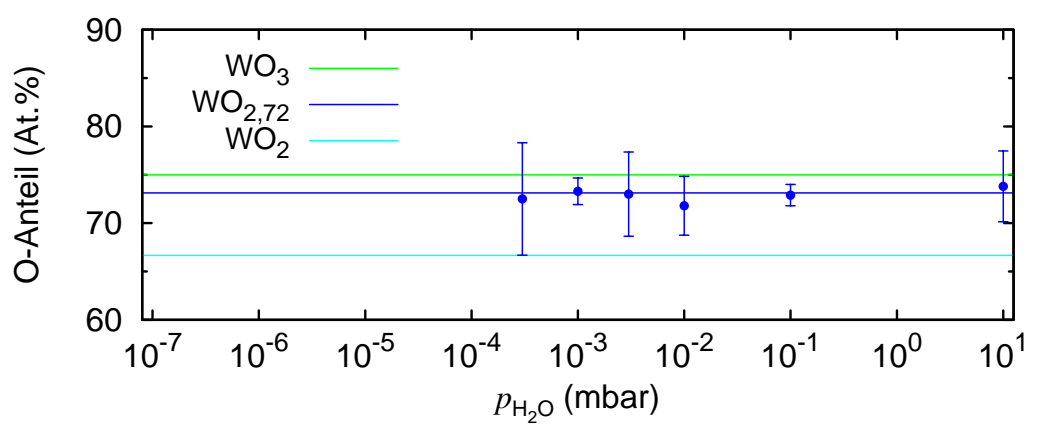

Abbildung 4.12: Mit Laser-unterstützter Atomsondentomographie ermittelter Sauerstoffanteil in der Oxidschicht in Abhängigkeit vom $\mathrm{H}_{2} \mathrm{O}$-Druck während der Oxidationsreaktion.

folgt jedoch, dass die tatsächliche Stöchiometrie nahe der mittels Laser-unterstützter Atomsondentomographie ermittelten liegt. Für die in Abb. 4.10 dargestellte Oxidschicht folgt als Mittelwert für die gesamte Oxidschicht eine Stöchiometrie von $\mathrm{WO}_{2,7(2)}$.

Um den Einfluss der Wachstumsbedingungen auf die Stöchiometrie des gebildeten Oxides aufzuklären, wurden bei unterschiedlichem $\mathrm{H}_{2} \mathrm{O}$-Druck gebildete Oxidschichten mit Laser-unterstützter Atomsondentomographie untersucht. Eine Auftragung des Sauerstoffanteils der Oxidschichten in Abhängigkeit vom $\mathrm{H}_{2} \mathrm{O}$-Druck $p_{\mathrm{H}_{2} \mathrm{O}}$ in Abb. 4.12 zeigt, dass unabhängig vom Druck eine Oxidstöchiometrie nahe bei $\mathrm{WO}_{2,72}$ gefunden wird. Die chemische Zusammensetzung der Oxidschichten hängt somit offenbar nicht von den Wachstumsbedingungen ab.

\subsubsection{Struktur des Oxides}

Die Bestimmung der Struktur der Oxidschichten erfolgt mittels Feinbereichs- und Feinstrahlbeugung. Dabei werden unabhängig von den experimentellen Parametern wie $\mathrm{H}_{2} \mathrm{O}-$ Druck, Spannung und Reaktionsdauer qualitativ gleiche Beugungsbilder gefunden. Ein Feinbereichs-Beugungsbild einer W-Spitze, an der die Oxidationsreaktion bei 1 mbar $\mathrm{H}_{2} \mathrm{O}$ erfolgte, zeigt Abb. $4.13 \mathrm{a}$ ). Da trotz des geringen Oxidvolumens nahezu kontinuierliche Beugungsringe auftreten, liegt das Oxid offenbar in Form nanometergroßer Kris-

a)

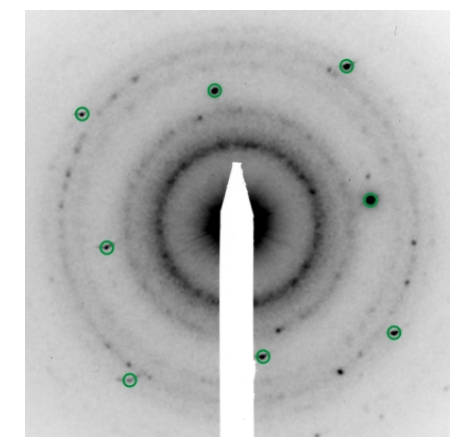

b) $\overline{50 \mathrm{~nm}}$

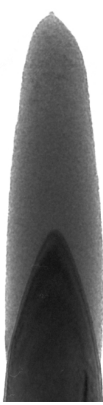

Abbildung 4.13: a) Feinbereichs-Beugungsbild der in der TEM-Aufnahme in b) abgebildeten Spitze. An die W-Spitze wurde zuvor bei einem Druck von 1 mbar für 2187 s eine Spannung von $350 \mathrm{~V}$ angelegt. Mit grünen Kreisen markiert ist das Beugungsbild der einkristallinen W-Spitze, die in geringster Entfernung zum Nullstrahl liegenden Reflexe sind vom Typ $\{200\}$. 

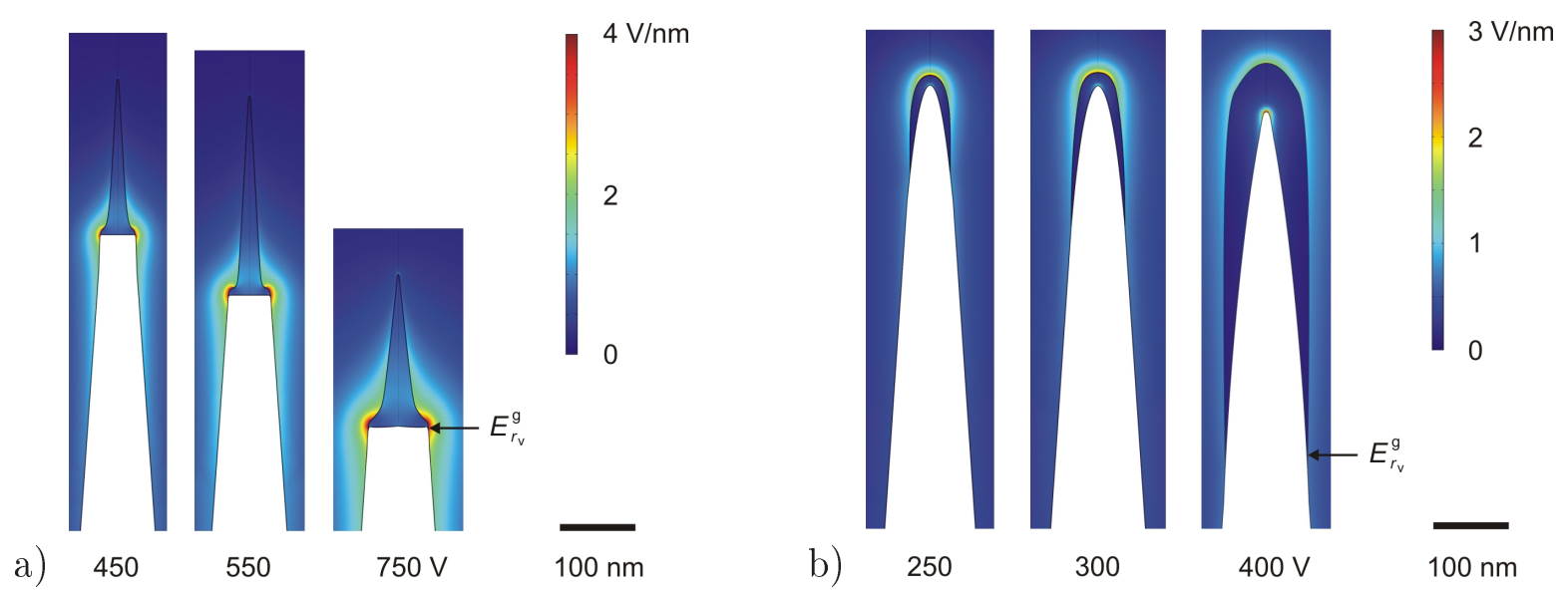

\begin{abstract}
Abbildung 4.14: Mit der Finite-Elemente-Methode berechnete Feldstärkeverteilung in der Umgebung zweier Spitzen, nachdem bei einem $\mathrm{H}_{2} \mathrm{O}$-Druck von a) $10^{-3}$ mbar und b) 10 mbar für jeweils $10 \mathrm{~s}$ die angegebene Spannung angelegt wurde. Der Betrag der Feldstärke ist durch die jeweils angegebene Farbskala kodiert. $E_{r_{\mathrm{v}}}^{\mathrm{g}}$ bezeichnet die Feldstärke direkt an der Oxidoberfläche im Gasraum am Ort verschwindender Oxiddicke $r_{\mathrm{v}}$.
\end{abstract}

tallite vor; auch eine gewisse Körnigkeit des in Abb. 4.13 b) dargestellten Hellfeldbildes der Oxidschicht weist darauf hin.

\title{
4.4 Feldstärkeverteilung im Oxid
}

Zur Berechnung der Feldstärkeverteilung in der Umgebung der nanoskaligen Spitzen mit Hilfe der Finite-Elemente-Methode muss zunächst die Permittivitätszahl $\varepsilon_{\mathrm{r}}$ des gebildeten Oxides bekannt sein. Da an den nanoskaligen Spitzen keine experimentelle Bestimmung von $\varepsilon_{\mathrm{r}}$ vorgenommen werden konnte, wird für die Berechnungen ein Wert von $\varepsilon_{\mathrm{r}}=10$ zugrunde gelegt. Dies entspricht in guter Näherung dem aus der Literatur [Hann1983] bekannten Wert von $\varepsilon_{\mathrm{r}}$ für nahezu stöchiometrisches $\mathrm{WO}_{3}$.

\subsubsection{Feldstärkeverteilung in Abhängigkeit vom Potential}

Für die in 4.1 abgebildeten Spitzen zeigt Abb. 4.14 die Feldstärkeverteilung in der Umgebung der Spitzen bei Variation der an den Spitzen anliegenden Spannung. Es treten elektrische Felder mit einer Stärke von bis zu $4 \mathrm{~V} / \mathrm{nm}$ auf. Erfolgt die Oxidationsreaktion bei einem Druck von 10 mbar $\mathrm{H}_{2} \mathrm{O}$, so zeigt sich nach Abb. $4.14 \mathrm{~b}$ ) unabhängig von der an der Spitze anliegenden Spannung ein qualitativ gleiches Bild: Innerhalb der Oxidschicht liegt die Feldstärke nahezu überall deutlich unterhalb von $1 \mathrm{~V} / \mathrm{nm}$. Lediglich an der Grenzfläche zum Metall nahe der Symmetrieachse wird dieser Wert wegen der starken Krümmung deutlich überschritten. Direkt an der Oxidoberfläche im Gasraum hingegen werden wegen der sprunghaften Änderung der Permittivitätszahl überall Feldstärken im Bereich von $1 \mathrm{~V} / \mathrm{nm}$ bis $2 \mathrm{~V} / \mathrm{nm}$ erreicht. Dabei nimmt die Feldstärke mit zunehmendem Abstand vom Apex ab. Beträgt der $\mathrm{H}_{2} \mathrm{O}$-Druck während der Oxidationsreaktion lediglich $10^{-3}$ mbar, so zeigt sich nach Abb. 4.14 a) ein deutlich anderes Verhalten: Innerhalb der Oxidschicht beträgt die Feldstärke an der Grenzfläche zum Metall nahezu $1 \mathrm{~V} / \mathrm{nm}$ 


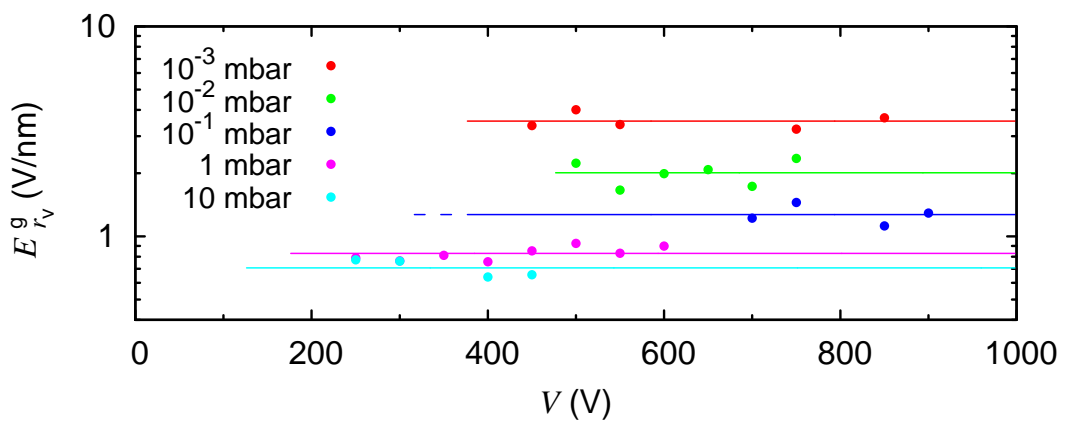

Abbildung 4.15: Abhängigkeit der Feldstärke an der Oxidoberfläche im Gasraum am Ort verschwindender Oxiddicke $E_{r_{\mathrm{v}}}^{\mathrm{g}}$ von der an der Spitze anliegenden Spannung $V$. Die horizontalen Linien sind Mittelwerte für den jeweiligen $\mathrm{H}_{2} \mathrm{O}$-Druck. Sie sind von derjenigen Spannung an dargestellt, bei der die Oxidationsreaktion einsetzt. Da die Spannung jeweils für $10 \mathrm{~s}$ an der Spitze anlag, ist nach 4.2.1 die Oxidationsreaktion für die einzelnen Oxidationsschritte lediglich bei einem Druck von 10 mbar $\mathrm{H}_{2} \mathrm{O}$ zum Erliegen gekommen. Für alle übrigen Druckwerte ist die stabile Spitzenmorphologie noch nicht vollständig erreicht.

und sinkt für große Entfernungen von der Grenzfläche deutlich ab. Direkt an der Oxidoberfläche im Gasraum wird die höchste Feldstärke am Ort verschwindender Oxiddicke $r_{\mathrm{v}}$ mit $3 \mathrm{~V} / \mathrm{nm}$ bis $4 \mathrm{~V} / \mathrm{nm}$ erreicht, zum Apex hin fällt die Feldstärke ab. Auch hier zeigt sich unabhängig von der an der Spitze anliegenden Spannung qualitativ das gleiche Verhalten.

Um nun die Abhängigkeit der Oxidationsreaktion vom elektrischen Feld übersichtlich beschreiben zu können, wird die Feldstärke an der Oxidoberfläche im Gasraum am Ort verschwindender Oxiddicke $E_{r_{\mathrm{V}}}^{\mathrm{g}}$ betrachtet (siehe Abb. 4.14). Da nach den Ergebnissen aus 4.2.1 für große Zeiten keine messbare Änderung von $r_{\mathrm{v}}$ beobachtet wird, liegt nahe, dass die Feldstärke an dieser Position zentral für den Ablauf der Oxidationsreaktion ist. Aufgrund der Probenmorphologie ist $E_{r_{\mathrm{V}}}^{\mathrm{g}}$ darüber hinaus recht stabil gegenüber einer möglichen Variation der Permittivitätszahl. Die Abhängigkeit der Größe $E_{r_{\mathrm{v}}}^{\mathrm{g}}$ von der für jeweils $10 \mathrm{~s}$ an der Spitze anliegenden Spannung $V$ bei unterschiedlichem $\mathrm{H}_{2} \mathrm{O}$-Druck zeigt Abb. 4.15. Im Rahmen der Messgenauigkeit nimmt $E_{r_{\mathrm{v}}}^{\mathrm{g}}$ bei gegebenem $\mathrm{H}_{2} \mathrm{O}$-Druck unabhängig von der Spannung $V$ einen konstanten Wert an. Die Oxidationsreaktion, in deren Verlauf durch die Umwandlung des Metalls in Oxid der Wert für $r_{\mathrm{v}}$ stetig ansteigt und $E_{r_{\mathrm{v}}}^{\mathrm{g}}$ entsprechend abfällt, läuft folglich stets bis zum Erreichen eines festen Wertes $E_{r_{\mathrm{v}}}^{\mathrm{g}} \mathrm{ab}$.

\subsubsection{Feldstärkeverteilung in Abhängigkeit vom $\mathrm{H}_{2} \mathrm{O}$-Druck}

Die Feldstärkeverteilung in der Umgebung der nanoskaligen Spitzen in Abhängigkeit vom $\mathrm{H}_{2} \mathrm{O}$-Druck zeigt Abb. 4.16. Für die Berechnung der Feldstärke mit der FiniteElemente-Methode wurde dabei die in Abb. 4.6 dargestellte Spitzenmorphologie zugrunde gelegt. Somit liegt für fast alle Druckwerte bereits die stabile Morphologie vor, lediglich im Bereich von $10^{-2}$ mbar bis 1 mbar $\mathrm{H}_{2} \mathrm{O}$ erfolgen noch Änderungen. Innerhalb der Oxidschicht liegt die Feldstärke bei allen Druckwerten unterhalb von $1 \mathrm{~V} / \mathrm{nm}$, allein an der Grenzfläche zum Metall nahe der Symmetrieachse wird dieser Wert bei denjenigen Spitzen überschritten, bei denen die Metall-Oxid-Grenzfläche konvex in Bezug auf 


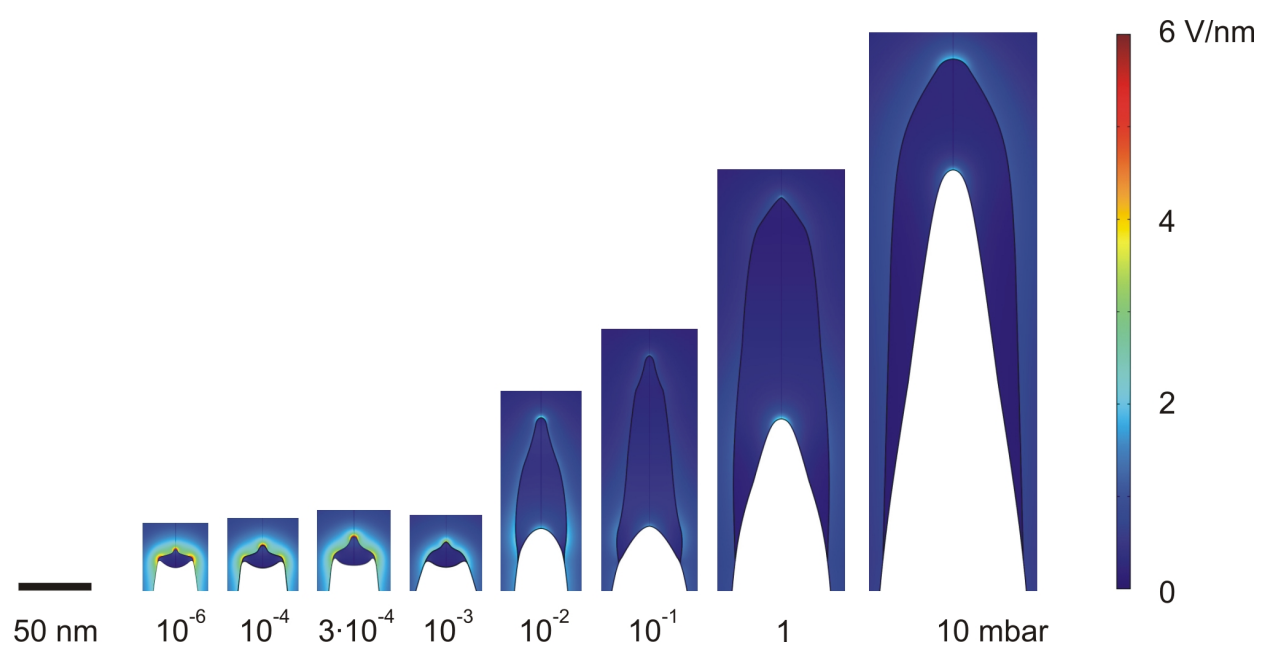

Abbildung 4.16: Mit der Finite-Elemente-Methode berechnete Feldstärkeverteilung in der Umgebung verschiedener W-Spitzen, bei denen die Oxidationreaktion bei dem jeweils angegebenen $\mathrm{H}_{2} \mathrm{O}$-Druck für 2187 s erfolgte. Der Betrag der elektrischen Feldstärke ist durch die angegebene Farbskala kodiert.

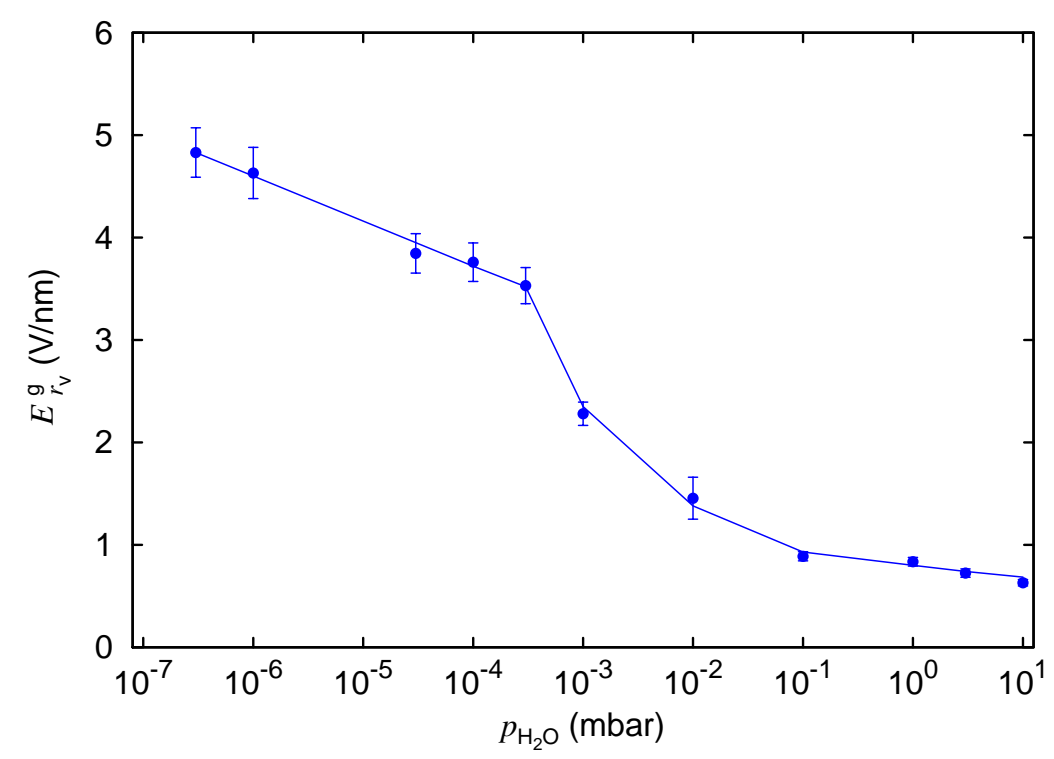

Abbildung 4.17: Auftragung der Feldstärke an der Oxidoberfläche im Gasraum am Ort verschwindender Oxiddicke $E_{r_{\mathrm{v}}}^{g}$ in Abhängigkeit vom $\mathrm{H}_{2} \mathrm{O}$-Druck. Die Werte für $E_{r_{\mathrm{v}}}^{\mathrm{g}}$ wurden mit der FiniteElemente-Methode für Oxidationszeiten von $729 \mathrm{~s}$ und $2187 \mathrm{~s}$ berechnet. Da nach 4.2 .1 die Reaktion im Druckbereich von $10^{-2}$ mbar bis 1 mbar $\mathrm{H}_{2} \mathrm{O}$ noch nicht vollständig zum Erliegen gekommen ist, dürften die $E_{r_{v}}^{g}$-Werte für die stabile Morphologie etwas geringer als die angegebenen sein.

die metallische Spitze ist. Direkt an der Oxidoberfläche im Gasraum hingegen wird mit abnehmendem $\mathrm{H}_{2} \mathrm{O}$-Druck ein Anstieg der Feldstärke auf bis zu $6 \mathrm{~V} / \mathrm{nm}$ beobachtet.

Da nach 4.4.1 die Oxidationsreaktion bis zum Erreichen einer konstanten Feldstärke $E_{r_{\mathrm{v}}}^{\mathrm{g}}$ abläuft, wird diese Größe auch herangezogen, um die Abhängigkeit des für die Oxidationsreaktion notwendigen elektrischen Feldes vom $\mathrm{H}_{2} \mathrm{O}$-Druck zu quantifizieren. Eine Auftragung von $E_{r_{\mathrm{v}}}^{\mathrm{g}}$ als Funktion des Druckes zeigt Abb. 4.17. Das für den Ablauf der Oxidationsreaktion notwendige elektrische Feld weist eine deutliche Druckabhängigkeit mit einem monotonen Abfall bei steigendem $\mathrm{H}_{2} \mathrm{O}$-Druck auf. Für Druckwerte unterhalb 
von $10^{-3}$ mbar wird ein lineares Verhalten der Form

$$
E_{r_{\mathrm{v}}}^{\mathrm{g}}\left(p_{\mathrm{H}_{2} \mathrm{O}}\right)=\left[-0,19(1) \cdot \ln \left(p_{\mathrm{H}_{2} \mathrm{O}} / \mathrm{p}^{0}\right)+0,7(2)\right] \mathrm{V} / \mathrm{nm}
$$

gefunden. Bei einem Druck von $10^{-3}$ mbar schließst sich ein zweites Regime an, welches durch eine komplexere Druckabhängigkeit von $E_{r_{\mathrm{v}}}^{\mathrm{g}}$ gekennzeichnet ist. Der Übergang zum zweiten Regimen geht dabei mit einem starken Abfall von $E_{r_{\mathrm{v}}}^{\mathrm{g}}$ einher. 


\section{Resultate bei Variation des Spitzenmaterials und der Gasspezies}

Nach der Darstellung des feldinduzierten Oxidationsverhaltens nanoskaliger Spitzen aus Wolfram in Kapitel 4 werden hier zunächst Resultate bei Verwendung anderer Spitzenmaterialien vorgestellt. Bei Aluminium und Silizium wird dabei ein qualitativ ähnliches Verhalten wie bei Wolfram gefunden, während an Nickel und Silber ein abweichendes Verhalten beobachtet wird. Weiterhin werden die Ergebnisse von Oxidationsexperimenten unter Verwendung unterschiedlicher sauerstoffenthaltender Gase vorgestellt.

\subsection{Al-Spitzen}

Zur Veranschaulichung des Oxidationsverhaltens von Aluminium zeigt Abb. 5.1 eine Folge von TEM-Aufnahmen einer nanoskaligen Spitze, an die unter Umgebungsatmosphäre für jeweils $81 \mathrm{~s}$ die angegebenen Spannungen angelegt wurden. Bereits vor Anlegen einer Spannung $(0 \mathrm{~V})$ ist auf der einkristallinen Spitze eine durch die elektrochemische Präparation entstandene Oxidschicht von $4 \mathrm{~nm}$ Dicke vorhanden. Nach Anlegen einer Spannung von $1000 \mathrm{~V}$ weist die Spitze im Apexbereich eine neu gebildete Oxidschicht auf, deren Dicke von $28 \mathrm{~nm}$ auf der Symmetrieachse mit zunehmendem Abstand vom Apex abnimmt bis sie schließlich nicht mehr auflösbar ist. Bei stufenweiser Erhöhung

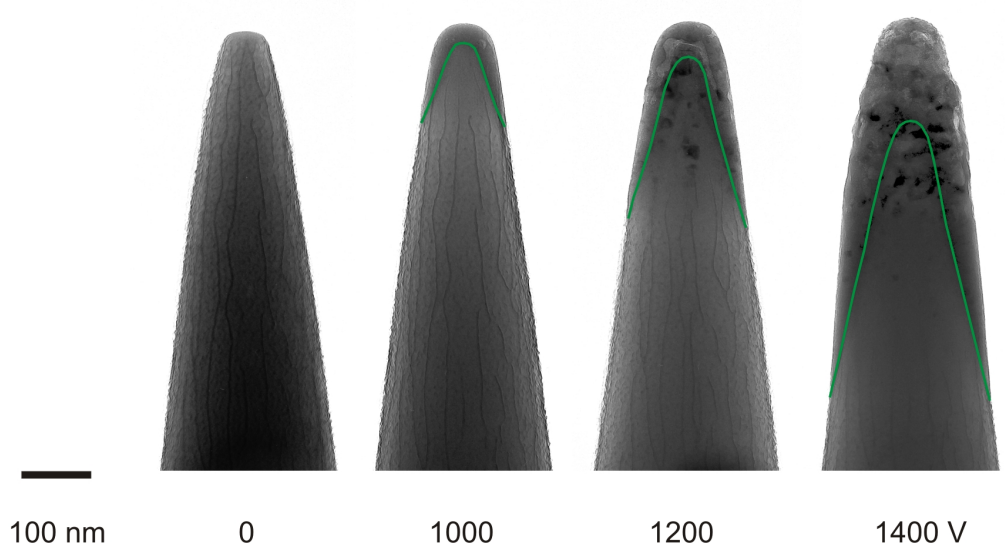

Abbildung 5.1: Folge von TEM-Aufnahmen einer Al-Spitze, an die für jeweils $81 \mathrm{~s}$ an Umgebungsatmosphäre bei Standarddruck die angegebenen Spannungen angelegt wurden. Die Grenzfläche zwischen Metall und Oxid ist durch die grüne Linie hervorgehoben. Die Spitze wurde zuvor im Feldionenmikroskop auf $5 \mathrm{kV}$ entwickelt. 


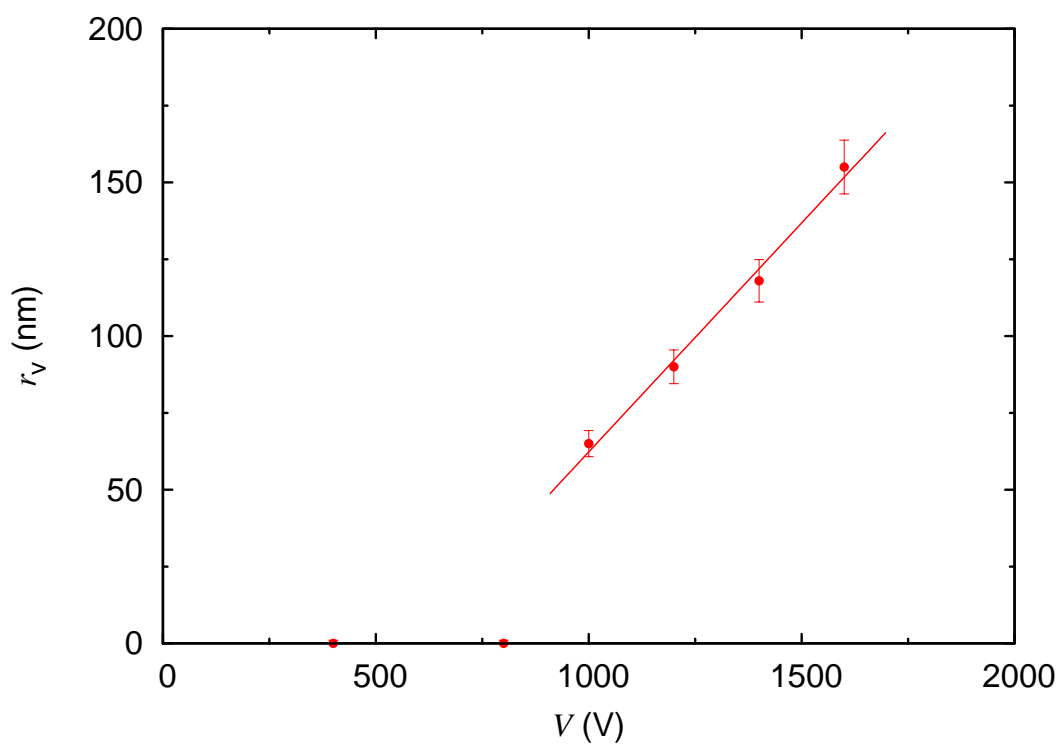

Abbildung 5.2: Radius der Spitze am Ort verschwindender Oxiddicke $r_{\mathrm{v}}$ in Abhängigkeit der Spannung $V$ für die in Abb. 5.1 dargestellte Al-Spitze.

der Spannung wächst die Oxidschicht, die Morphologie von Spitze und Oxidschicht bleibt jedoch qualitativ nahezu unverändert. Wird als Maß für den Grad der Oxidationsreaktion der Radius der Spitze am Ort verschwindender Oxiddicke $r_{\mathrm{v}}$ gegen die angelegte Spannung $V$ aufgetragen, ${ }^{1}$ so zeigt sich das in Abb. 5.2 dargestellte Verhalten. Für Spannungen bis $800 \mathrm{~V}$ wird keine Oxidbildung auf der Al-Spitze beobachtet $\left(r_{\mathrm{v}}=0\right)$. Beträgt die angelegte Spannung jedoch mindestens $1000 \mathrm{~V}$, so bildet sich eine Oxidschicht auf der Al-Spitze, wobei $r_{\mathrm{v}}$ mit zunehmender Spannung monoton ansteigt.

Das feldinduzierte Oxidationsverhalten von Al-Spitzen unter Umgebungsatmosphäre ähnelt somit stark dem in 4.1 beschriebenen Oxidationsverhalten von W-Spitzen unter $\mathrm{H}_{2} \mathrm{O}$-Atmosphäre: In beiden Fällen ist eine Mindestfeldstärke für den Ablauf der Oxidationsreaktion erforderlich und es wird ein linearer Anstieg von $r_{\mathrm{v}}$ mit der Spannung $V$ beobachtet. Weiterhin ist die Morphologie der auf den Al-Spitzen gebildeten Oxidschichten nahezu identisch mit derjenigen, die sich bei Oxidation von W-Spitzen unter einem $\mathrm{H}_{2} \mathrm{O}$-Druck von 10 mbar einstellt. Dies erscheint plausibel, da bei einer üblichen relativen Luftfeuchtigkeit der Partialdruck von $\mathrm{H}_{2} \mathrm{O}$ in der Umgebungsatmosphäre ebenfalls in der Größenordnung von 10 mbar liegt. Um zu klären, inwieweit die Oxidationsreaktion an Al-Spitzen in gleicher Weise wie an W-Spitzen erfolgt, werden im Folgenden Resultate zur Oxidation von Al-Spitzen unter reiner $\mathrm{H}_{2} \mathrm{O}$-Atmosphäre definierten Druckes vorgestellt.

\footnotetext{
${ }^{1}$ Die Position $r_{\mathrm{v}}$ bezeichnet wiederum den Radius, an dem die Dicke des unter dem Einfluss des elektrischen Feldes gebildeten Oxides verschwindet (siehe Abb.4.1). Das natürliche Oxid auf der Spitzenoberfläche bleibt dabei unberücksichtigt.
} 

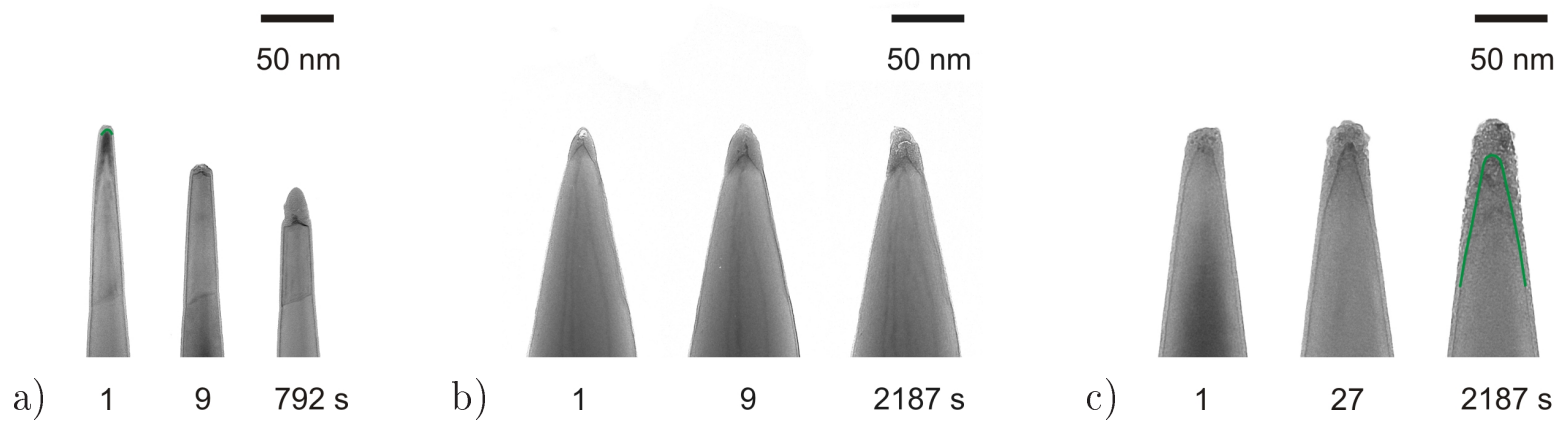

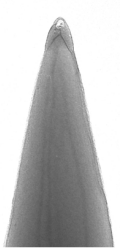

b)

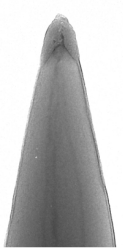

9

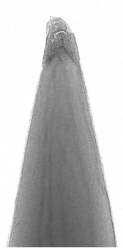

$2187 \mathrm{~s}$

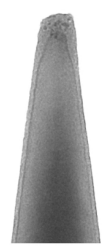

c) 1

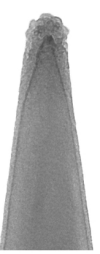

27

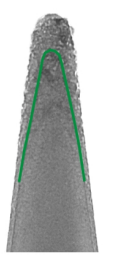

$2187 \mathrm{~s}$

Abbildung 5.3: Folge von TEM-Aufnahmen dreier Al-Spitzen, nachdem bei einem Druck von a) $10^{-4}$ mbar, b) $10^{-1}$ mbar und c) 10 mbar $\mathrm{H}_{2} \mathrm{O}$ eine Spannung von $350 \mathrm{~V}$ für die jeweils angegebene Zeit angelegt wurde. ${ }^{2}$

\subsubsection{Kinetik der Morphologieentwicklung}

Die zeitabhängige Entwicklung der Morphologie dreier Spitzen, bei denen die Oxidationsreaktion unter verschiedenem $\mathrm{H}_{2} \mathrm{O}$-Druck erfolgte, ist in Abb. 5.3 dargestellt. Bei einem Druck von 10 mbar $\mathrm{H}_{2} \mathrm{O}$ wird bereits nach $1 \mathrm{~s}$ die Ausbildung einer Oxidschicht auf der Spitze beobachtet. Für längere Zeiten nehmen $r_{\mathrm{v}}$ und die Dicke der Oxidschicht auf der Symmetrieachse $d$ monoton zu. Beträgt der $\mathrm{H}_{2} \mathrm{O}$-Druck $10^{-1}$ mbar, so bildet sich ebenfalls innerhalb von 1 s eine Oxidschicht, welche für größere Zeiten weiter wächst. Im Gegensatz zum Verhalten bei einem $\mathrm{H}_{2} \mathrm{O}$-Druck von 10 mbar ähnelt die Metall-OxidGrenzfläche jedoch nicht mehr der Spitzenoberfläche vor Beginn der Oxidationsreaktion, sondern nimmt eine kegelförmige Gestalt an. Bei einem nochmals geringeren Druck von $10^{-4}$ mbar $\mathrm{H}_{2} \mathrm{O}$ zeigt sich ein qualitativ ähnliches Verhalten wie bei $10^{-1} \mathrm{mbar}$, die Werte von $r_{\mathrm{v}}$ sind allerdings geringer.

Qualitativ zeigt die Morphologie der Al-Spitzen somit eine ähnliche Zeit- und Druckabhängigkeit wie die der W-Spitzen. Bei Aluminium wird jedoch nicht die Ausbildung von Oxidspitzen beobachtet, und der bei abnehmendem $\mathrm{H}_{2} \mathrm{O}$-Druck erfolgende Übergang der Krümmung der Metall-Oxid-Grenzfläche von konvex zu konkav in Bezug auf die metallische Spitze ist schwächer ausgeprägt. Zur quantitativen Erfassung der Kinetik der Oxidationsreaktion ist in Abb. 5.4 der aus den TEM-Aufnahmen bestimmte Wert für $r_{\mathrm{v}}$ gegen die Zeit aufgetragen. Unabhängig vom $\mathrm{H}_{2} \mathrm{O}$-Druck wird ein monotoner Anstieg von $r_{\mathrm{v}}$ mit der Zeit beobachtet, wobei die Werte von $r_{\mathrm{v}}$ stets deutlich unterhalb der bei Wolfram gemessenen liegen. Für Druckwerte von höchstens $10^{-1}$ mbar $\mathrm{H}_{2} \mathrm{O}$ kommt die Oxidationsreaktion offenbar nach $10^{2} \mathrm{~s}$ zum Erliegen. Bei höheren Drücken wird hingegen auch nach $10^{3} \mathrm{~s}$ noch eine Zunahme von $r_{\mathrm{v}}$ beobachtet. Auch der in Abb. 5.5 dargestellte zeitliche Verlauf der Dicke der Oxidschicht auf der Symmetrieachse $d$ deutet darauf hin, dass die Oxidationsreaktion bei Drücken von höchstens $10^{-1}$ mbar $\mathrm{H}_{2} \mathrm{O}$ für lange Zeiten zum Erliegen kommt. Im Gegensatz zum Wolfram läuft bei Aluminium jedoch

\footnotetext{
${ }^{2}$ In sämtlichen Messungen bei einem $\mathrm{H}_{2} \mathrm{O}$-Druck von weniger als 1 mbar wurde eine Spannung von $700 \mathrm{~V}$ an die Al-Spitzen angelegt, um noch Oxidschichten hinreichender Größe zu erhalten. Zur Gewährleistung der Vergleichbarkeit mit den übrigen Daten wurden wie bei Wolfram die abgebildeten TEM-Aufnahmen dieser Spitzen sowie die daraus bestimmten Werte $r_{\mathrm{v}}$ und $d$ je mit einem Faktor $\frac{1}{2}$ skaliert. Die Berechnungen mit der Finite-Elemente-Methode erfolgten hingegen unter Berücksichtigung der tatsächlichen Größen- und Spannungsverhältnisse.
} 


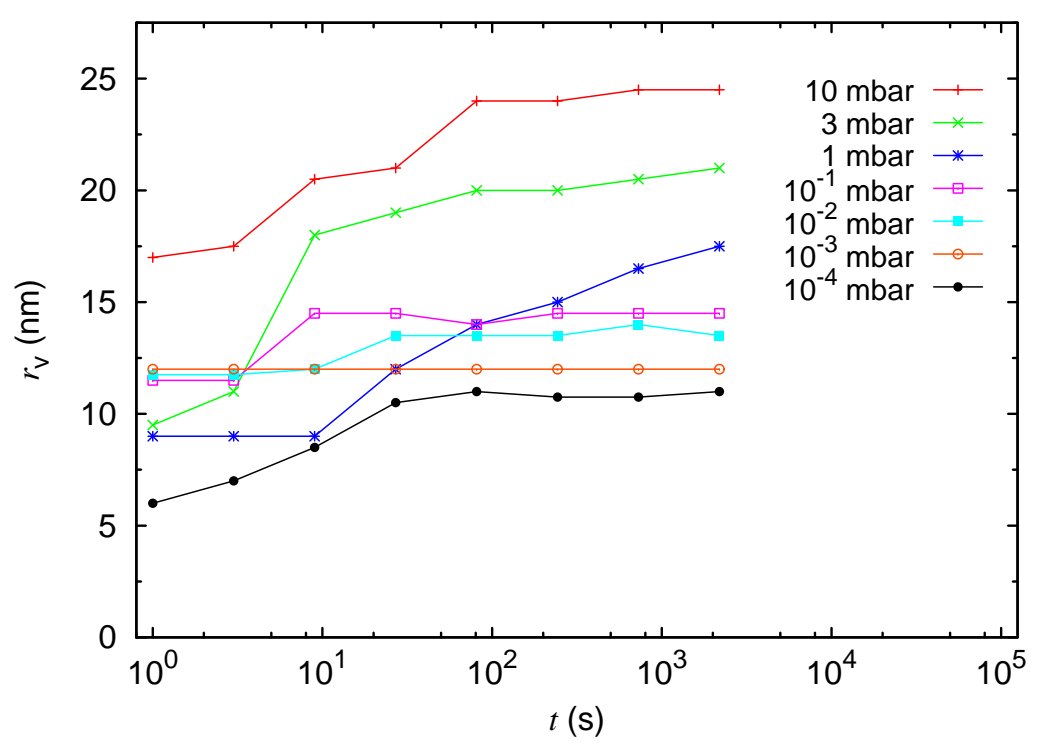

Abbildung 5.4: Zeitlicher Verlauf des aus TEM-Aufnahmen bestimmten Wertes $r_{\mathrm{v}}$ für Messreihen zur Oxidation von Al-Spitzen bei verschiedenem $\mathrm{H}_{2} \mathrm{O}$-Druck. An den Spitzen lag je eine Spannung von $350 \mathrm{~V}$ an. ${ }^{2}$ Die Genauigkeit der Werte liegt bei etwa $10 \%$, aus Gründen der Übersichtlichkeit sind jedoch keine Fehler angegeben.

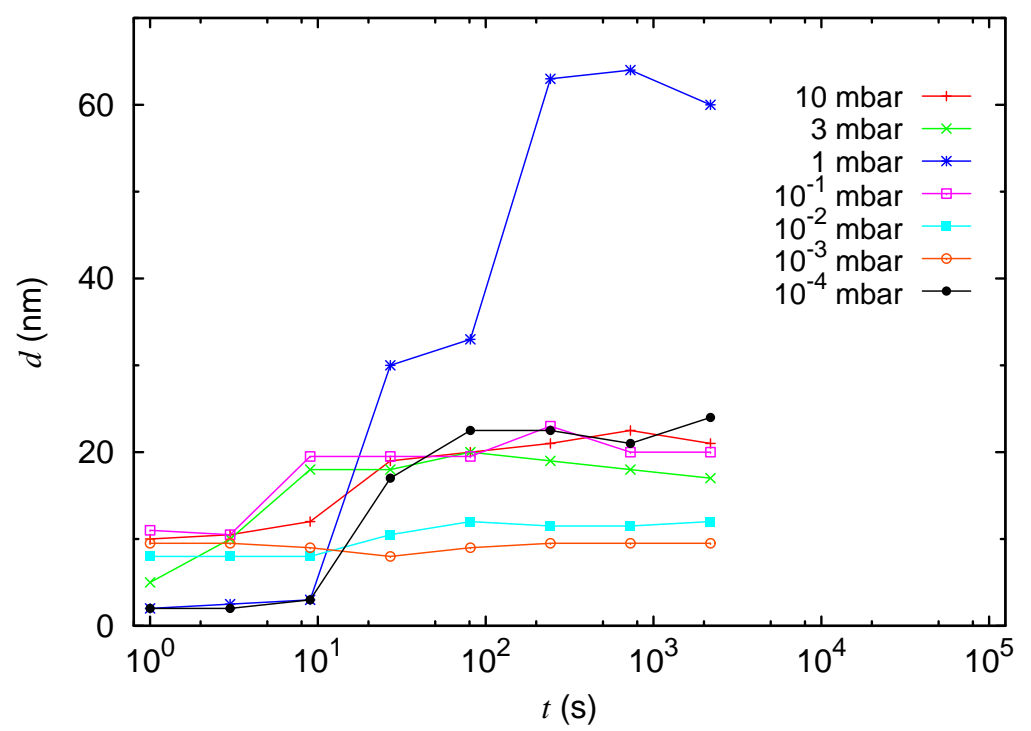

Abbildung 5.5: Zeitlicher Verlauf des aus TEM-Aufnahmen bestimmten Wertes $d$ für Messreihen zur Oxidation von Al-Spitzen bei verschiedenem $\mathrm{H}_{2} \mathrm{O}$-Druck. Für weitere Angaben siehe Abb. 5.4.

auf der untersuchten Zeitskala offenbar kein Prozess ab, der eine drastische Verringerung von $d$ zur Folge hat. Die Kinetik der Morphologieentwicklung bei der Oxidation von Aluminium weist somit für kurze Zeiten durchaus Unterschiede zu dem bei Wolfram beobachteten Verhalten auf. Für Zeiten in der Größenordnung von $10^{3} \mathrm{~s}$ verhalten sich beide Elemente jedoch ähnlich. 


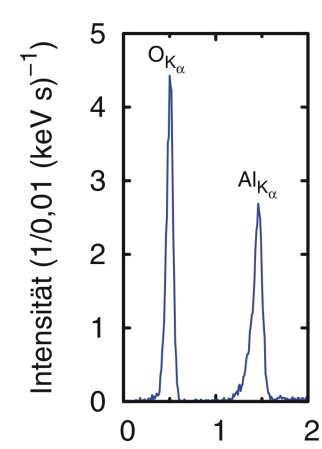

a) b) $100 \mathrm{~nm}$

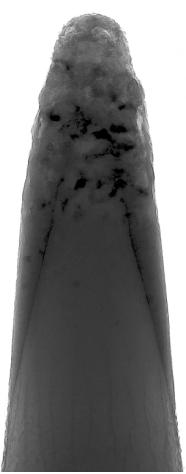

c)

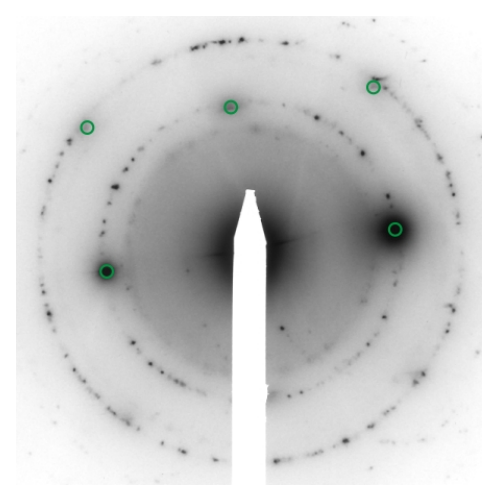

Abbildung 5.6: a) EDX-Spektrum der in der TEM-Aufnahme in b) abgebildeten Oxidschicht mit charakteristischen Linien für Sauerstoff und Aluminium. c) Feinbereichs-Beugungsbild der in b) abgebildeten Spitze. Mit grünen Kreisen markiert ist das Beugungsbild der einkristallinen Al-Spitze, die in geringster Entfernung zum Nullstrahl liegenden Reflexe sind vom Typ \{200\}. An die Aluminiumspitze wurde zuvor an Umgebungsatmosphäre bei Umgebungsdruck für $81 \mathrm{~s}$ eine Spannung von $1400 \mathrm{~V}$ angelegt.

\subsubsection{Struktur und Stöchiometrie des Oxides}

Zur chemischen Charakterisierung der auf den Al-Spitzen gebildeten Oxidschichten wurde zunächst wiederum EDX eingesetzt, ein typisches Spektrum zeigt Abb. 5.6 a). Die neben der K-Linie für Aluminium mit hoher Intensität auftretende K-Linie für Sauerstoff weist auf das Vorliegen eines Oxides hin. Die Bestimmung der Stöchiometrie des Oxides erfolgt mit Laser-unterstützter Atomsondentomographie. Dabei treten in den Massenspektren deutliche Peaks für $\mathrm{Al}_{\mathrm{x}} \mathrm{O}_{\mathrm{y}}$ sowie für $\mathrm{H}_{2} \mathrm{O}$ und $\mathrm{OH}$ auf. Für eine Al-Spitze, bei der die Oxidationsreaktion bei einem Druck von 3 mbar $\mathrm{H}_{2} \mathrm{O}$ stattfand, konnte so die Sauerstoffkonzentration in der Oxidschicht zu 57(3) At.\% O bestimmt werden. Wie bei Wolfram liegt somit auch bei Aluminium die ermittelte Sauerstoffkonzentration nahe bei der für stöchiometrisches $\mathrm{Al}_{2} \mathrm{O}_{3}$ erwarteten.

Die Bestimmung der Struktur der Oxidschichten erfolgt mittels Feinbereichsbeugung. Abb. 5.6c) zeigt ein typisches Beugungsbild wie es auch für Spitzen, bei denen die Oxidationsreaktion an reiner $\mathrm{H}_{2} \mathrm{O}$-Atmosphäre erfolgt, gefunden wird. Da die Reflexe der einkristallinen Al-Spitze nicht auf den Beugungsringen liegen, werden die Beugungsringe nicht durch polykristallines Aluminium, sondern durch das Oxid hervorgerufen. Die Körnigkeit der Beugungsringe und der im Hellfeldbild der Spitze deutlich erkennbare Kontrast innerhalb der Oxidschicht weisen auf Oxidkristallite mit einer Größe im Bereich von $10 \mathrm{~nm}$ hin. Somit liegen in den Aluminiumoxidschichten deutlich größere Kristallite als in den Wolframoxidschichten vor.

\subsubsection{Feldstärkeverteilung in Abhängigkeit vom $\mathrm{H}_{2} \mathrm{O}$-Druck}

Zur Berechnung der Feldstärkeverteilung in der Umgebung der Al-Spitzen wurde für die Oxidschichten eine Permittivitätszahl $\varepsilon_{\mathrm{r}}=10$ entsprechend den in [Bire1977, Prat1969] veröffentlichten Werten für $\varepsilon_{\mathrm{r}}$ zugrunde gelegt. Die elektrische Eigenschaft der Aluminiumoxidschicht wird somit durch denselben Wert wie die der Wolframoxidschichten charakterisiert. Daher folgt aus der Ähnlichkeit der Spitzenmorphologie für beide Ele- 


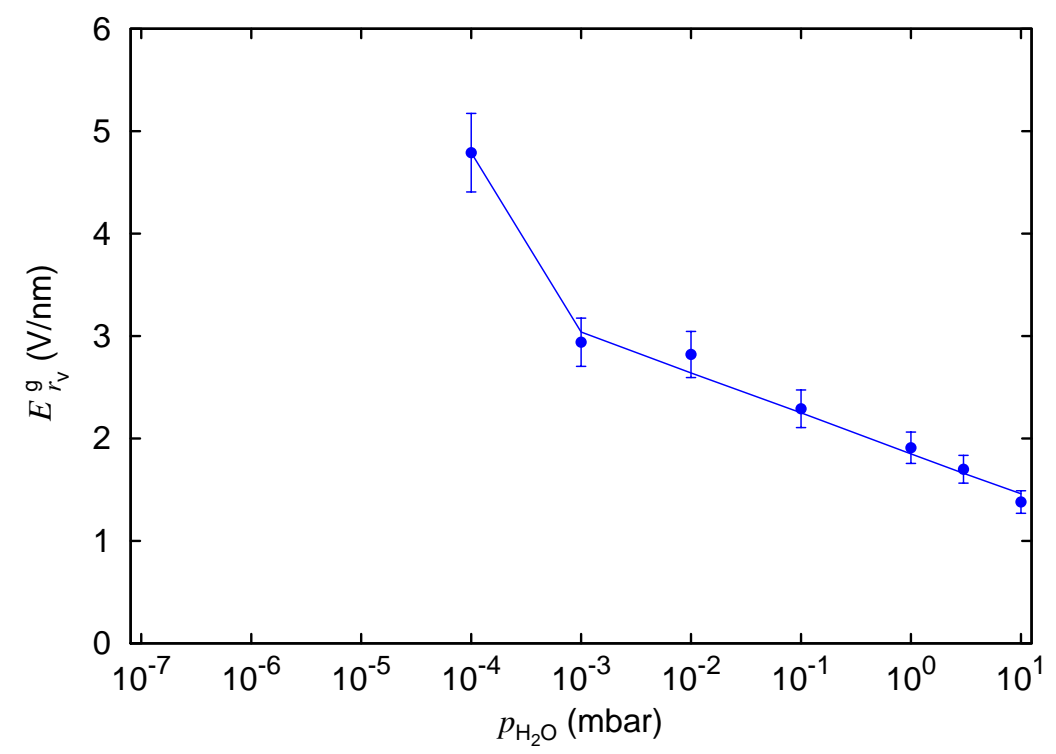

Abbildung 5.7: Auftragung der Feldstärke an der Oxidoberfläche im Gasraum am Ort verschwindender Oxiddicke $E_{r_{\mathrm{v}}}^{\mathrm{g}}$ in Abhängigkeit vom $\mathrm{H}_{2} \mathrm{O}$-Druck. Die Werte für $E_{r_{\mathrm{v}}}^{\mathrm{g}}$ wurden mit der Finite-ElementeMethode für Oxidationszeiten der Al-Spitzen von $2187 \mathrm{~s}$ berechnet. Sofern für einen Druck oberhalb von $10^{-1}$ mbar Kondensation von $\mathrm{H}_{2} \mathrm{O}$ auf der Spitze erfolgt (siehe 6.2), ist zu erwarten, dass die Berechung von $E_{r_{\mathrm{v}}}^{\mathrm{g}}$ wegen der Vernachlässigung der von 1 verschiedenen Permittivität des $\mathrm{H}_{2} \mathrm{O}$ zu hohe Werte liefert. Die tatsächlich vorliegende Feldstärke an der Oxidoberfläche wäre demnach geringer als angegeben.

mente auch eine qualitativ ähnliche Feldverteilung in der Spitzenumgebung. Das für die Oxidationsreaktion notwendige elektrische Feld wird wiederum durch die Feldstärke an der Oxidoberfläche am Ort verschwindender Oxiddicke $E_{r_{\mathrm{v}}}^{\mathrm{g}}$ quantifiziert. Eine Auftragung von $E_{r_{\mathrm{v}}}^{\mathrm{g}}$ in Abhängigkeit vom $\mathrm{H}_{2} \mathrm{O}$-Druck zeigt Abb. 5.7. Die Feldstärke nimmt mit zunehmendem Druck monoton ab, wobei bei Überschreiten eines Druckes von $10^{-4}$ mbar $\mathrm{H}_{2} \mathrm{O}$ ein deutlicher Abfall von $E_{r_{\mathrm{v}}}^{\mathrm{g}}$ zu verzeichnen ist. Qualitativ wird somit das gleiche Verhalten wie bei Wolfram beobachtet. Bei den Al-Spitzen liegen die Werte von $E_{r_{\mathrm{v}}}^{\mathrm{g}}$ jedoch durchweg höher.

\subsection{Si-Spitzen}

Die sich bei der Oxidation von Si-Spitzen an Umgebungsatmosphäre einstellende Spitzenmorphologie zeigt Abb. 5.8. Qualitativ wird das gleiche Verhalten wie bei der Oxidation von Al-Spitzen an Umgebungsatmosphäre beobachtet. Im Apexbereich der Spitze bildet sich eine Oxidschicht, deren Dicke mit zunehmendem Abstand vom Apex abnimmt. Die Auftragung des Radius der Spitze am Ort verschwindender Oxiddicke $r_{\mathrm{v}}$ in Abhängigkeit von der Spannung $V$ in Abb. 5.9 zeigt, dass wie bei Wolfram und Aluminium eine Mindestspannung an der Spitze anliegen muss, damit eine Oxidbildung erfolgt. Oberhalb dieser Mindestspannung wird wiederum ein linearer Anstieg von $r_{\mathrm{v}}$ mit der Spannung beobachtet. Der Effekt der feldinduzierten Oxidation ist somit nicht auf Metalle beschränkt, sondern kann auch bei Halbleitern beobachtet werden. 


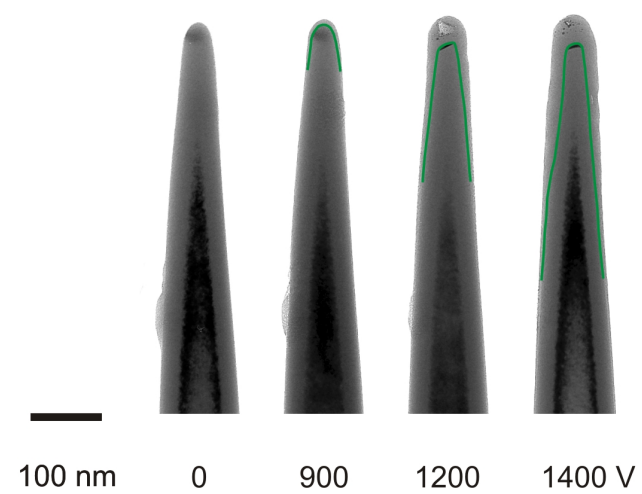

Abbildung 5.8: Folge von TEM-Aufnahmen einer Si-Spitze, an die für jeweils $10 \mathrm{~s}$ an Umgebungsatmosphäre bei Standarddruck die angegebenen Spannungen angelegt wurden. Die Grenzfläche zwischen Silizium und Oxid ist aufgrund der geringeren Absorption im Oxid erkennbar, sie ist durch die grüne Linie hervorgehoben. Durch die Bestrahlung der Spitze mit $30 \mathrm{keV}$ Ga-Ionen während der Präparation mittels FIB liegt das Silizium an der Oberfläche in einer etwa $30 \mathrm{~nm}$ dicken Schicht nicht mehr in einkristalliner Form vor. Die starke Absorption im Apexbereich der Spitze ist auf eine Implantation von Platin oder Gallium während der FIB-Präparation zurückzuführen.

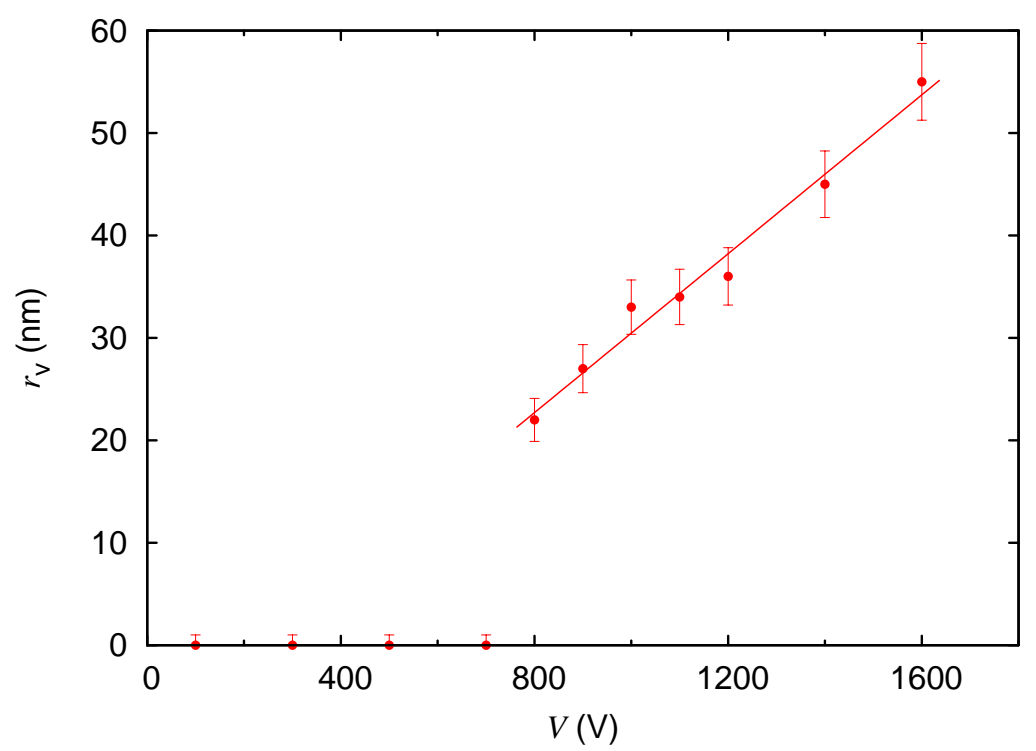

Abbildung 5.9: Radius der Spitze am Ort verschwindender Oxiddicke $r_{\mathrm{v}}$ in Abhängigkeit der Spannung $V$ für die in Abb.5.8 dargestellte Si-Spitze mit einem anfänglichen Krümmungsradius am Apex von $15 \mathrm{~nm}$.

\subsection{Weitere Spitzenmaterialien}

Neben den bereits beschriebenen Untersuchungen an Wolfram, Aluminium und Silizium wurden weitere Elemente auf ihr Oxidationsverhalten unter dem Einfluss eines externen elektrischen Feldes hin untersucht. Sämtliche Experimente fanden dabei an Umgebungsatmosphäre statt.

An Spitzen aus Molybdän wird die Entstehung der gleichen Morphologie wie bei Aluminium, Silizium und Wolfram bei einem Druck in der Größenordnung von 10 mbar $\mathrm{H}_{2} \mathrm{O}$ beobachtet. EDX Analysen zeigen deutliche Peaks für Sauerstoff und Molybdän 
und bestätigen somit die Oxidbildung.

Bei Nickel konnte keine feldinduzierte Ausbildung von Oxidschichten beobachtet werden. Wurde in dem Oxidationsexperiment die an der Ni-Spitze anliegende Spannung schrittweise erhöht, so verringerte sich oberhalb einer gewissen Spannung die Länge der Spitze und somit auch die Krümmung der Spitze am Apex. Es wurde jedoch stets wie auch vor dem Anlegen einer Spannung nur eine $5 \mathrm{~nm}$ dicke Oxidschicht auf der Spitze gefunden. Der Verlust von Spitzenmaterial deutet auf den Einsatz der Feldverdampfung hin, wobei entweder Nickel oder aber unter dem Einfluss des Feldes gebildetes Nickeloxid verdampft.

Spitzen aus Silber zeigen unter dem Einfluss eines Feldes eine Morphologieänderung ähnlich wie Aluminium und Silizium. Die sich bildenden Schichten weisen jedoch eine deutlich irregulärere Form mit einer sehr großen Rauigkeit der Oxidoberfläche auf. Strukturell sind die Schichten offenbar inhomogen: Während die Gesamtheit der Schicht bei Feinbereichsbeugung ein polykristallines Beugungsbild aufweist, zeigen einzelne Bereiche der Schicht im Hellfeldbild keinerlei Orientierungskontrast und erscheinen somit im TEM amorph. Die chemische Charakterisierung der Schichten mit EDX bestätigt wegen des Auftretens von Peaks für Sauerstoff und Silber die Oxidbildung.

\subsection{Variation der Gasatmosphäre}

Bei sämtlichen bislang geschilderten Resultaten erfolgte die Oxidationsreaktion an reiner $\mathrm{H}_{2} \mathrm{O}$-Atmosphäre oder an Umgebungsatmosphäre. Dabei wird bei Experimenten an Umgebungsatmosphäre stets die gleiche Spitzenmorphologie gefunden wie sie bei Experimenten an reiner $\mathrm{H}_{2} \mathrm{O}$-Atmosphäre auftritt, sofern der $\mathrm{H}_{2} \mathrm{O}$-Druck mit 10 mbar etwa dem Partialdruck von $\mathrm{H}_{2} \mathrm{O}$ in der Umgebungsatmosphäre entspricht. Um den Einfluss der sauerstoffenthaltenden Spezies auf die feldinduzierte Oxidationsreaktion zu klären, wurde ergänzend das Oxidationsverhalten von W-Spitzen unter reiner Sauerstoffatmosphäre untersucht. Bei $\mathrm{O}_{2}$-Druckwerten in der Größenordnung von $10^{2}$ mbar - entsprechend dem Partialdruck von $\mathrm{O}_{2}$ an Umgebungsatmosphäre - wurde dabei keine Ausbildung von Oxidschichten mit einer Morphologie wie bei der Reaktion an Umgebungsatmosphäre beobachtet. Die Form, in der Sauerstoff für die Oxidationsreaktion zur Verfügung steht, spielt somit eine entscheidende Rolle für die feldinduzierte Oxidation. Eine eingehendere Diskussion dieses Befundes erfolgt in 6.4.2. 


\section{Diskussion}

In diesem Kapitel werden die Resultate der Untersuchungen zur feldinduzierten Oxidation nanoskaliger Spitzen diskutiert. Grundlage sind dabei vornehmlich die in Kapitel 4 vorgestellten Ergebnisse für W-Spitzen.

Zunächst wird diskutiert, welche Prozesse die Kinetik der Oxidationsreaktion bestimmen. Aus dem Zusammenwirken der einzelnen Teilreaktionen lässt sich dann eine Modell für die Entwicklung der Spitzenmorphologie ableiten. Im Anschluss wird der Einfluss des elektrischen Feldes auf die Oxidationsreaktion betrachtet. Dabei liegt der Fokus zum einen auf dem Aggregatzustand des $\mathrm{H}_{2} \mathrm{O}$ auf der Spitze, da sich das elektrische Feld stark auf den effektiven Partialdruck von $\mathrm{H}_{2} \mathrm{O}$ auswirkt. Zum anderen wird der Einfluss des Feldes auf den Ablauf der Oxidationsreaktion betrachtet. Dabei kann abgeleitet werden, dass die Reaktion von einem gewissen Stadium an kinetisch gehemmt ist. Der geschwindigkeitsbestimmende Schritt ist eine Reaktion von Hydroxidionen an der Oxidoberfläche. Ein Vergleich der Resultate für die unterschiedlichen Spitzenmaterialien zeigt schließlich, wie sich einzelne Materialeigenschaften auf den Prozess der feldinduzierten Tieftemperaturoxidation auswirken.

\subsection{Kinetik und Morphologieentwicklung}

In diesem Abschnitt wird diskutiert, welche physikalischen Prozesse die Kinetik der Oxidationsreaktion bestimmen. Grundlage sind dabei die in 4.2 vorgestellten Resultate der Kinetikmessungen an W-Spitzen. Als geschwindigkeitsbestimmender Schritt für das Anfangsstadium der Oxidationsreaktion bei geringen Druckwerten kann der Antransport von $\mathrm{H}_{2} \mathrm{O}$ identifiziert werden. Darüber hinaus wird diskutiert, wie die zeitabhängige Entwicklung der Spitzenmorphologie aus einer komplexen Wechselwirkung mit weiteren Prozessen folgt - insbesondere Volumendiffusion, Oberflächendiffusion und Feldverdampfung sind dabei relevant. Eine eingehendere Betrachtung der Morphologie des stabilen Zustandes am Ende diese Abschnittes bestätigt dabei, dass Feldverdampfung in erheblichem Maße stattfindet.

Zunächst wird jedoch diskutiert, ob die Morphologieentwicklung der W-Spitzen und der Reaktionsstrom gemäß Abb. 4.7 die gleiche Zeitabhängigkeit aufweisen. Dazu wird aus Abb. 4.4 die Zeit $t_{r_{\mathrm{v}}}$ bestimmt, die vergeht, bis $r_{\mathrm{v}}$ einen festen Wert annimmt, die Morphologieentwicklung der Spitze in Bezug auf $r_{\mathrm{v}}$ also zum Erliegen kommt. Aus Abb. 4.7 wird die Zeit $t_{\mathrm{s}}$ ermittelt, für die während der Oxidationsreaktion ein nahezu konstanter Strom messbar ist. Eine doppelt logarithmische Auftragung dieser beiden Zeiten in Abhängigkeit von $\mathrm{H}_{2} \mathrm{O}$-Druck zeigt Abb.6.1. Da $t_{\mathrm{s}}$ und $t_{r_{\mathrm{v}}}$ dieselbe Druckabhängigkeit aufweisen, gehen die Morphologieänderung der Spitze und der Stromfluss offenbar miteinander einher. Zur Untersuchung der Reaktionskinetik sind daher beide 


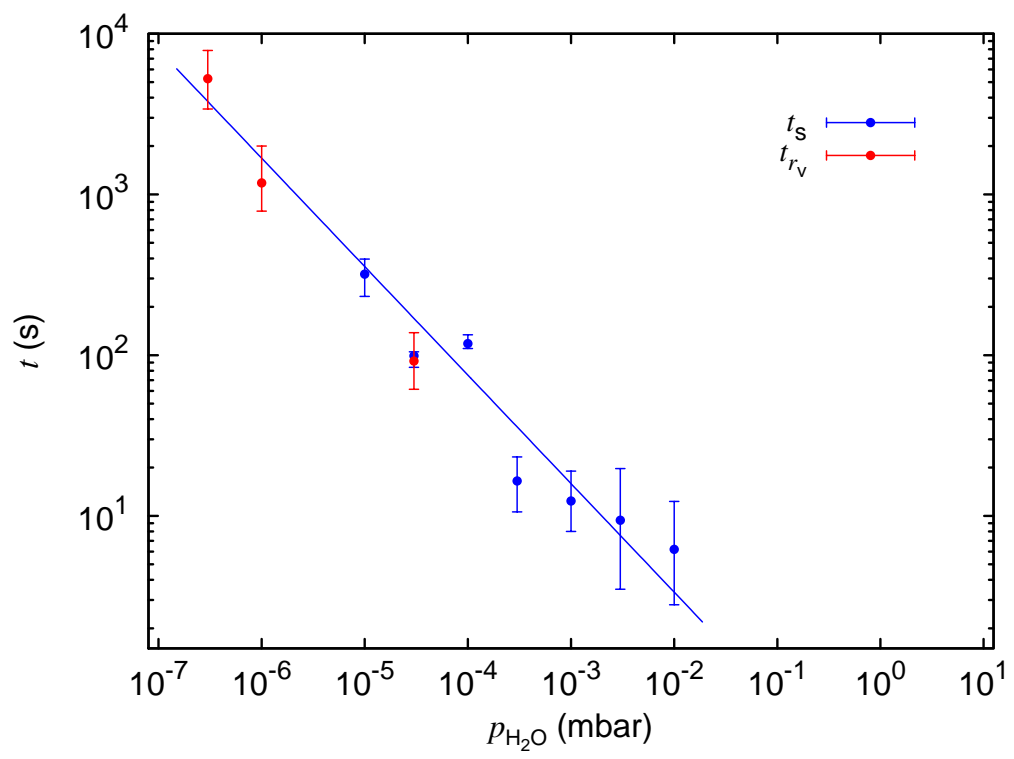

Abbildung 6.1: Auftragung zweier Zeiten, welche die Kinetik der Oxidation von W-Spitzen beschreiben, in Abhängigkeit von $\mathrm{H}_{2} \mathrm{O}$-Druck. Die Zeit $t_{\mathrm{s}}$, für die während der Oxidationsreaktion ein nahezu konstanter Strom messbar ist, ergibt sich aus Abb.4.7. Die Zeit $t_{r_{\mathrm{v}}}$, die vergeht bis $r_{\mathrm{v}}$ einen festen Wert annimmt, wurde aus Abb. 4.4 ermittelt. Dabei sind lediglich die Werte für Drücke unterhalb von $10^{-4}$ mbar berücksichtigt, da nur dort wie bei der Strommessung eine Spannung von $700 \mathrm{~V}$ an die Spitzen angelegt wurde. Der Fehler für $t_{\mathrm{s}}$ berücksichtigt nicht die unterschiedliche Spitzengeometrie.

Größen gleichermaßen geeignet.

\subsubsection{Kinetik des Anfangsstadiums}

Um zu klären, welcher Prozess die Kinetik zu Beginn der Oxidationsreaktion bestimmt, wird zunächst der Reaktionsstrom betrachtet. Nach 2.2.1 geht mit der Oxidationsreaktion stets ein Transport positiver Ladung von der Metall-Oxid-Grenzfläche zur Oxidoberfläche einher. Unabhängig davon, ob das Oxidwachstum durch Diffusion von Anionen oder Kationen durch das Oxid erfolgt, ist zur Bildung einer $\mathrm{WO}_{3}$-Einheit ein Transport von sechs Elementarladungen notwendig. Für jedes reagierende Sauerstoffatom müssen somit zwei Elementarladungen von der Oxidoberfläche abtransportiert werden - im vorliegenden Fall offenbar in den Gasraum, da ein Strom gemessen wird. Der maximal fließende Strom $I_{\mathrm{g}}$ kann somit durch die Auftreffrate von $\mathrm{H}_{2} \mathrm{O}$ auf die Spitze abgeschätzt werden, sofern die Fläche $F$ bekannt ist, auf der die Reaktion abläuft. Wird $\mathrm{H}_{2} \mathrm{O}$ als ideales Gas betrachtet, so folgt

$$
I_{\mathrm{g}}\left(T, p_{\mathrm{H}_{2} \mathrm{O}}, F\right)=\frac{p_{\mathrm{H}_{2} \mathrm{O}}}{\sqrt{2 \pi \mathrm{m}_{\mathrm{H}_{2} \mathrm{O}} \mathrm{kT}}} 2 \mathrm{e} F
$$

Eine Auftragung von $I_{\mathrm{g}}$ zusammen mit dem gemessenen Plateauwert des Stromes während der Oxidationsreaktion $I_{\mathrm{p}}$ in Abhängigkeit vom $\mathrm{H}_{2} \mathrm{O}$-Druck zeigt Abb. 6.2. Dabei wurde als Näherung für die Fläche $F$ zum einen die Projektion der Oxidoberfläche in Richtung der Symmetrieachse der Spitze $\left(F=\pi r_{\mathrm{v}}{ }^{2}\right)$ verwendet, zum anderen wurde unter der Annahme von Zylindersymmetrie die Oxidoberfläche mit der Finite-Elemente- 


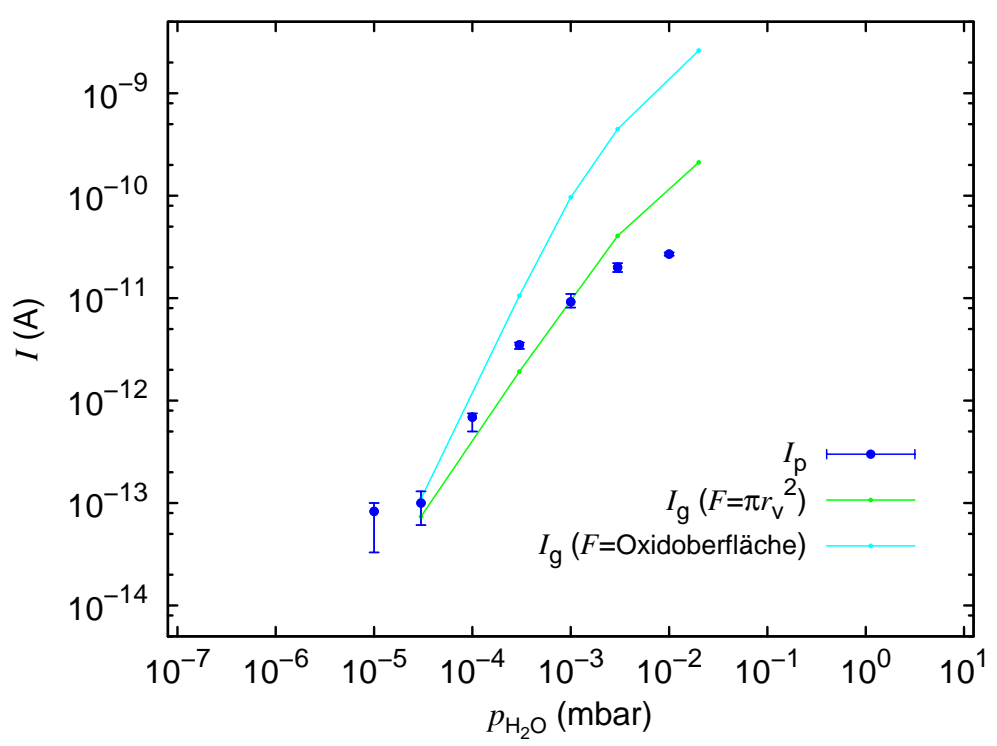

Abbildung 6.2: Auftragung des aus Abb. 4.7 bestimmten Plateauwert des Stromes während der Oxidationsreaktion $I_{\mathrm{p}}$ in Abhängigkeit von $\mathrm{H}_{2} \mathrm{O}$-Druck. Zusätzlich ist der gemäß (6.1) berechnete Strom $I_{\mathrm{g}}$ für die jeweils angegebenen Flächen $F$ dargestellt $(T=296 \mathrm{~K})$. Die Bestimmung von $F$ erfolgt durch Auswertung von TEM-Aufnahmen, welche nach Ablauf der Oxidationsreaktion angefertigt wurden. ${ }^{1}$

Methode bestimmt. Für Druckwerte von bis zu $10^{-3}$ mbar liegen $I_{\mathrm{g}}$ und $I_{\mathrm{p}}$ in der gleichen Größenordnung. Das Oxidwachstum ist demnach offenbar limitiert durch den Antransport von $\mathrm{H}_{2} \mathrm{O}$ und der Reaktionskoeffizient für $\mathrm{H}_{2} \mathrm{O}$ liegt nahe bei 1. Bei höheren Drücken hingegen wird offenbar ein anderer Schritt geschwindigkeitsbestimmend für die Oxidationsreaktion. Auch die qualitative Änderung der Spitzenmorphologie bei etwa $10^{-3} \mathrm{mbar}$ (siehe Abb. 4.6) weist auf einen solchen Übergang hin.

Sofern die Kinetik zu Beginn der Oxidationsreaktion bei niedrigen Drücken durch den Antransport von $\mathrm{H}_{2} \mathrm{O}$ bestimmt wird, sollte sich dies auch in der zeitlichen Entwicklung der Spitzenmorphologie widerspiegeln. Entscheidend für den Ablauf der Reaktion wäre dann die auf die Spitze einwirkende Dosis $\chi_{\mathrm{H}_{2} \mathrm{O}}=p_{\mathrm{H}_{2} \mathrm{O}} t$. Abb. 6.3 zeigt den Betrag der Längenänderung der metallischen W-Spitze auf der Symmetrieachse $\Delta z$ als Funktion der $\mathrm{H}_{2} \mathrm{O}$-Dosis. Nach einer anfänglich schnellen Zunahme von $\Delta z$ wird oberhalb einer gewissen Dosis ein nahezu stabiler Wert erreicht. Dieser Endwert nimmt dabei mit höherem $\mathrm{H}_{2} \mathrm{O}$-Druck zu. Obwohl der $\mathrm{H}_{2} \mathrm{O}$-Druck zwischen den einzelnen Proben um zwei Größenordnungen variiert, fallen die Werte für $\Delta z$ in der Phase des deutlichen Wachstums nahezu zusammen. Offenbar ist die $\mathrm{H}_{2} \mathrm{O}$-Dosis entscheidend für die Reaktionskinetik, und die Reaktion somit durch den Antransport von $\mathrm{H}_{2} \mathrm{O}$ begrenzt. Eine quantitative Abschätzung der Dosisabhängigkeit von $\Delta z$ lässt sich wieder aus der Auftreffrate von $\mathrm{H}_{2} \mathrm{O}$ auf der Spitze bestimmen. Bei Bildung von $\mathrm{WO}_{3}$ wird nach (2.2) durch jedes reagierende $\mathrm{H}_{2} \mathrm{O}-$ Molekül das Volumen der W-Spitze um $\frac{1}{6}$ des Volumens der Elementarzelle für Wolfram $\widetilde{\mathrm{V}}_{\mathrm{w}}$ verringert. Da im Druckbereich von $3 \cdot 10^{-7}$ mbar bis $3 \cdot 10^{-5}$ mbar $\mathrm{H}_{2} \mathrm{O}$

\footnotetext{
${ }^{1}$ Da die Fläche $F$ im Verlauf der Oxidationsreaktion zunimmt, liefert die Bestimmung von $F$ nach Ablauf der Reaktion zunächst einen zu großen Wert. In Abb. 4.7 fällt jedoch auf, dass trotz der Änderung von $F$ ein nahezu konstanter Strom gemessen wird. Zu Beginn der Oxidationsreaktion wird die geringere Fläche offenbar durch die erhöhte Auftreffrate von $\mathrm{H}_{2} \mathrm{O}$ auf der Spitze gemäß (6.3) kompensiert.
} 


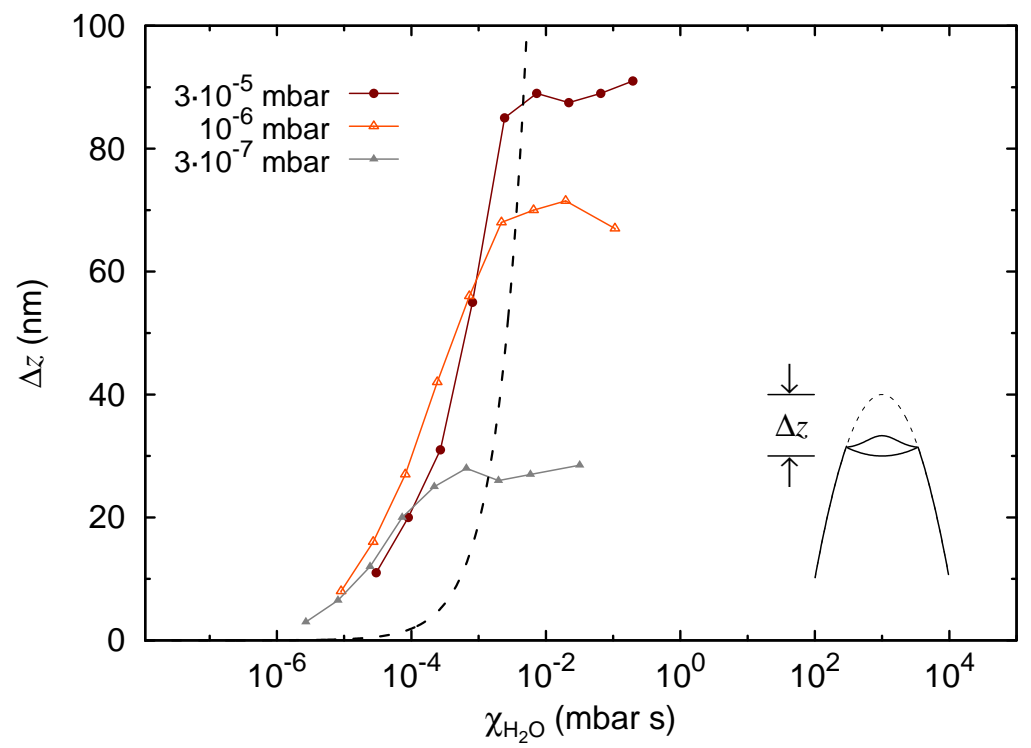

Abbildung 6.3: Aus dem Vergleich von TEM-Aufnahmen ermittelter Betrag der Längenänderung der metallischen Spitze auf der Symmetrieachse $\Delta z$ in Abhängigkeit von der $\mathrm{H}_{2} \mathrm{O}$-Dosis $\chi_{\mathrm{H}_{2} \mathrm{O}}$. Die unterbrochene Linie gibt den nach (6.2) erwarteten Verlauf wieder, jedoch ohne die durch das Feld erhöhte Auftreffrate gemäß (6.3). Der mit größer werdendem $\Delta z$ von $1 \mathrm{~nm}$ auf $4 \mathrm{~nm}$ zunehmende Fehler ist aus Gründen der Übersichtlichkeit nicht dargestellt.

lediglich ein geringe Menge Oxid auf der Spitze vorliegt, liegen sowohl die Oxidoberfläche als auch die Metall-Oxid-Grenzfläche nahezu senkrecht zur Symmetrieachse der Spitze. Der Betrag der Längenänderung der metallischen W-Spitze kann daher in guter Näherung in einem eindimensionalen Modell berechnet werden. Wird $\mathrm{H}_{2} \mathrm{O}$ wiederum als ideales Gas betrachtet, so folgt

$$
\Delta z\left(T, \chi_{\mathrm{H}_{2} \mathrm{O}}\right)=\frac{\widetilde{\mathrm{V}}_{\mathrm{W}}}{6 \sqrt{2 \pi \mathrm{m}_{\mathrm{H}_{2} \mathrm{O}} \mathrm{k} T}} \chi_{\mathrm{H}_{2} \mathrm{O}} .
$$

Die Auftragung des berechneten Verlaufs von $\Delta z$ als unterbrochene Linie in Abb. 6.3 zeigt, dass die Aufzehrung der W-Spitze in der Phase des deutlichen Wachstums erheblich schneller erfolgt als erwartet. Ursächlich für diese Abweichung ist vermutlich die erhöhte Auftreffrate des idealen Gases unter dem Einfluss des inhomogenen elektrischen Feldes in der Umgebung der Spitze. Aufgrund des permanenten Dipolmomentes des $\mathrm{H}_{2} \mathrm{O}$-Moleküles $\mathrm{d}_{\mathrm{H}_{2} \mathrm{O}}$ besteht eine attraktive Wechselwirkung zwischen der Spitze und den $\mathrm{H}_{2} \mathrm{O}-$ Molekülen. In Analogie zur der Berechnung von Eekelen [Eeke1970, S. 40] erhöht sich daher die Auftreffrate von $\mathrm{H}_{2} \mathrm{O}$ um einen Faktor

$$
\zeta\left(T, E_{\mathrm{o}}\right)=\frac{\mathrm{d}_{\mathrm{H}_{2} \mathrm{O}} E_{\mathrm{o}}}{\mathrm{k} T}+\exp \left(-\frac{\mathrm{d}_{\mathrm{H}_{2} \mathrm{O}} E_{\mathrm{o}}}{\mathrm{k} T}\right)
$$

wobei $E_{\mathrm{o}}$ den Betrag der Feldstärke an der Spitzenoberfläche bezeichnet. Die Erhöhung der Auftreffrate ist demnach im Wesentlichen durch das Verhältnis von Dipolenergie zu thermischer Energie bestimmt. Der zweite Summand sorgt lediglich für einen stetigen Übergang zu $\zeta=1 \mathrm{im}$ feldfreien Fall. Für eine Feldstärke $E_{\mathrm{o}}$ von etwa $4 \mathrm{~V} / \mathrm{nm}$, wie sie nach Abb. 4.16 bei Erreichen der stabilen Morphologie an der Spitzenoberfläche auftritt, 


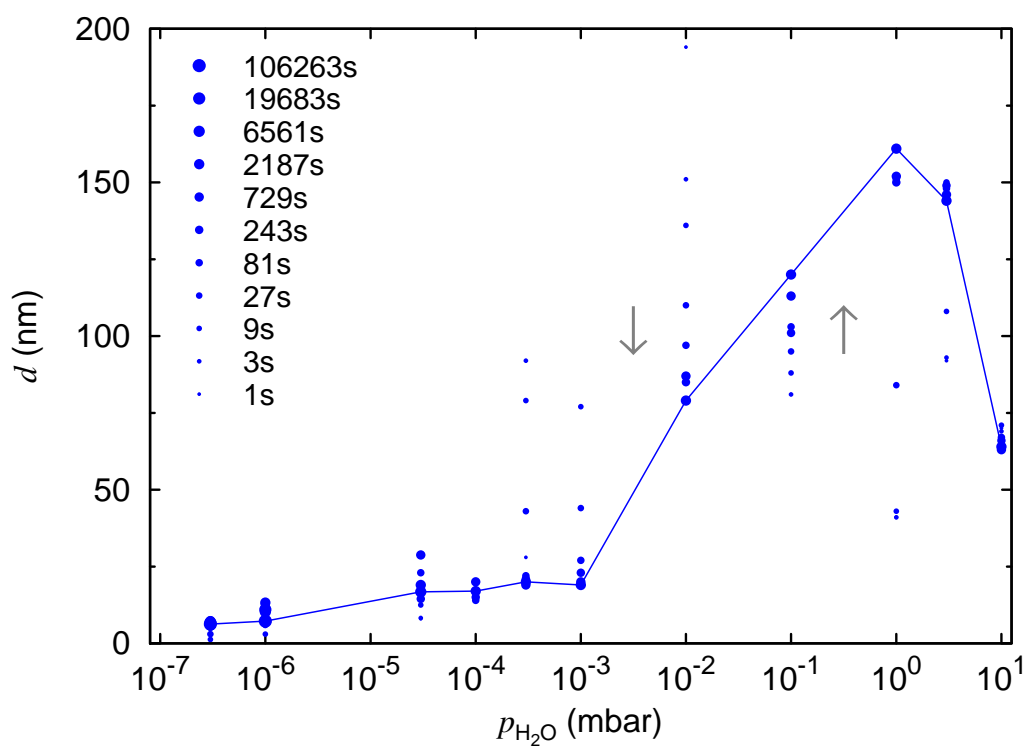

Abbildung 6.4: Dicke der Oxidschicht auf der Symmetrieachse $d$ in Abhängigkeit vom $\mathrm{H}_{2} \mathrm{O}-\mathrm{Druck}$ für verschiedene Reaktionszeiten. Die durchgehende Linie verbindet die Werte von $d$ für die jeweils maximale Reaktionszeit. Die Darstellung zeigt dieselben Daten wie Abb. 4.5.

folgt ein Faktor $\zeta=6 .^{2}$ Vor Erreichen der stabilen Morphologie ist bei geringerem $\Delta z$ jedoch auch $r_{\mathrm{v}}$ kleiner. Es liegt also eine höhere Feldstärke $E_{\mathrm{o}}$ an der Spitzenoberfläche vor. Daher nimmt, wie in Abb. 6.3 beobachtet, $\zeta$ mit geringerer $\mathrm{H}_{2} \mathrm{O}$-Dosis größere Werte an.

Sowohl der Stromfluss als auch die Morphologieentwicklung weisen somit darauf hin, dass für Drücke von höchstens $10^{-3}$ mbar $\mathrm{H}_{2} \mathrm{O}$ zunächst der Antransport von $\mathrm{H}_{2} \mathrm{O}$ der geschwindigkeitsbestimmende Schritt für die Oxidationsreaktion ist. Nachdem die Reaktion bis zu einem gewissen Grad abgelaufen ist, kommt sie jedoch zum Erliegen: Ein anderer Schritt wird geschwindigkeitsbestimmend. Bei hohem $\mathrm{H}_{2} \mathrm{O}$-Druck hingegen ist in Übereinstimmung mit der Messung von Oxidationsraten von bis zu $100 \mathrm{~nm} / \mathrm{s}$ an Silizium bei einem $\mathrm{H}_{2} \mathrm{O}$-Partialdruck im Bereich einiger mbar [Avou1997] die Kinetik der Reaktion auf der untersuchten Zeitskala nicht auflösbar.

\subsubsection{Morphologieentwicklung}

Für die Diskussion der Prozesse, welche zur beobachteten Morphologie von Spitze und Oxidschicht führen, sei zunächst nochmals auf Abb. $4.3 \mathrm{~b}$ ) verwiesen. Aus ihr wird deutlich, dass neben dem anfänglichen, durch den Antransport von Gas limitiertem Wachstum noch weitere Prozesse ablaufen, welche insbesondere einen Abtrag von Wolframoxid von der Spitze mit sich bringen. Zur übersichtlichen Darstellung dieses Effektes zeigt Abb. 6.4 die Dicke der Oxidschicht auf der Symmetrieachse $d$ in Abhängigkeit vom $\mathrm{H}_{2} \mathrm{O}-$ Druck. Bei einem $\mathrm{H}_{2} \mathrm{O}$-Druck von mindestens $10^{-1}$ mbar wird eine monotone Zunahme von $d$ mit der Zeit beobachtet. Für geringere Drücke hingegen folgt auf einen sehr schnel-

\footnotetext{
${ }^{2}$ Für das Dipolmoment von $\mathrm{H}_{2} \mathrm{O}$ im gasförmigen Zustand wurde nach [Shos1991] ein Wert von 6,19 Cm zugrunde gelegt.
} 


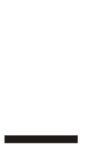

$50 \mathrm{~nm}$

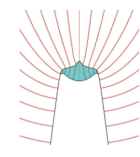

$10^{-6}$

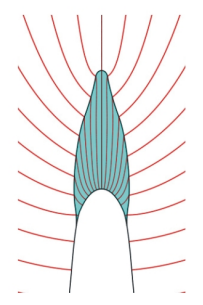

$10^{-2}$

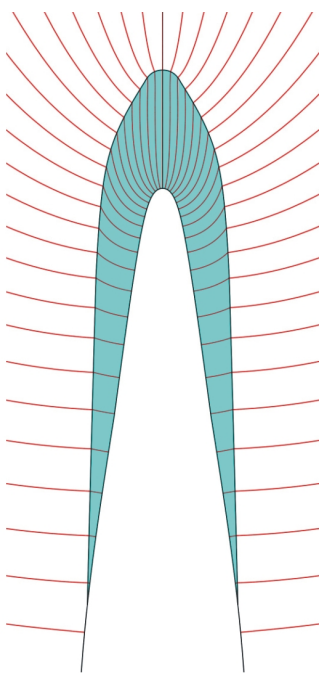

10 mbar

Abbildung 6.5: Mit der Finite-Elemente-Methode berechnete Feldlinien in der Umgebung verschiedener W-Spitzen, bei denen die Oxidationsreaktion bei dem jeweils angegebenen $\mathrm{H}_{2} \mathrm{O}-$ Druck für $2187 \mathrm{~s}$ erfolgte. Den Betrag der Feldstärke zeigt Abb. 4.16.

len Prozess, welcher zu großen $d$-Werten führt, ein zweiter Prozess, welcher $d$ wieder verringert.

Das Auftreten eines Wertes von $d=194 \mathrm{~nm}$ für eine Reaktionszeit von $1 \mathrm{~s}$ bei einem Druck von $10^{-2}$ mbar und $\Delta z=43 \mathrm{~nm}$ lässt sich nur durch Diffusion entlang der Oxidoberfläche verstehen. Als treibende Kraft für die Ausbildung scharfer Oxidspitzen wirkt hierbei das elektrische Feld. Sofern geladene Teilchen vorliegen, erfolgt ein Transport in Richtung abnehmenden elektrostatischen Potentials $\Phi$, bei neutralen Teilchen aufgrund der Wechselwirkung mit dem Dipolmoment in Richtung höherer Feldstärke $E$.

Der Verlust von Spitzenmaterial ist vermutlich auf Feldverdampfung zurückzuführen. Auch bei der massenspektrometrischen Untersuchung der Feldionisation von $\mathrm{H}_{2} \mathrm{O}$ an W-Spitzen [Schm1964] lassen sich Wolframoxid-Ionen nachweisen. Weiterhin deutet das Auftreten eines typischen, vom $\mathrm{H}_{2} \mathrm{O}$-Druck unabhängigen Krümmungsradius der Oxidspitzen von etwa $2 \mathrm{~nm}$ bei einer Feldstärke von etwa $6 \mathrm{~V} / \mathrm{nm}$ auf einen Feldverdampfungsprozess hin.

Innerhalb der Oxidschicht sollte die Diffusion von Anionen und Kationen im Wesentlichen entlang der Feldlinien erfolgen, da nach 6.3.2 das elektrische Feld die wesentliche Triebkraft für den Ablauf der Oxidationsreaktion bildet. Einen qualitativen Eindruck von den somit bevorzugten Diffusionspfaden gibt Abb.6.5. Wegen der starken Krümmung der Metall-Oxid-Grenzfläche ist dabei im Verlauf der Reaktion mit dem Aufbau mechanischer Spannungen zu rechnen, welche wiederum die Diffusion erheblich beeinflussen können [Avou1997, Ene2008, Liu1994, Sall2000].

Aus dem Zusammenwirken von Oberflächendiffusion, Feldverdampfung und Volumendiffusion ergibt sich folgende Modellvorstellung für die Entwicklung der Spitzenmorphologie: Bei niedrigem $\mathrm{H}_{2} \mathrm{O}$-Druck liegt die Reaktionsfront offenbar an der Oxidoberfläche. Da bei anodischer Oxidation von Wolfram die Transportzahlen von W-Kationen und O-Anionen in der gleichen Größenordnung liegen [Davi1965], ist bei dem um Größen- 
ordnungen geringerem $\mathrm{H}_{2} \mathrm{O}$-Partialdruck im Experiment eine deutliche Dominanz des Kationentransportes durch das Oxid zu erwarten. An der Oxidoberfläche erfolgt die Reaktion mit $\mathrm{H}_{2} \mathrm{O}$. Vor Ausbildung der maximalen Bindungszahl zu Sauerstoff $\left(\mathrm{WO}_{3}\right)$ erfolgt dabei Oberflächendiffusion unter dem Einfluss des elektrischen Feldes, da das Kation einen von Null verschiedenen Ladungszustand besitzt. ${ }^{3}$ Sofern ein Transport bis zum Apex der Oxidschicht stattfindet, kommt es dort zur Ausbildung einer feinen Oxidspitze bis schließlich die zum Einsatz der Feldverdampfung notwendige Feldstärke erreicht wird und Feldverdampfung erfolgt. ${ }^{4}$ Wird die maximale Bindungszahl zu Sauerstoff zuvor erreicht, verliert das Kation seine Nettoladung und die neu gebildete $\mathrm{WO}_{3}$-Einheit bleibt ortsfest. Da die Feldverdampfung thermisch aktiviert ist, kann dabei eine Verringerung von $d$ noch erfolgen, nachdem die eigentliche Oxidationsreaktion bereits zum Erliegen gekommen ist.

Bei hohem $\mathrm{H}_{2} \mathrm{O}$-Druck findet offenbar keine Oberflächendiffusion statt, die Ausbildung von scharfen Oxidspitzen wird nicht beobachtet. Dies kann zum einen auf eine derart hohe Auftreffrate von $\mathrm{H}_{2} \mathrm{O}$ auf der Spitze zurückzuführen sein, dass die mittlere freie Weglänge von Kationen an der Oxidoberfläche vernachlässigbar klein wird. Zum anderen kann sich die Reaktionsfront von der Oxidoberfläche in Richtung der Metall-OxidGrenzfläche verlagern, worauf Untersuchungen des Oxidationsverhaltens von Wolfram an Umgebungsatmosphäre [Ande1967] und die ähnlichen Transportzahlen von W-Kationen und O-Anionen bei anodischer Oxidation [Davi1965] hindeuten. In jedem Fall erfolgt der Transport von Sauerstoff und Wolfram im Wesentlichen entlang der Feldlinien, nicht jedoch entlang der Oxidoberfläche. Dadurch kommt es nicht zur Ausbildung scharfer Oxidspitzen und Feldverdampfung tritt nicht auf.

Nach der in Abb. 6.4 dargestellten zeitlichen Entwicklung von $d$ erfolgt der Übergang zwischen den beiden Reaktionsmechanismen im Bereich von $3 \cdot 10^{-2}$ mbar $\mathrm{H}_{2} \mathrm{O}$. Bei hinreichend geringem $\mathrm{H}_{2} \mathrm{O}$-Druck ist die mittlere freie Weglänge der Kationen schließlich so groß, dass sie stets den Apex der Oxidschicht erreichen können. Daher wird für Drücke unterhalb von $10^{-3}$ mbar keine Änderung der Spitzenmorphologie beobachtet. In Abb. 6.4 tritt für diesen Druck ein deutlicher Übergang in der für große Zeiten gemessenen Schichtdicke $d$ auf. Im Bereich hoher Drücke wird ein deutlicher Abfall der für große Zeiten gemessenen Schichtdicke beobachtet. Das Absinken von $d$ bei einem Druck von mehr als 1 mbar ist vermutlich auf eine Verlagerung der Reaktionsfront zur Metall-Oxid-Grenzfläche zurückzuführen.

Im Rahmen der Modellvorstellung für die Entwicklung der Spitzenmorphologie lässt sich auch die in 4.2.1 beschriebene Änderung der Krümmung der Metall-Oxid-Grenzfläche von konvex zu konkav verstehen: Mit abnehmendem Druck verlagert sich die Reaktionsfront zunehmend zur Oxidoberfläche. Der Übergang von Wolfram in das Oxid und der Transport von W-Kationen erfolgen daher bevorzugt an Orten hoher Feldstärke, wodurch sich Gebiete starker Krümmung der Metall-Oxid-Grenzfläche abbauen. Wenn die

\footnotetext{
${ }^{3}$ Nach Erreichen des maximalen Oxidationszustandes findet offenbar keine signifikante Oberflächendiffusion mehr statt, da sich die Morphologie der Oxidschichten bei hohen $\mathrm{H}_{2} \mathrm{O}$-Drücken auch für große Zeiten nicht messbar ändert. Insbesondere wird keine Ausbildung feiner Spitzen auf der Oxidschicht beobachtet.

${ }^{4}$ Ebenso kann Felddesorption von unterstöchimetrischem Wolframoxid erfolgen, da dies formal einen von Null verschiedenen Ladungszustand besitzt.
} 


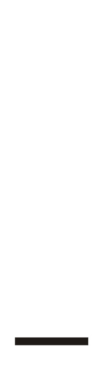

$50 \mathrm{~nm}$

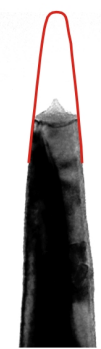

$10^{-4}$

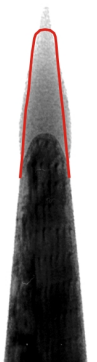

$10^{-2}$

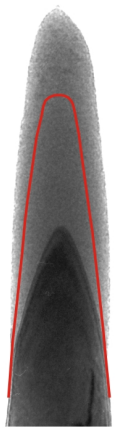

1 mbar

Abbildung 6.6: TEM-Aufnahmen dreier W-Spitzen, nachdem bei dem jeweils angegebenen $\mathrm{H}_{2} \mathrm{O}$-Druck für $729 \mathrm{~s}$ eine Spannung von $350 \mathrm{~V}$ an den Spitzen anlag. Die rote Linie gibt die Kontur der W-Spitzen vor Beginn der Oxidationsreaktion wieder.

Kinetik für Druckwerte von weniger als $10^{-3}$ mbar $\mathrm{H}_{2} \mathrm{O}$ durch den Antransport von $\mathrm{H}_{2} \mathrm{O}$ limitiert wird, stellt sie die Metall-Oxid-Grenzfläche schließlich so ein, dass an der Grenzfläche im Oxid eine nahezu konstante Feldstärke erreicht wird.

\subsubsection{Morphologie des stabilen Zustandes}

Bei der Diskussion der Morphologieentwicklung wurde bislang nicht betrachtet, inwieweit die Erhaltung der Teilchenzahl im Verlauf der Oxidationsreaktion gewährleistet ist. Ein Vergleich der Spitzenmorphologie nach Ablauf der Oxidationsreaktion mit der Morphologie vor Beginn der Reaktion in Abb. 6.6 zeigt, dass bei geringem $\mathrm{H}_{2} \mathrm{O}$-Druck in erheblichem Umfang Spitzenmaterial verloren geht. Zur Quantifizierung dieses Effektes wurde durch numerische Integration das Volumen des Oxides auf der Spitze $\widetilde{V}_{\mathrm{O}}$ sowie das Volumen des im Verlauf der Oxidationsreaktion aufgezehrten Metalls $\widetilde{V}_{\mathrm{M}}$ auf Grundlage der jeweiligen TEM-Aufnahmen bestimmt. Das Verhältnis von Oxidvolumen zum Volumen des aufgezehrten Metalls $R:=\widetilde{V}_{\mathrm{O}} / \widetilde{V}_{\mathrm{M}}$ ist in Abb. 6.7 dargestellt. Mit abnehmendem $\mathrm{H}_{2} \mathrm{O}$-Druck ist eine erhebliche Verringerung von $R$ zu beobachten, wobei schließlich Werte deutlich kleiner als 1 auftreten. Somit weist auch die Bilanz der Teilchenzahl auf einen Verlust von Spitzenmaterial hin und liefert einen Beleg dafür, dass Feldverdampfung tatsächlich stattfindet.

Der bei der Bildung von $\mathrm{M}_{\mathrm{x}} \mathrm{O}_{\mathrm{y}}$ zu erwartende Wert von $R$ ergibt sich nach [Pill1923] $\mathrm{zu} \mathrm{R}_{\mathrm{M}_{\mathrm{x}} \mathrm{O}_{\mathrm{y}}}=\left(\rho_{\mathrm{M}} \widetilde{\mathrm{M}}_{\mathrm{M}_{\mathrm{x}} \mathrm{O}_{\mathrm{y}}}\right) /\left(\mathrm{x} \rho_{\mathrm{M}_{\mathrm{x}} \mathrm{O}_{\mathrm{y}}} \widetilde{\mathrm{M}}_{\mathrm{M}}\right)$, wobei $\rho_{\mathrm{i}}$ die Dichte der Substanz i und $\widetilde{\mathrm{M}}_{\mathrm{i}}$ die Molmasse der Spezies i bezeichnet. Für die thermodynamisch stabilen Wolframoxide folgt $\mathrm{R}_{\mathrm{WO}_{2}}=2,09$ und $\mathrm{R}_{\mathrm{WO}_{3}}=3,34$. Der Vergleich mit den experimentell bestimmten Werten für $R$ in Abb. 6.7 zeigt, dass für einen $\mathrm{H}_{2} \mathrm{O}$-Druck oberhalb von $3 \cdot 10^{-2}$ mbar, also in einem Druckbereich, in dem nach 6.1.2 keine Feldverdampfung stattfindet, $\mathrm{WO}_{3}$ gebildet wird. Dies steht in Übereinstimmung mit der Bestimmung der Stöchiometrie des Wolframoxides mittels Laser-unterstützte Atomsondentomographie in 4.3.1. Der Abfall von $R$ bei geringeren Drücken ist dann offenbar nicht auf eine Änderung der Oxidstöchiometrie, sondern auf Materialverlust durch Feldverdampfung zurückzuführen. 


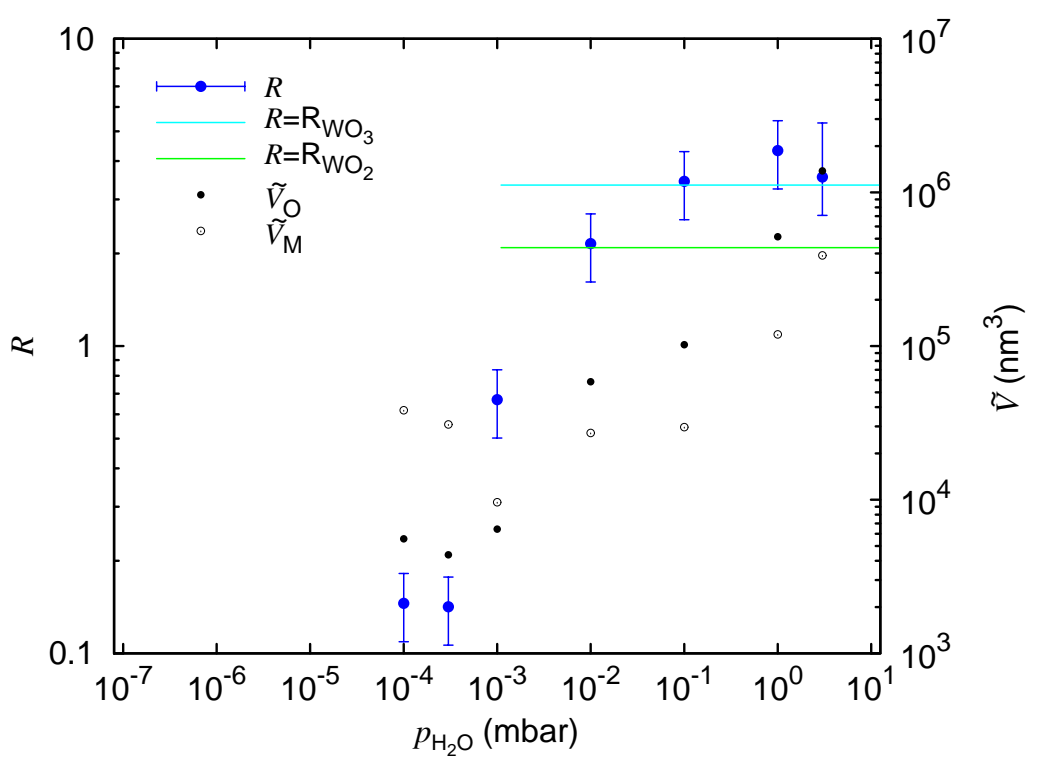

Abbildung 6.7: Doppelt-logarithmische Darstellung des Verhältnisses von Oxidvolumen zum Volumen des aufgezehrten Metalls $R$ in Abhängigkeit vom $\mathrm{H}_{2}$ O-Druck. An den W-Spitzen lag jeweils für 729s eine Spannung von $350 \mathrm{~V}$ an. Zusätzlich sind die für $\mathrm{WO}_{2}$ und $\mathrm{WO}_{3}$ berechneten Werte von $R$ angegeben. Die durch numerische Integration bestimmten Volumina $\widetilde{V}_{\mathrm{O}}$ und $\widetilde{V}_{\mathrm{M}}$ sind der rechten Ordinatenachse zu entnehmen.

\subsection{Aggregatzustand des $\mathrm{H}_{2} \mathrm{O}$ auf der Spitze}

Durch die Wechselwirkung zwischen dem elektrischen Feld in der Umgebung der Spitze und dem permanenten Dipolmoment des $\mathrm{H}_{2} \mathrm{O}$ erhöht sich, wie bereits in 6.1 .1 berücksichtigt, die Auftreffrate von $\mathrm{H}_{2} \mathrm{O}$ auf der Spitze. Somit ist der effektive Partialdruck in der unmittelbaren Spitzenumgebung erhöht. Um zu klären, ob der Anstieg des Partialdruckes von $\mathrm{H}_{2} \mathrm{O}$ derart stark ist, dass bereits Kondensation auf der Spitze erfolgt, also eine elektrochemische Reaktion zwischen dem Spitzenmaterial und $\mathrm{H}_{2} \mathrm{O}(\mathrm{l})$ abläuft, werden im Folgenden Ratengleichungen für die einzelnen Reaktionsschritte des $\mathrm{H}_{2} \mathrm{O}$ betrachtet.

Die Auftreffrate von $\mathrm{H}_{2} \mathrm{O}$ auf der Spitze ist nach (6.3) um den Faktor $\zeta$ erhöht. Da nach 6.1.1 der Reaktionskoeffizient für die Oxidationsreaktion nahe bei 1 liegt, ist auch ein Haftungskoeffizient für $\mathrm{H}_{2} \mathrm{O}$ von etwa 1 anzunehmen. Die Adsorptionsrate von $\mathrm{H}_{2} \mathrm{O}$ sollte demnach der Auftreffrate entsprechen. Für die auf der Spitze adsorbierte Teilchenstromdichte $j_{\mathrm{H}_{2} \mathrm{O}}^{\text {ad }}$ folgt somit

$$
j_{\mathrm{H}_{2} \mathrm{O}}^{\mathrm{ad}}\left(T, E_{\mathrm{o}}, p_{\mathrm{H}_{2} \mathrm{O}}\right)=\left(\frac{\mathrm{d}_{\mathrm{H}_{2} \mathrm{O}} E_{\mathrm{o}}}{\mathrm{k} T}+\exp \left(-\frac{\mathrm{d}_{\mathrm{H}_{2} \mathrm{O}} E_{\mathrm{o}}}{\mathrm{k} T}\right)\right) \frac{p_{\mathrm{H}_{2} \mathrm{O}}}{\sqrt{2 \pi \mathrm{m}_{\mathrm{H}_{2} \mathrm{O}} \mathrm{k} T}} .
$$

Die thermisch aktivierte Desorption von $\mathrm{H}_{2} \mathrm{O}$ erfolgt unter dem Einfluss eines elektrischen Feldes mit geringerer Rate, da sich die Energiebarriere um den Betrag $\mathrm{d}_{\mathrm{H}_{2} \mathrm{O}} E_{\mathrm{o}}$ erhöht. Im feldfreien Fall liegt die Verdampfungsenthalpie von $\mathrm{H}_{2} \mathrm{O}(\mathrm{l})$ am Tripelpunkt bei $0,47 \mathrm{eV}$; die bei der Adsorption von $\mathrm{H}_{2} \mathrm{O}$ auf Oxidoberflächen freiwerdende Energie beträgt nach [Thie1987] $0,4 \mathrm{eV}$ bis $0,6 \mathrm{eV}$. Unabhängig davon, ob Kondensation von $\mathrm{H}_{2} \mathrm{O}$ auf der Spitze erfolgt oder lediglich Adsorption von $\mathrm{H}_{2} \mathrm{O}$ auf der oxidischen Spitze 
stattfindet, kann somit ein Wert von $0,47 \mathrm{eV}$ für die Energiebarriere bei Desorption im feldfreien Fall $\mathrm{Q}^{\text {de }}$ angesetzt werden. Bei einer Flächendichte von adsorbiertem $\mathrm{H}_{2} \mathrm{O}$ von $\theta_{\mathrm{H}_{2} \mathrm{O}}$ folgt somit für die Desorption eine Teilchenstromdichte von

$$
j_{\mathrm{H}_{2} \mathrm{O}}^{\mathrm{de}}\left(T, E_{\mathrm{O}}, \theta_{\mathrm{H}_{2} \mathrm{O}}\right)=\nu \exp \left(-\frac{\mathrm{Q}^{\mathrm{de}}+\mathrm{d}_{\mathrm{H}_{2} \mathrm{O}} E_{\mathrm{O}}}{\mathrm{k} T}\right) \theta_{\mathrm{H}_{2} \mathrm{O}} .
$$

Feldionisation und Felddesorption von Ionen finden unter den vorliegenden experimentellen Bedingungen offenbar nicht statt, da nach Ablauf der Oxidationsreaktion kein Strom messbar ist. Auch die gute Übereinstimmung von gemessenem und berechnetem $\Delta z$ in Abb. 6.3 deutet darauf hin, dass Feldionisation und Felddesorption nicht in signifikantem Maße ablaufen. Andernfalls wäre eine deutliche Verringerung der für die Oxidationsreaktion zur Verfügung stehenden Konzentration an $\mathrm{H}_{2} \mathrm{O}$ zu erwarten, der gemessene Wert von $\Delta z$ sollte entsprechend geringer als der berechnete sein. Auch Schmidt [Schm1964] beobachtet bei der massenspektrometrischen Untersuchung der Feldionisation von $\mathrm{H}_{2} \mathrm{O}$ an W-Spitzen das Auftreten von $\mathrm{H}_{2} \mathrm{O}^{+}$und hydratisiertem $\mathrm{H}^{+}$erst bei Feldstärken oberhalb von $6 \mathrm{~V} / \mathrm{nm}^{5}$ Ein auf Feldionisation und Felddesorption zurückzuführender Teilchenstrom wird daher nicht betrachtet.

Der durch die eigentliche Oxidationsreaktion verursachte Teilchenstrom von $\mathrm{H}_{2} \mathrm{O}$ wird im Allgemeinen von der Feldstärkeverteilung im Oxid sowie der lokalen Konzentration der diffundierenden und reagierenden Spezies abhängen. Ein Ausdruck für die Flächendichte von $\mathrm{H}_{2} \mathrm{O}$ an der Oxidoberfläche lässt sich daher nur für den Fall angeben, dass die Oxidationsreaktion zum Erliegen gekommen ist und sich an der Oxidoberfläche ein dynamischer Gleichgewichtszustand eingestellt hat. Dann gilt $\frac{\mathrm{d}}{\mathrm{dt}} \theta_{\mathrm{H}_{2} \mathrm{O}}=j_{\mathrm{H}_{2} \mathrm{O}}^{\mathrm{ad}}-j_{\mathrm{H}_{2} \mathrm{O}}^{\mathrm{de}}=0$. Mit (6.4) und (6.5) folgt für den $\mathrm{H}_{2} \mathrm{O}$-Druck, der notwendig ist, damit bei gegebener Feldstärke an der Oxidoberfläche $E_{\mathrm{o}}$ eine Flächendichte $\theta_{\mathrm{H}_{2} \mathrm{O}}$ an der Oxidoberfläche erreicht wird

$$
p_{\mathrm{H}_{2} \mathrm{O}}=\nu \theta_{\mathrm{H}_{2} \mathrm{O}} \sqrt{2 \pi \mathrm{m}_{\mathrm{H}_{2} \mathrm{O}} \mathrm{k} T} \exp \left(-\frac{\mathrm{Q}^{\mathrm{de}}}{\mathrm{k} T}\right)\left(\frac{\mathrm{d}_{\mathrm{H}_{2} \mathrm{O}} E_{\mathrm{o}}}{\mathrm{k} T} \exp \left(\frac{\mathrm{d}_{\mathrm{H}_{2} \mathrm{O}} E_{\mathrm{o}}}{\mathrm{k} T}\right)+1\right)^{-1} .
$$

Eine Auftragung von $p_{\mathrm{H}_{2} \mathrm{O}}$ in Abhängigkeit von $E_{\mathrm{o}}$ in Abb. 6.8 macht deutlich, dass mit steigender Feldstärke $E_{\mathrm{O}}$ der zum Erreichen einer gegebenen Flächendichte $\theta_{\mathrm{H}_{2} \mathrm{O}}$ notwendige Druck erheblich abfällt. Wird eine Flächendichte von einer Monolage (ML) $\mathrm{H}_{2} \mathrm{O}$ im feldfreien Fall bei 28 mbar erreicht, so ist bei einer Feldstärke von $5 \mathrm{~V} / \mathrm{nm}$ dazu nur noch ein Druck von $2 \cdot 10^{-3}$ mbar notwendig. Für geringere Flächendichten folgen entsprechend niedrigere Druckwerte.

Zur Klärung der Frage, ob die Oxidationsreaktion als Reaktion zwischen einem Festkörper und $\mathrm{H}_{2} \mathrm{O}(\mathrm{l})$ oder aber als Reaktion zwischen einem Festkörper und an der Oxidoberfläche adsorbiertem $\mathrm{H}_{2} \mathrm{O}$ zu betrachten ist, sind in Abb. 6.8 zusätzlich die Werte $E_{r_{\mathrm{v}}}^{\mathrm{g}}\left(p_{\mathrm{H}_{2} \mathrm{O}}\right)$ aus Abb. 4.17 eingetragen. ${ }^{6}$ Für Druckwerte oberhalb von $10^{-1}$ mbar liegt die

\footnotetext{
${ }^{5}$ Die Angaben für die Einsatzfeldstärke von Schmidt [Schm1964] basieren auf der Ausgangsmorphologie der Spitzen und geben daher für Wolfram die Verhältnisse nach Beginn der Reaktion nicht angemessen wieder.

${ }^{6}$ Die Beschränkung auf die Feldstärke an der Oxidoberfläche an der Position $r_{\mathrm{v}}$ geschieht hier aus Gründen der Übersichtlichkeit. Auch bei Berücksichtigung der Feldstärkeverteilung entlang der Oxidoberfläche folgt qualitativ das gleiche Verhalten.
} 


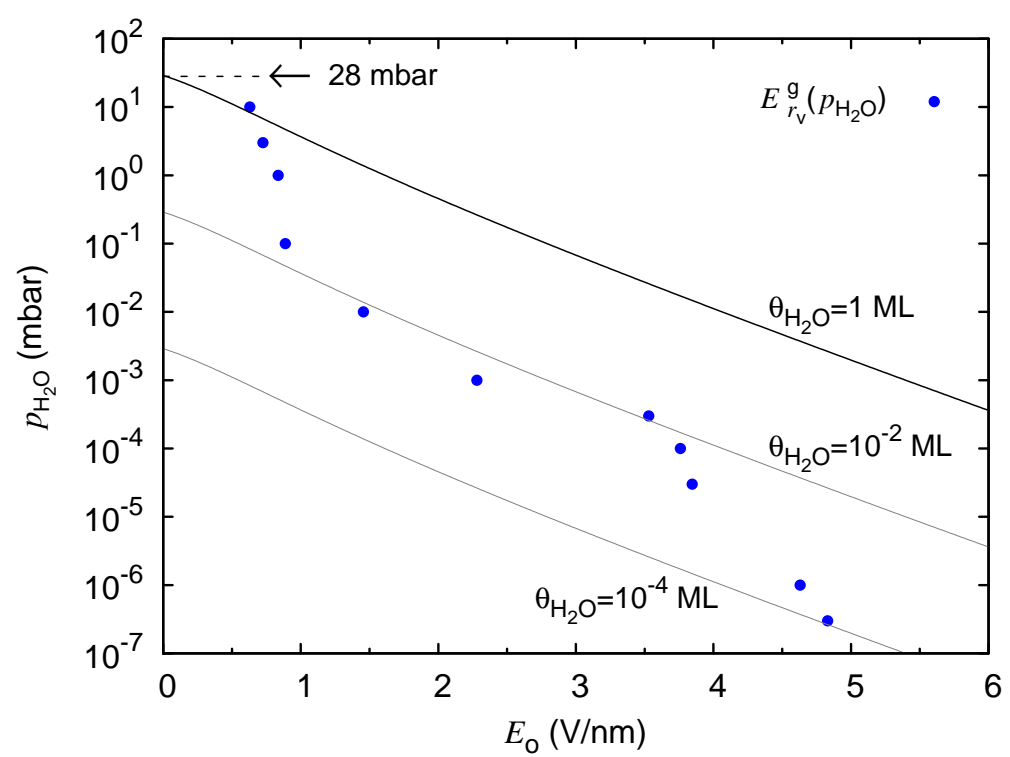

Abbildung 6.8: Auftragung des $\mathrm{H}_{2} \mathrm{O}$-Druckes, der notwendig ist, damit bei gegebener Feldstärke an der Oxidoberfläche $E_{\mathrm{O}}$ gemäß (6.6) eine Flächendichte $\theta_{\mathrm{H}_{2} \mathrm{O}}$ an der Oxidoberfläche erreicht wird. Der Wert $\nu=2 \cdot 10^{15} \mathrm{~s}^{-1}$ wurde so gewählt, dass bei einer Temperatur von $T=296 \mathrm{~K}$ und $\mathrm{Q}^{\text {de }}=0,47 \mathrm{eV}$ für eine Flächendichte von $1 \mathrm{ML}\left(5 \cdot 10^{18} \mathrm{~m}^{-2}\right)$ im feldfreien Fall gerade der Gleichgewichtspartialdruck von 28 mbar $\mathrm{H}_{2} \mathrm{O}$ resultiert. Zusätzlich ist die mit der Finite-Elemente-Methode bestimmte Feldstärke an der Oxidoberfläche im Gasraum am Ort verschwindender Oxiddicke $E_{r_{\mathrm{v}}}^{\mathrm{g}}$ aus Abb.4.17 eingetragen.

Flächendichte von $\mathrm{H}_{2} \mathrm{O}$ an der Position $r_{\mathrm{v}}$ in der Größenordnung von $1 \mathrm{ML}$ und damit im Grenzbereich zur Kondensation von $\mathrm{H}_{2} \mathrm{O}$. Für geringere Druckwerte beträgt die Flächendichte von $\mathrm{H}_{2} \mathrm{O}$ lediglich $10^{-4} \mathrm{ML}$ bis $10^{-2}$ ML. Nachdem die Oxidationsreaktion zum Erliegen gekommen ist, liegt an der Position $r_{\mathrm{v}}$ somit offenbar kein $\mathrm{H}_{2} \mathrm{O}(\mathrm{l})$ vor. Auch im Verlauf der Oxidationsreaktion ist im Allgemeinen keine Kondensation von $\mathrm{H}_{2} \mathrm{O}$ auf der Spitze zu erwarten: Der Wert von $E_{r_{\mathrm{v}}}^{\mathrm{g}}$ nimmt mit größer werdendem $r_{\mathrm{v}}$ stetig ab und liegt folglich lang bevor die Reaktion zum Erliegen kommt unter dem für Kondensation von $\mathrm{H}_{2} \mathrm{O}$ notwendigen Wert.

Für Druckwerte von maximal $10^{-1}$ mbar $\mathrm{H}_{2} \mathrm{O}$ ist die Oxidation von W-Spitzen somit als eine Reaktion zwischen einem Festkörper und adsorbiertem $\mathrm{H}_{2} \mathrm{O}$ zu betrachten. Für Al-Spitzen folgt aus einem Vergleich von Abb. 6.8 und den Daten in Abb. 5.7 ebenfalls, dass bei geringem Druck keine Kondensation von $\mathrm{H}_{2} \mathrm{O}$ auf der Spitze erfolgt. Liegt der $\mathrm{H}_{2} \mathrm{O}$-Druck hingegen oberhalb von $10^{-1} \mathrm{mbar}$, so ist bei Al-Spitzen Kondensation von $\mathrm{H}_{2} \mathrm{O}$ zu erwarten. Die Modellvorstellung zum Aggregatzustand des $\mathrm{H}_{2} \mathrm{O}$ auf der Spitze steht somit in Übereinstimmung mit den in 2.2.4 vorgestellten AFM-Studien zur Tieftemperaturoxidation, welche bei hohem $\mathrm{H}_{2} \mathrm{O}$-Partialdruck ebenfalls auf die Existenz eines Wassermeniskus zwischen Spitze und Probe hinweisen.

\subsection{Einfluss des Feldes auf die Oxidationsreaktion}

In diesem Abschnitt wird näher betrachtet in welcher Weise das externe elektrische Feld die Oxidationsreaktion beeinflusst. Zunächst wird die in 4.4.1 beschriebene Existenz ei- 


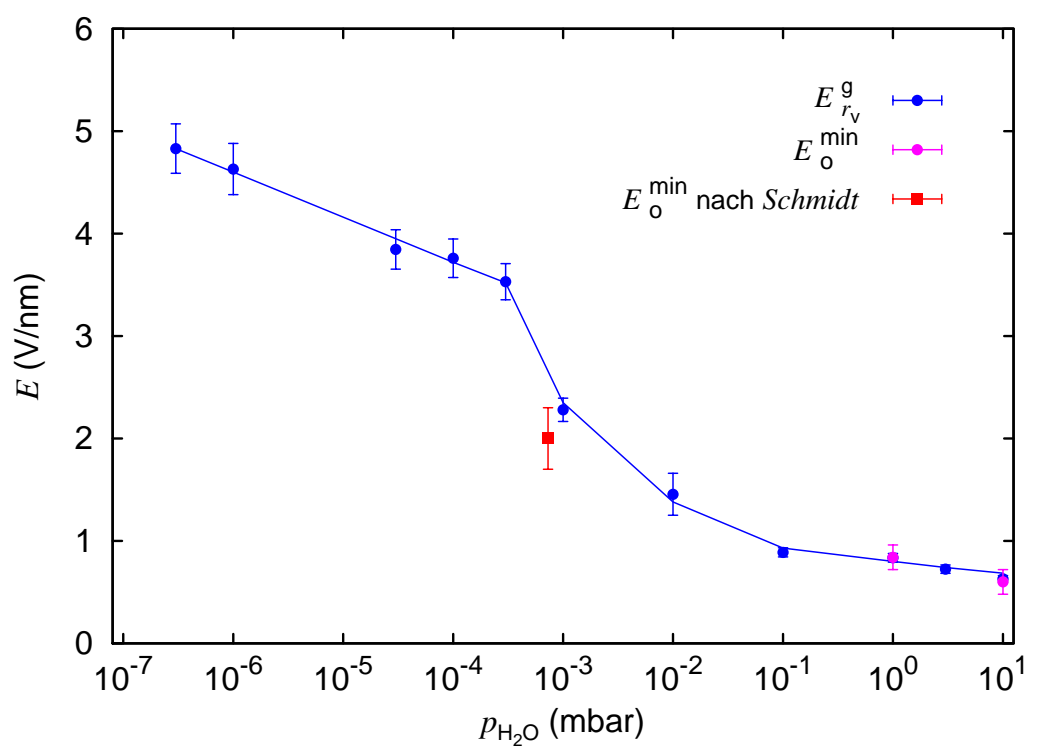

Abbildung 6.9: Darstellung der für die Oxidationsreaktion von W-Spitzen charakteristischen Feldstärken in Abhängigkeit vom $\mathrm{H}_{2} \mathrm{O}$-Druck. Die Feldstärke an der Oxidoberfläche am Ort verschwindender Oxiddicke $E_{r_{\mathrm{v}}}^{\mathrm{g}}$ ist aus Abb 4.17 übernommen. Die Werte von $E_{\mathrm{o}}^{\min }$ folgen aus Abb. 4.15. Sie wurden durch Skalierung der Einsatzfeldstärke für He-Ionisation von $24 \mathrm{~V} / \mathrm{nm}$ [Mill1996, S. 58] mit dem Verhältnis zwischen der Einsatzspannung für die Oxidationsreaktion und der Spannung bei der Präparation der Proben im FIM $(5000 \mathrm{~V})$ ermittelt. ${ }^{7}$ Zusätzlich ist die Grenzfeldstärke angegeben von der an Schmidt [Schm1964] das Auftreten eines Ionenstromes bei der massenspektrometrischen Untersuchung der Feldionisation von $\mathrm{H}_{2} \mathrm{O}$ an W-Spitzen beobachtet.

ner für den Ablauf der Oxidationsreaktion notwendigen Mindestfeldstärke eingehender diskutiert. Im Anschluß wird die Thermodynamik der Oxidationsreaktion unter dem Einfluss eines elektrischen Feldes betrachtet. Da die feldinduzierte Oxidationsreaktion thermodynamisch stets begünstigt ist, muss die Oxidationsreaktion von einem gewissen Stadium an kinetisch gehemmt sein. Daher wird abschließend der Feldeinfluss auf die einzelnen Teilschritte der Oxidationsreaktion diskutiert, wodurch der geschwindigkeitsbestimmende Schritt der Oxidationsreaktion identifiziert werden kann.

\subsubsection{Existenz einer kritischen Feldstärke für die Reaktion}

Nach den Ergebnissen aus Kapitel 4 existieren zwei kritische Feldstärken, welche charakteristisch für die Oxidationsreaktion von W-Spitzen sind: Zum einen ist aus 4.1 bekannt, dass für den Ablauf der Oxidationsreaktion eine vom $\mathrm{H}_{2} \mathrm{O}$-Druck abhängige Mindestfeldstärke $E_{\mathrm{o}}^{\min }$ notwendig ist. Zum anderen wird beobachtet, dass die Oxidationsreaktion zum Erliegen kommt, nachdem sie bis zu einem gewissen Grad abgelaufen ist. Die sich so einstellende Spitzenmorphologie ist nach 4.4.1 durch das Auftreten einer konstanten Feldstärke am Ort verschwindender Oxiddicke $E_{r_{\mathrm{v}}}^{\mathrm{g}}$ gekennzeichnet. Auch $E_{r_{\mathrm{v}}}^{\mathrm{g}}$ weist dabei eine deutliche Abhängigkeit vom $\mathrm{H}_{2} \mathrm{O}$-Druck auf.

Abb. 6.9 zeigt einen Vergleich der Werte von $E_{\mathrm{o}}^{\min }$ und $E_{r_{\mathrm{v}}}^{\mathrm{g}}$ in Abhängigkeit vom $\mathrm{H}_{2} \mathrm{O}-$ Druck. Bei hohem Druck ist die Einsatzfeldstärke für die Oxidationsreaktion identisch

\footnotetext{
${ }^{7}$ Es wird die Einsatzfeldstärke für He-Ionisation zugrunde gelegt, da die W-Spitzen im FIM nicht
} 
mit derjenigen Feldstärke, bei der die Reaktion zum Erliegen kommt. Für einen Druck von $7 \cdot 10^{-4}$ mbar $\mathrm{H}_{2} \mathrm{O}$ zeigt Abb. 6.9 die von Schmidt [Schm1964] angegebene Einsatzfeldstärke für Feldionisation von $\mathrm{H}_{2} \mathrm{O}$ an W-Spitzen. Wegen des Auftretens von Oxidionen in den von Schmidt gemessenen Massenspektren ist diese Feldstärke offenbar identisch mit der Mindestfeldstärke, welche für die Oxidationsreaktion notwendig ist. Das Auftreten von Oxidionen steht dabei in Übereinstimmung mit der in 6.1.3 gemachten Beobachtung, dass für einen $\mathrm{H}_{2} \mathrm{O}$-Druck von weniger als $3 \cdot 10^{-2}$ mbar Feldverdampfung des Oxides erfolgt. Auch bei einem $\mathrm{H}_{2} \mathrm{O}$-Druck von $10^{-3}$ mbar sind $E_{\mathrm{o}}^{\mathrm{min}}$ und $E_{r_{\mathrm{v}}}^{\mathrm{g}}$ somit offenbar identisch. Die Abhängigkeit der Oxidationsreaktion vom äußeren elektrischen Feld kann folglich gleichermaßen durch $E_{\mathrm{o}}^{\mathrm{min}}$ und durch $E_{r_{\mathrm{v}}}^{\mathrm{g}}$ beschrieben werden.

Für die Oxidationsreaktion existiert in beiden Fällen keine Keimbildungsbarriere, da auf der Spitzenoberfläche stets das natürliche Oxid vorhanden ist. An der Position $r_{\mathrm{v}}$ liegt darüber hinaus noch das unter dem Einfluss des externen Feldes gebildete Oxid vor. Da zudem $E_{r_{\mathrm{v}}}^{\mathrm{g}}$ experimentell mit höherer Genauigkeit bestimmt werden kann, wird im Folgenden dieser Wert betrachtet. Weiterhin ist die Position $r_{\mathrm{v}}$ dadurch ausgezeichnet, dass aufgrund der beliebig geringen Oxidschichtdicke die Tangentialkomponente der Feldstärke vernachlässigbar ist. Lokal kann daher die Oxidationsreaktion an der Position $r_{\mathrm{v}}$ als eindimensionales Problem betrachtet werden.

\subsubsection{Thermodynamik der feldinduzierten Oxidationsreaktion}

Das externe elektrische Feld beeinflusst die Thermodynamik der Oxidationsreaktion in zwei Formen: Zum einen tritt der Term ze $\Phi$ zum chemischen Potential der einzelnen Spezies hinzu, zum anderen kann sich die Aktivität der einzelnen Spezies erheblich ändern. Insbesondere die Konzentration des $\mathrm{H}_{2} \mathrm{O}$ an der Oxidoberfläche weicht nach der Betrachtung in 6.2 erheblich von derjenigen im feldfreien Fall ab.

Die Reaktionsgleichung für die Bildung von $\mathrm{WO}_{3}$ ergibt sich nach $2.2 \mathrm{zu}$

$$
\frac{1}{3} \mathrm{~W}(\mathrm{~s})+\mathrm{H}_{2} \mathrm{O}(\mathrm{g}) \rightarrow \frac{1}{3} \mathrm{WO}_{3}(\mathrm{~s})+2 \mathrm{e}^{-}+2 \mathrm{H}^{+} .8
$$

Die Aufspaltung in Elektronen und H-Ionen wurde dabei vorgenommen, da beide Spezies nach 2.2.1 an unterschiedlichen Orten entstehen: Die Elektronen werden beim Übergang des Wolframs in das Oxid frei, die H-Ionen entstehen bei der Reaktion des $\mathrm{H}_{2} \mathrm{O}$ an der Oxidoberfläche. Einen experimentellen Beleg für die Trennung der Ladungen liefert die Strommessung während der Oxidationsreaktion. Die gemessene Ladung entspricht derjenigen Ladungsmenge die sich ergibt, wenn aus dem Volumen des aufgezehrten Wolfram die Zahl der W-Atome bestimmt wird und für jedes Atom die Desorption von sechs Elementarladungen von der Oxidoberfläche angesetzt wird.

Für die Thermodynamik der Reaktion (6.7) ergibt sich nun Folgendes: Da die Elektronen bereits am Ort des maximalen Potentials $\Phi=V$ entstehen, erhöht sich die Triebkraft für den Elektronenübergang durch das elektrische Feld nicht. Die H-Ionen hingegen durchlaufen auf ihrem Weg von der Oxidoberfläche zur Gegenelektrode eine

ausentwickelt wurden, sondern lediglich ein Teil der Spitzenoberfläche im FIM sichtbar war.

${ }^{8}$ Die Reaktionsgleichung (6.7) ist als Netto-Gleichung zu verstehen. Insbesondere ist statt der Desorption von $\mathrm{H}^{+}$auch Desorption von $\mathrm{H}_{3} \mathrm{O}^{+}$oder stärker hydratisiertem $\mathrm{H}^{+}$möglich. 
Potentialdifferenz in der Größenordnung von $V$. Da für die in Kapitel 4 vorgestellten Resultate $V$ mindestens $350 \mathrm{~V}$ betrug, folgt somit für die Oxidationsreaktion (6.7) unter dem Einfluss des elektrischen Feldes ein zusätzlicher Energiegewinn von etwa $700 \mathrm{eV}$. Thermodynamisch ist die Reaktion von Wolfram mit $\mathrm{H}_{2} \mathrm{O}$ somit auch bei starker Änderung der Aktivitäten der beteiligen Spezies stets bevorzugt. Für die Beobachtung, dass die Oxidationsreaktion in einem gewissen Stadium zum Erliegen kommt, kann es folglich nur kinetische Gründe geben.

\subsubsection{Identifikation des geschwindigkeitsbestimmenden Schrittes}

Nach den Betrachtungen aus 6.3.2 ist die Oxidationsreaktion von einem gewissen Stadium an kinetisch gehemmt. Der geschwindigkeitsbestimmende Schritt erfordert eine Mindestfeldstärke $E^{\text {min }}$, welche nach 6.3.1 eine deutliche Druckabhängigkeit aufweist. Im Folgenden werden die einzelnen Teilschritte der Oxidationsreaktion näher betrachtet, um den geschwindigkeitsbestimmenden Schritt zu identifizieren.

Für den Ort, an dem der geschwindigkeitsbestimmende Schritt abläuft, kommen drei Positionen in Betracht:

1. Das Volumenoxid

2. Die Metall-Oxid-Grenzfläche

3. Die Oxidoberfläche

Sofern der geschwindigkeitsbestimmende Schritt im Volumenoxid erfolgt, ist die Reaktion durch die Diffusion von Kationen oder Anionen limitiert. Eine Abschätzung des Interdiffusionskoeffizienten $\widetilde{D}$ kann auf Grundlage der Kinetik der Morphologieentwicklung erfolgen: Nach Abb. 4.5 bildet sich auf W-Spitzen bei einem Druck von 10 mbar $\mathrm{H}_{2} \mathrm{O}$ innerhalb von weniger als $1 \mathrm{~s}$ eine Oxidschicht mit einer Dicke $d=70 \mathrm{~nm}$. Dabei tritt nach 6.1.2 keine signifikante Oberflächendiffusion auf. Für den Interdiffusionskoeffizienten folgt somit $\widetilde{D} \approx \frac{1}{2 t} d^{2}>10^{-15} \mathrm{~m}^{2} / \mathrm{s}$. Der Interdiffusionskoeffizient bei $296 \mathrm{~K}^{9}$ liegt demnach in der gleichen Größenordnung wie der Selbstdiffusionskoeffizienten von Oxiden an ihrem Schmelzpunkt [Brow1980]. Das elektrische Feld bewirkt somit eine erhebliche Absenkung der Barrieren für die Volumendiffusion.

Die Diffusion durch das Oxid kann jedoch im Allgemeinen nicht der geschwindigkeitsbestimmende Schritt sein. Dies wird insbesondere am Ort verschwindender Oxiddicke $r_{\mathrm{v}}$ deutlich: Die lokale Morphologie an der Position $r_{\mathrm{v}}$ ist nahezu unabhängig vom $\mathrm{H}_{2} \mathrm{O}-$

\footnotetext{
${ }^{9}$ Die bei der Oxidationsreaktion freiwerdende Reaktionsenthalpie $\Delta H$ führt zu einer Erhöhung der Temperatur an der Probenoberfläche um $\Delta T$. Zur Abschätzung von $\Delta T$ wird ein eindimensionales Modell betrachtet: Bei einem Reaktionskoeffizienten von 1 ergibt sich die freiwerdende Energie pro Zeit und Fläche aus der Kinetik des idealen Gases zu $P^{\text {re }}=\zeta p_{\mathrm{H}_{2} \mathrm{O}}\left(2 \pi \mathrm{m}_{\mathrm{H}_{2} \mathrm{O}} \mathrm{k} T\right)^{-\frac{1}{2}} \Delta H$. Die pro Zeit und Fläche durch die Wärmeleitung der Probe über die Distanz $l$ abfließende Energie beträgt $P^{\mathrm{wl}}=\lambda l^{-1} \Delta T$. Dabei ist $\lambda$ die spezifische Wärmeleitfähigkeit. Im stationären Zustand folgt für die Temperaturerhöhung der Probenoberfläche $\Delta T=\zeta l \lambda^{-1}\left(2 \pi \mathrm{m}_{\mathrm{H}_{2} \mathrm{O}} \mathrm{k} T\right)^{-\frac{1}{2}} p_{\mathrm{H}_{2} \mathrm{O}} \Delta H$. Für eine Abschätzung von $\Delta T$ wird der maximale $\mathrm{H}_{2} \mathrm{O}$-Druck von 10 mbar und eine Länge $l$ von $1 \mu \mathrm{m}$ zugrunde gelegt. Mit der spezifischen Wärmeleitfähigkeit für Wolfram von $170 \mathrm{~W} /(\mathrm{Km}), 1<\zeta<10$ und den Enthalpiewerten für die Bildung von $\mathrm{WO}_{3}$ aus [Knac1991] folgt eine Temperaturerhöhung von weniger als 0,1 K. Auch bei zusätzlicher Berücksichtigung der Energie, die bei der getriebenen Diffusion der Ionen durch das Oxid frei wird, steigt die Temperaturerhöhung maximal um einen Faktor 10. Die Oxidationsreaktion kann somit als isotherm betrachtet werden.
} 
Druck. Darüber hinaus kann nach 6.3.1 die Oxidationsreaktion an diesem Ort lokal als eindimensionales Problem betrachtet werden. Mit der Änderung der Permittivität um den Faktor $\varepsilon_{\mathrm{r}}$ beim Übergang vom Gasraum in das Oxid ändert sich die Feldstärke um den Faktor $\frac{1}{\varepsilon_{\mathrm{r}}}$. Aus der deutlichen Druckabhängigkeit von $E_{r_{\mathrm{v}}}^{\mathrm{g}}$ (siehe Abb. 4.17) folgt daher auch, dass die Feldstärke im Oxid deutlich vom $\mathrm{H}_{2} \mathrm{O}$-Druck abhängt. Da somit bei ähnlicher lokaler Morphologie die Oxidationsreaktion bei einer deutlich druckabhängigen Feldstärke im Oxid zum Erliegen kommt, kann Diffusion durch das Oxid nicht der geschwindigkeitsbestimmende Schritt sein. Auch bei elektrolytischer Oxidation von Wolfram wird bei einer Feldstärke im Oxid von $0,5 \mathrm{~V} / \mathrm{nm}$ noch eine Stromdichte in der Größenordnung von $10^{-18} \mathrm{~A} / \mathrm{nm}^{2}$ gemessen [Quar1980]. Es findet also noch Wachstum mit einer Rate von etwa $0,1 \mathrm{ML} / \mathrm{s}$ statt, im Gegensatz zum hier beobachteten Auftreten einer stabilen Morphologie.

Sollte der geschwindigkeitsbestimmende Schritt an der Metall-Oxid-Grenzfläche erfolgen, so ist die deutliche Druckabhängigkeit des für den Ablauf der Oxidationreaktion notwendigen elektrischen Feldes nur erklärbar, wenn sich auch die Stöchiometrie des gebildeten Oxides in Abhängigkeit vom $\mathrm{H}_{2} \mathrm{O}$-Druck ändert. Nach Abb. 4.12 und 4.3.2 wird jedoch keine signifikante Variation der Oxidstöchiometrie mit dem $\mathrm{H}_{2} \mathrm{O}$-Druck beobachtet. Sofern der geschwindigkeitsbestimmende Schritt an der Metall-Oxid-Grenzfläche erfolgt, sollte darüber hinaus die Oxidationsreaktion unabhängig von der Art des sauerstoffenthaltenden Gases ablaufen. Nach 5.4 wird jedoch für $\mathrm{O}_{2}$ nicht das gleiche Verhalten wie für $\mathrm{H}_{2} \mathrm{O}$ beobachtet. Die Oxidationsreaktion ist daher offenbar nicht durch einen an der Metall-Oxid-Grenzfläche ablaufenden Teilschritt begrenzt.

Der geschwindigkeitsbestimmende Schritt für die Oxidationsreaktion erfolgt somit an der Oxidoberfläche. Abhängig von der Lage der Reaktionsfront für die Reaktion zwischen Wolfram und Sauerstoff finden dabei unterschiedliche Reaktionen statt: Liegt die Reaktionsfront an der Oxidoberfläche, so verläuft die Reaktion gemäß

$$
\frac{1}{3} \mathrm{~W}^{6+}+\mathrm{H}_{2} \mathrm{O}(\mathrm{ad}) \rightarrow \frac{1}{3} \mathrm{WO}_{3}(\mathrm{~s})+2 \mathrm{H}^{+}(\mathrm{de}) \cdot{ }^{10}
$$

Sofern die Reaktionsfront nicht an der Oxidoberfläche liegt, ist Wolfram an der Oberflächenreaktion nicht beteiligt. Die an der Oxidoberfläche ablaufende Reaktion ist dann

$$
\mathrm{H}_{2} \mathrm{O}(\mathrm{ad}) \rightarrow \mathrm{O}^{2-}+2 \mathrm{H}^{+}(\mathrm{de}) \cdot{ }^{10}
$$

In beiden Fällen muß zum einen Sauerstoff für die Reaktion mit Wolfram zur Verfügung gestellt werden, zum anderen müssen H-Ionen gebildet werden, durch deren Desorption die Akkumulation positiver Ladung an der Oxidoberfläche verhindert wird. Beide Prozesse können daher geschwindigkeitsbestimmend für die Oxidationsreaktion sein. Nach [Akbu1995] und [Hend2002] erfolgt im feldfreien Fall reversible Dissoziation von $\mathrm{H}_{2} \mathrm{O}$ auf der Oxidoberfläche. Der geschwindigkeitsbestimmende Schritt für die Oxidationsreaktion ist demnach offenbar die Reaktion von Hydroxidionen an der Oxidoberfläche.

\footnotetext{
${ }^{10}$ Wie (6.7) sind auch (6.8) und (6.9) als Netto-Reaktionsgleichungen zu verstehen. Insbesondere kann durch die Frenkelreaktionen Null $\rightleftarrows \mathrm{W}^{6+}+[\mathrm{W}]^{6-}$ beziehungsweise $\mathrm{Null} \rightleftarrows \mathrm{O}^{2-}+[\mathrm{O}]^{2+}$ eine gleichwertige Formulierung mit Leerstellen anstelle von interstitiellen Atomen erfolgen.
} 


\subsection{Variation von Spitzenmaterial und Gasspezies}

In diesem Abschnitt wird diskutiert, wie im Rahmen des Modells für die feldinduzierte Tieftemperaturoxidation das unterschiedliche Oxidationsverhalten verschiedener Spitzenmaterialien verstanden werden kann. Dadurch kann der Einfluss von Materialeigenschaften auf die Oxidationsreaktion verdeutlicht werden. Des Weiteren wird diskutiert, weshalb an reiner Sauerstoffatmosphäre keine Oxidationsreaktion wie bei Anwesenheit von $\mathrm{H}_{2} \mathrm{O}$ beobachtet wird.

\subsubsection{Abhängigkeit der Oxidationsreaktion vom Spitzenmaterial}

Nach dem in diesem Kapitel abgeleiteten Modell für die feldinduzierte Tieftemperaturoxidation sind vier Materialeigenschaften wesentlich für den Ablauf der Oxidationsreaktion: Die Permittivität des Oxides ist ein Maß dafür, wie stark der Einfluss des elektrischen Feldes auf die Diffusion im Oxid ist. Zusammen mit der Höhe der Energiebarrieren für den Anionen- und Kationentransport beeinflusst sie maßgeblich den Diffusionsstrom. Die Bindungsenergie von $\mathrm{H}_{2} \mathrm{O}$ auf der Oxidoberfläche wirkt sich auf die Konzentration und damit auf den Aggregatzustand von $\mathrm{H}_{2} \mathrm{O}$ auf der Oxidoberfläche aus. Schließlich ist durch die Feldverdampfungsfeldstärke des Oxides eine Obergrenze für die an der Oxidoberfläche auftretende Feldstärke gegeben.

Die Ausbildung von Oxidschichten kann demnach nur beobachtet werden, sofern die Feldverdampfungsfeldstärke des Oxides höher ist als diejenige Feldstärke, die für den Ablauf des geschwindigkeitsbestimmenden Schrittes notwendig ist. Andernfalls geht die Bildung des Oxides unmittelbar mit dessen Feldverdampfung einher. ${ }^{11}$ Da durch den Einfluss des elektrischen Feldes die Reaktion zwischen Metall beziehungsweise Halbleiter und $\mathrm{H}_{2} \mathrm{O}$ thermodynamisch stets begünstigt ist (siehe 6.3.2), sollte feldinduzierte Oxidation für eine große Zahl von Elementen beobachtbar sein. Dabei ist jedoch nicht garantiert, dass die gebildeten Oxide stabil sind.

Vor dem Hintergrund dieser erwarteten Abhängigkeiten der Oxidationsreaktion von den Eigenschaften des Spitzenmaterials werden im Folgenden die Resultate für unterschiedliche Spitzenmaterialien diskutiert. Da die Resultate an W-Spitzen bereits zu Beginn dieses Kapitels ausführlich behandelt wurden, werden dabei nur die übrigen Materialien betrachtet.

Aluminium An Al-Spitzen wird in Abhängigkeit von der Reaktionszeit und vom $\mathrm{H}_{2} \mathrm{O}$-Druck eine ähnliche Morphologieänderung beobachtet wie an W-Spitzen. Nach [Fehl1970, S. 84] liegen bei Oxidation von Aluminium die Transportzahlen von Kationen und Anionen in der gleichen Größenordnung. Die Permittivität von $\mathrm{Al}_{2} \mathrm{O}_{3}$ beträgt nach [Bire1977, Prat1969] etwa 10. Somit sind diejenigen Materialeigenschaften, welche die Spitzenmorphologie wesentlich bestimmen, ähnlich wie bei Wolfram. Daher ist eine vergleichbare Morphologieentwicklung wie bei Wolfram zu erwarten.

Für die Feldstärke an der Oxidoberfläche am Ort verschwindender Oxiddicke $E_{r_{\mathrm{v}}}^{\mathrm{g}}$ wird bei Aluminium im Druckbereich von $10^{-4}$ mbar bis 10 mbar $\mathrm{H}_{2} \mathrm{O}$ eine ähnliche Druckabhängigkeit wie bei Wolfram beobachtet. Die Werte bei Aluminium liegen jedoch stets

\footnotetext{
${ }^{11}$ Dabei ist zu berücksichtigen, dass nach den Ausführungen in 6.1.2 Felddesorption von unterstöchiometrischem Wolframoxid bereits bei geringeren Feldstärken erfolgen kann.
} 
höher. Da nach 6.3.3 der geschwindigkeitsbestimmende Schritt der Oxidationsreaktion an der Oxidoberfläche stattfindet, kann das beobachtete Verhalten nicht direkt aus Eigenschaften des Volumenoxides abgeleitet werden. Die Schmelztemperatur von $\mathrm{Al}_{2} \mathrm{O}_{3}$ ist jedoch etwa $40 \%$ höher ist als die von $\mathrm{WO}_{3}$, daher ist nach [Brow1980] für $\mathrm{Al}_{2} \mathrm{O}_{3}$ auch eine entsprechend höhere Aktivierungsenthalpie für die Diffusion im feldfreien Fall zu erwarten. Sofern sich diese auch auf die Aktivierungsenthalpie für die Oberflächenreaktion auswirkt, etwa beim Übergang von Sauerstoff von der Oxidoberfläche in das Volumenoxid, ist der höhere Wert von $E_{r_{\mathrm{v}}}^{\mathrm{g}}$ bei Aluminium verständlich.

Ein deutlicher Einfluss eines elektrischen Feldes auf die Oxidation von Aluminium bei einem Druck von $7 \cdot 10^{-4}$ mbar $\mathrm{H}_{2} \mathrm{O}$ wird auch von Ebinger und Yates [Ebin1998] beobachtet. Das elektrische Feld über das Oxid hinweg entsteht in ihrer Studie durch Elektronenbestrahlung der Oxidoberfläche. Dadurch erhöht sich die Oxidationsrate erheblich. Bei einer Oxiddicke von mehr als 2,5 nm sinkt die Wachstumsrate jedoch stark ab. Ein Anstieg der Oxidationsrate wird somit nur bis zum Erreichen derjenigen Oxiddicke beobachtet, die sich bei feldfreier Oxidation von Aluminium an Laborluft mit einer üblichen Luftfeuchtigkeit einstellt [Do1997]. Als Ursache für das starke Absinken der Oxidationsrate geben Ebinger und Yates die Ausheilung von flächenhaften Defekten oder die Bildung von stöchiometrischem Aluminiumoxid an. In der hier vorgestellten Studie können demgegenüber erheblich dickere Oxidschichten erzeugt werden (siehe Abb. 5.5). Nach der Interpretation von Ebinger und Yates erfolgt das beschleunigte Wachstum der Oxidschicht unter einem konstanten Feld im Oxid in Höhe von $0,5 \mathrm{~V} / \mathrm{nm}$. Bei einer Permittivität $\varepsilon_{\mathrm{r}}=10$ entspricht dies derjenigen Feldstärke $E_{r_{\mathrm{v}}}^{\mathrm{g}}$, die nach Abb. 5.7 für einen Druck von $7 \cdot 10^{-4}$ mbar $\mathrm{H}_{2} \mathrm{O}$ zu erwarten ist. Die Ergebnisse der vorliegenden Studie deuten somit darauf hin, dass Volumentransport nicht geschwindigkeitsbestimmend für die Oxidationsreaktion ist.

Silizium Auch an Si-Spitzen wird feldinduzierte Oxidation in Übereinstimmung mit zahlreichen Studien [Garc1998, Mori1997, Stié1997, Teus1995] erst oberhalb einer gewissen Mindestfeldstärke beobachtet. Der Betrag der Feldstärke lässt sich nach [Nowa2006] aus der Abhängigkeit $r_{\mathrm{v}}^{2}\left(V^{2}\right)$ ermitteln. Mit den Daten aus Abb. 5.9 folgt $E_{r_{\mathrm{v}}}^{\mathrm{g}}=3,6(2) \mathrm{V} / \mathrm{nm}$. Die Mindestfeldstärke für die Oxidationsreaktion von Silizium an Luft, also bei einem Partialdruck von $\mathrm{H}_{2} \mathrm{O}$ in der Größenordnung von 10 mbar, liegt somit erheblich oberhalb der zur Oxidation von Wolfram und Aluminium notwendigen Feldstärken. Nach Abb. 6.8 ist jedoch davon auszugehen, dass bei dieser Feldstärke bereits Kondensation von $\mathrm{H}_{2} \mathrm{O}$ auf der Spitze stattfindet. Dann ist die wirksame Feldstärke geringer als die berechnete, da bei der Berechnung die Permittivität des $\mathrm{H}_{2} \mathrm{O}$ unberücksichtigt bleibt.

Molybdän, Nickel und Silber An Spitzen aus Molybdän wurde qualitativ das gleiche Oxidationsverhalten beobachtet wie an W-Spitzen. Da sich beide Elemente chemisch stark ähneln, ist ein solches Verhalten auch zu erwarten.

An Spitzen aus Nickel konnte keine feldinduzierte Oxidbildung beobachtet werden. Oberhalb einer kritischen Feldstärke tritt jedoch ein Verlust von Spitzenmaterial auf, wodurch sich der Krümmungsradius der Spitzen vergrößert. Die Ursache für den Materialverlust ist offenbar Feldverdampfung von Nickel oder Nickeloxid. Nickel besitzt nach [Mill1996, S. 492] keine auffällig geringe Feldverdampfungsfeldstärke, daher ist Feldver- 
dampfung von Nickel nicht anzunehmen. Nickeloxid hingegen hat nach [Fehl1986, S. 116] als einziges der untersuchten Oxide eine vergleichsweise niedrige M-O-Bindungsenergie (1,2 eV für Ni gegenüber etwa 4,5 eV für W, Al und Si). Daher folgt vermutlich unmittelbar auf die Bildung von Nickeloxid dessen Feldverdampfung, so dass keine Ausbildung von Oxidschichten beobachtet werden kann.

Die Ausbildung von Oxidschichten auch auf Ag-Spitzen zeigt, dass selbst Oxide, für deren Bildung die thermodynamische Triebkraft im feldfreien Fall gering ist, unter dem Einfluss eines externen elektrischen Feldes gebildet werden können. Der Prozess der feldinduzierten Oxidation ist somit weitgehend materialunabhängig.

\subsubsection{Abhängigkeit der Oxidationsreaktion von der Gasspezies}

Im feldfreien Fall ist die thermodynamische Triebkraft für die Oxidbildung mit $\mathrm{O}_{2}$ größer als die Triebkraft für die Oxidbildung mit $\mathrm{H}_{2} \mathrm{O}$. Dennoch wurde unter reiner $\mathrm{O}_{2}$ Atmosphäre an W-Spitzen keine feldinduzierte Oxidation wie unter $\mathrm{H}_{2} \mathrm{O}$-Atmosphäre oder an Umgebungsatmosphäre beobachtet. Die Ursache für dieses Verhalten liegt vermutlich in der Notwendigkeit, im Verlauf der Oxidationsreaktion eine Akkumulation positiver Ladung an der Oxidoberfläche zu verhindern.

Bei Reaktion mit $\mathrm{H}_{2} \mathrm{O}$ kann durch Desorption von $\mathrm{H}^{+}$-Ionen genau diejenige Ladungsmenge von der Oxidoberfläche entfernt werden, welche durch die Oxidationsreaktion von der Metall-Oxid-Grenzfläche zur Oxidoberfläche transportiert wird. Dies gilt unabhängig vom gebildeten Oxid, da Sauerstoff sowohl in $\mathrm{H}_{2} \mathrm{O}$ als auch in Oxiden stets die Oxidationszahl -II hat.

Bei Reaktion mit $\mathrm{O}_{2}$ wäre zum Ladungsausgleich eine Aufspaltung $\mathrm{O}_{2} \rightarrow \mathrm{O}^{2-}+\mathrm{O}^{2+}$ mit anschließender Desorption von $\mathrm{O}^{2+}$ notwendig. Eine solche Reaktion scheint aus Symmetriegründen jedoch kaum denkbar. Die Alternative einer Ladungskompensation durch Elektronentransport von der Metall-Oxid-Grenzfläche zur Oxidoberfläche ist ebenfalls unwahrscheinlich: Selbst beim Vorliegen geeigneter Leitungsmechanismen (siehe 2.2.1) müsste der Elektronentransport gegen das elektrische Feld erfolgen. Auch bei Existenz tiefliegender Akzeptorniveaus auf der Oxidoberfläche ist bei Feldstärken im Oxid von einigen zehntel $\mathrm{V} / \mathrm{nm}$ ein Elektronentransport an die Oxidoberfläche daher nicht mit einem Energiegewinn verbunden.

Fehlende Ladungskompensation ist somit die Ursache dafür, dass die Oxidationsreaktion unter $\mathrm{O}_{2}$-Atmosphäre nicht wie unter $\mathrm{H}_{2} \mathrm{O}$-Atmosphäre oder an Umgebungsatmosphäre erfolgt. Diese Interpretation ist dabei ein weiterer Beleg dafür, dass der geschwindigkeitsbestimmende Schritt für die Oxidationsreaktion tatsächlich an der Oxidoberfläche abläuft.

\subsection{Vergleich mit Modellen für Tieftemperaturoxidation aus der Literatur}

Bei der Kinetik der feldinduzierten Tieftemperaturoxidation von Wolfram können nach Abb. 4.4 und Abb. 6.3 zwei Regime unterschieden werden: Nach einem anfänglichen starken Anstieg von $r_{\mathrm{v}}$ beziehungsweise $\Delta z$ kommt die Oxidationsreaktion für hohen und 
niedrigen $\mathrm{H}_{2} \mathrm{O}$-Druck von einem gewissen Stadium an zum Erliegen. Bei feldfreier ${ }^{12}$ Tieftemperaturoxidation treten nach 2.2.2 üblicherweise ebenfalls zwei Regime auf. Für die einzelnen Regime wird im Folgenden die im Rahmen dieser Arbeit entwickelte Modellvorstellung zur feldinduzierten Tieftemperaturoxidation mit bekannten Modellen zur Tieftemperaturoxidation verglichen:

Die erhöhte Wachstumsrate im ersten Regime wird stets auf Diffusion unter dem Einfluss eines elektrischen Feldes zurückgeführt. Bei feldfreier Oxidation entsteht das elektrische Feld durch die Potentialdifferenz $V_{\text {Mott }}$ zwischen dem Fermi-Niveau des Metalls und Akzeptor-Niveaus des an der Oxidoberfläche chemisorbierten Sauerstoffs gemäß dem Modell von Cabrera und Mott [Cabr1949, Mott1947]. Bei feldinduzierter Oxidation wird das elektrische Feld von außen angelegt. Bei geringem $\mathrm{H}_{2} \mathrm{O}$-Druck ist die Kinetik der Reaktion dabei jedoch nicht durch die Diffusion, sondern durch den Antransport von $\mathrm{H}_{2} \mathrm{O}$ begrenzt.

Das starke Absinken der Oxidationsrate im zweiten Regime ist nach [Mott1940] auf einen drastisch abnehmenden Tunnelstrom von Elektronen durch die Oxidschicht zurückzuführen. Wegen des fehlenden Ladungsausgleiches zwischen Metall-Oxid-Grenzfläche und Oxidoberfläche kommt die Oxidationsreaktion daher zum Erliegen. Bei der feldinduzierten Tieftemperaturoxidation hingegen ist kein Elektronentransport durch das Oxid notwendig, da eine Akkumulation positiver Ladung an der Oxidoberfläche durch Desorption von $\mathrm{H}^{+}$-Ionen verhindert wird. Ursache für den extremen Abfall der Oxidationsrate ist hier das Unterschreiten einer kritischen Feldstärke, die für den Ablauf einer Reaktion von Hydroxidionen an der Oxidoberfläche notwendig ist. Die Existenz einer kritischen Feldstärke wird auch bei Oxidationsuntersuchungen an Silizium mit einem AFM bei anliegender Potentialdifferenz zwischen Spitze und Probe beobachtet [Garc1998, Mori1997, Stié1997, Teus1995].

\footnotetext{
${ }^{12} \mathrm{Als}$ feldfrei wird hier eine Oxidationsreaktion bezeichnet, welche ohne Einfluss eines externen elektrischen Feldes abläuft. Interne elektrische Felder können dabei jedoch auftreten.
} 



\section{Zusammenfassung}

Gegenstand der vorliegenden Arbeit war die Untersuchung des Oxidationsverhaltens nanoskaliger Spitzen unter dem Einfluss eines elektrischen Feldes. Durch den kombinierten Einsatz von Transmissionselektronenmikroskopie, Laser-unterstützter Atomsondentomographie und Berechnung der Feldverteilung mit der Finite-Elemente-Methode konnten wesentliche Mechanismen der Oxidationsreaktion offengelegt werden:

- Unter dem Einfluss eines elektrischen Feldes ändert sich das Oxidationsverhalten nanoskaliger Spitzen aus Wolfram erheblich, sofern $\mathrm{H}_{2} \mathrm{O}$ für die Reaktion zur Verfügung steht. Unter reiner $\mathrm{O}_{2}$-Atmosphäre wird keine vergleichbare Reaktion beobachtet.

- Für den Ablauf der Oxidationsreaktion ist eine vom Druck abhängige Mindestfeldstärke in der Größenordnung von $1 \mathrm{~V} / \mathrm{nm}$ notwendig. Mit abnehmendem $\mathrm{H}_{2} \mathrm{O}$ Partialdruck steigt die Mindestfeldstärke gemäß Abb. 4.17 monoton an. Wird die Mindestfeldstärke unterschritten, so kommt die Oxidationsreaktion im Rahmen der experimentellen Genauigkeit zum Erliegen.

- Bei der feldinduzierten Oxidation wird die Ausbildung von Oxidschichten mit einer Dicke von einigen $10 \mathrm{~nm}$ beobachtet. Die Morphologie der nanoskaligen Spitzen samt Oxid weist dabei eine deutliche Abhängigkeit vom $\mathrm{H}_{2} \mathrm{O}$-Druck auf. Mit abnehmendem Druck wird ein Übergang von flächenhaften Oxidschichten zu kegelförmigen Oxidschichten beobachtet (siehe Abb. 4.6). Die Druckabhängigkeit der Spitzenmorphologie kann durch das Zusammenwirken von Oberflächendiffusion, Feldverdampfung und Volumendiffusion verstanden werden.

- Die chemische Charakterisierung der Oxidschichten mit Laser-unterstützter Atomsondentomographie zeigt, dass leicht unterstöchiometrisches $\mathrm{WO}_{3}$ gebildet wird. Die Stöchiometrie ist dabei unabhängig vom $\mathrm{H}_{2} \mathrm{O}$-Druck während der Oxidationsreaktion. Die Oxidschichten sind nanokristallin.

- Die Reaktionsgleichung für die Oxidationsreaktion bei Anwesenheit von $\mathrm{H}_{2} \mathrm{O}$ lautet $\frac{1}{3} \mathrm{~W}(\mathrm{~s})+\mathrm{H}_{2} \mathrm{O}(\mathrm{g}) \rightarrow \frac{1}{3} \mathrm{WO}_{3}(\mathrm{~s})+2 \mathrm{e}^{-}+2 \mathrm{H}^{+}$. Die Elektronen werden beim Übergang des Wolframs in das Oxid frei, die $\mathrm{H}^{+}$-Ionen entstehen bei der Reaktion des $\mathrm{H}_{2} \mathrm{O}$ an der Oxidoberfläche. Durch Desorption der $\mathrm{H}^{+}$-Ionen wird eine Akkumulation positiver Ladung an der Oxidoberfläche verhindert. Ein Ionenstrom der entsprechenden Größe kann experimentell nachgewiesen werden.

- Thermodynamisch ist der Ablauf der feldinduzierten Oxidationsreaktion unter den beschriebenen experimentellen Bedingungen stets begünstigt. Die Existenz einer für den Ablauf der Reaktion notwendigen Mindestfeldstärke weist somit darauf hin, dass die Reaktion von einem gewissen Stadium an kinetisch gehemmt ist.

- Die Energiebarrieren für die Diffusion der Reaktionspartner durch die Oxidschicht werden durch das elektrische Feld soweit abgesenkt, dass Volumendiffusion nicht 
geschwindigkeitsbestimmend für die Oxidationsreaktion ist. Die Oxidationsreaktion kommt zum Erliegen, sobald die Feldstärke an der Oxidoberfläche nicht mehr hinreichend hoch ist, um eine Reaktion von Hydroxidionen zu aktivieren. Für einen $\mathrm{H}_{2} \mathrm{O}$-Druck von weniger als $10^{-3}$ mbar ist die Kinetik der Reaktion zuvor durch den Antransport von $\mathrm{H}_{2} \mathrm{O}$ begrenzt.

- Die Ausbildung von Oxidschichten unter dem Einfluss eines Feldes wird nicht nur an Spitzen aus Wolfram, sondern auch an Spitzen aus Aluminium, Silizium, Molybdän und Silber beobachtet. Feldinduzierte Oxidation ist somit ein weitgehend materialunabhängiger Prozess. 


\section{Symbolverzeichnis}

$\chi_{\mathrm{i}} \quad$ Dosis der Spezies i

$\Delta G \quad$ Änderung der Gibbsschen freie Energie

$\Delta G^{0} \quad$ Änderung der Gibbsschen freien Energie unter Standarddruck

$\Delta H \quad$ Änderung der Enthalpie

$\Delta z \quad$ Betrag der Längenänderung der metallischen Spitze auf der Symmetrieachse

$\eta \quad$ Koordinate im parabolischen Koordinatensystem

$\lambda \quad$ spezifische Wärmeleitfähigkeit

$\mu_{\mathrm{i}} \quad$ chemisches Potential der Spezies i

$\mu_{\mathrm{i}}^{0} \quad$ chemisches Potential der Spezies i unter Standarddruck

$\nu \quad$ Frequenz der thermischen Schwingungen eines Moleküls bzw. Ions um seine Ruhelage

$\Phi \quad$ elektrostatisches Potential

$\rho_{\mathrm{i}} \quad$ Dichte der Substanz i

$\theta^{\mathrm{v}}, \theta^{\mathrm{r}} \quad$ Flächendichte mobiler Ionen für Vor- und Rücksprünge

$\theta_{\mathrm{i}} \quad$ Flächendichte von Molekülen der Spezies i

$\varepsilon_{0} \quad$ elektrische Feldkonstante

$\varepsilon_{\mathrm{r}} \quad$ Permittivitätszahl

$\varphi \quad$ Winkelkoordinate in Zylinderkoordinaten

$\widetilde{\mathrm{M}}_{\mathrm{i}} \quad$ Molmasse der Spezies i

$\widetilde{\mathrm{V}}_{\mathrm{i}} \quad$ Volumen der Elementarzelle der Spezies i

$\widetilde{D} \quad$ Interdiffusionskoeffizient

$\widetilde{V}_{\mathrm{M}} \quad$ Volumen des im Verlauf der Oxidationsreaktion aufgezehrten Metalls oder Halbleiters

$\widetilde{V}_{\mathrm{O}} \quad$ Volumen des Oxides auf der Spitze

$\xi \quad$ Koordinate im parabolischen Koordinatensystem

$\zeta \quad$ Faktor, um den sich die Auftreffrate eines Gases unter dem Einfluss eines Feldes erhöht

A Aktivierungsenergie für Diffusion im feldfreien Fall 
a halber Abstand zweier benachbarter Minima in der Potentiallandschaft

D dielektrische Verschiebung

d Dicke der Oxidschicht auf der Symmetrieachse

E Betrag der elektrischen Feldstärke

$E^{\text {min }} \quad$ Mindestfeldstärke für den Ablauf der Oxidationsreaktion

$E_{\mathrm{o}} \quad$ Betrag der Feldstärke an der Spitzenoberfläche

$E_{\mathrm{o}}^{\min }$ für die Oxidationsreaktion notwendige Mindestfeldstärke an der Oxidoberfläche

$E_{r_{\mathrm{V}}}^{\mathrm{g}} \quad$ Betrag der elektrischen Feldstärke an der Oxidoberfläche im Gasraum am Ort verschwindender Oxiddicke $r_{\mathrm{v}}$

F $\quad$ Fläche

$h \quad$ Oxidschichtdicke in Planarsystemen

I Während der Oxidationsreaktion an der Gegenelektrode gemessener Strom

$I_{\mathrm{p}} \quad$ Plateauwert des Stromes während der Oxidationsreaktion

$j_{\mathrm{i}} \quad$ Teilchenstromdichte der Spezies i

l Distanz

$P \quad$ Leistung pro Fläche

$p_{\mathrm{i}} \quad$ Partialdruck der Spezies i

$R \quad$ Verhältnis von Oxidvolumen zum Volumen des aufgezehrten Metalls oder Halbleiters

$r \quad$ radial Koordinate in Zylinderkoordinaten

$r_{\mathrm{v}} \quad$ Radius der Spitze am Ort verschwindender Oxiddicke

T absolute Temperatur

$t \quad$ Zeit

$t_{\mathrm{s}} \quad$ Zeit, für die während der Oxidationsreaktion ein Strom messbar ist

$t_{r_{\mathrm{v}}} \quad$ Zeit die vergeht, bis $r_{\mathrm{v}}$ einen festen Wert annimmt

$V \quad$ Potentialdifferenz zwischen Spitze und Gegenelektrode

$V_{\text {Mott }}$ Potentialdifferenz zwischen dem Fermi-Niveau des Metalls und Akzeptor-Niveaus des an der Oxidoberfläche chemisorbierten Sauerstoffs

z axiale Koordinate in Zylinderkoordinaten

$[\mathrm{M}]^{\mathrm{z}-}$ Leerstelle eines Atoms aus der Gruppe der Metalle oder Halbleiter

$[\mathrm{O}]^{\mathrm{z}+}$ Sauerstoff Leerstelle 


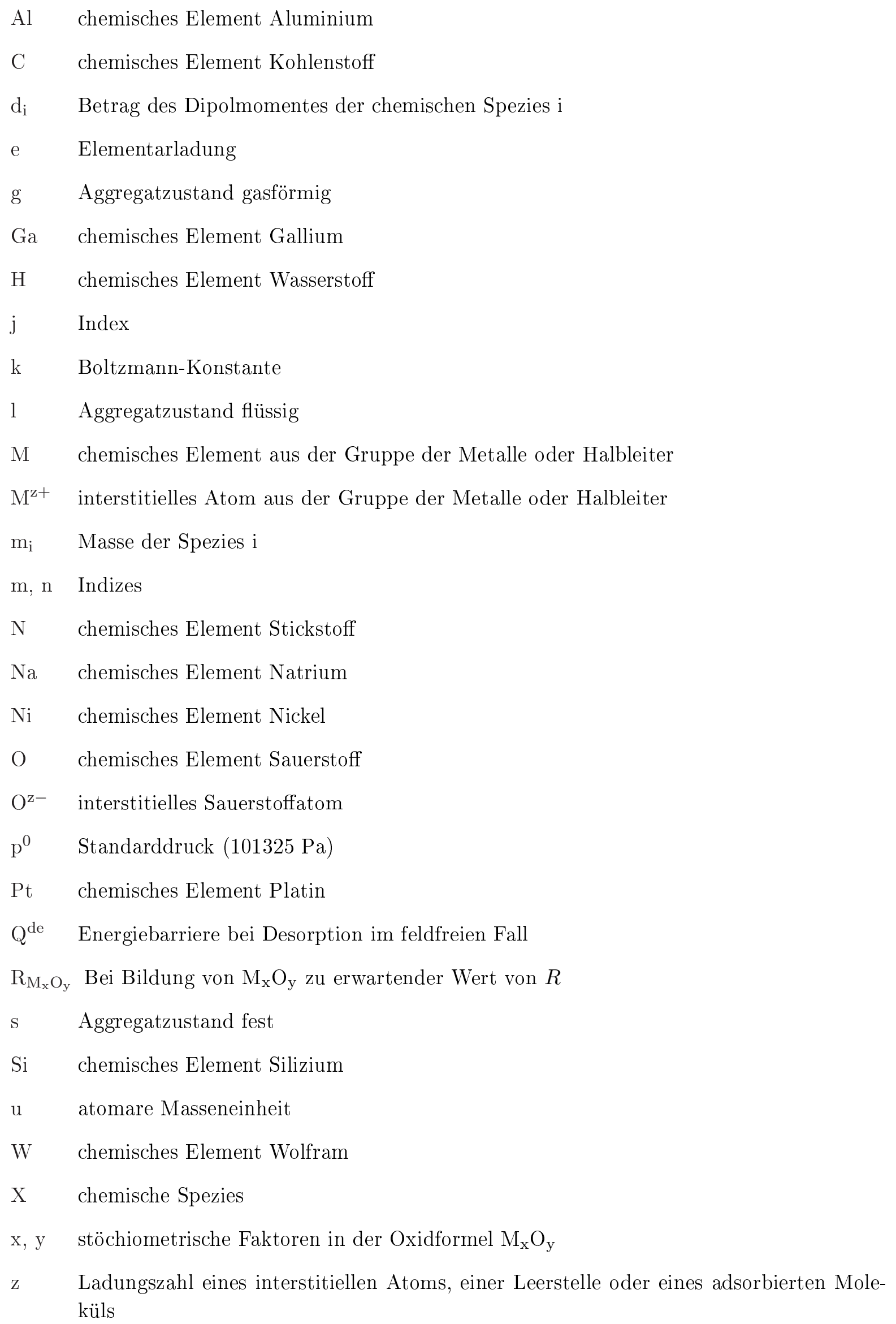





\section{Literaturverzeichnis}

[Akbu1995] Akbulut, M. ; Sack, N. J. ; Madey, T. E.: Adsorption and reaction of water on oxidized tungsten: thermal desorption and electron stimulated desorption measurements. In: Surf. Sci. 351 (1995), S. 209

[Ande1967] Anderson, J. R. ; Ritchie, I. M.: The Effect on a Tarnishing Reaction of an Electric Field Across the Growing Product Layer. In: Proc. R. Soc. London A 299 (1967), S. 371

[Avou1997] Avouris, P. ; Hertel, T. ; Martel, R.: Atomic force microscope tip-induced local oxidation of silicon: kinetics, mechanism, and nanofabrication. In: Appl. Phys. Lett. 71 (1997), S. 285

[Barr1978] BARR, T. L.: An ESCA Study of the Termination of the Passivation of Elemental Metals. In: J. Phys. Chem. 82 (1978), S. 1801

[Bire1977] BiREy, H.: Thickness dependence of the dielectric constant and resistance of $\mathrm{Al}_{2} \mathrm{O}_{3}$ films. In: J. Appl. Phys. 48 (1977), S. 5209

[Bogg1970] Boggio, J. E.: Formation of Very Thin Oxide Films on Metals: Contact Potential Measurements during the Oxidation of (100) Cu. In: J. Chem. Phys. 53 (1970), S. 3544

[Brow1980] Brown, A. M. ; Ashby, M. F.: Correlations for Diffusion Constants. In: Acta Metall. 28 (1980), S. 1085

[Cabr1949] Cabrera, N. ; Mott, N. F.: Theory of the oxidation of metals. In: Rep. Prog. Phys. 12 (1949), S. 163

[Cui2001] Cui, Y. ; Wei, Q. ; PARK, H. ; Lieber, C. M.: Nanowire Nanosensors for Highly Sensitive and Selective Detection of Biological and Chemical Species. In: Science 293 (2001), S. 1289

[Daga1998] Dagata, J. A. ; Inoue, T. ; Iтоh, J. ; Yokoyama, H.: Understanding scanned probe oxidation of silicon. In: J. Appl. Phys. 73 (1998), S. 271

[Davi1965] Davies, J. A. ; Domeij, B. ; Pringle, J. P. S. ; Brown, F.: The Migration of Metal and Oxygen during Anodic Film Formation. In: J. Electrochem. Soc. 112 (1965), S. 675

[Djur2007] Duurkovic, S. ; Clemons, C. B. ; Golovaty, D. ; Young, G. W.: Effects of the electric field shape on nano-scale oxidation. In: Surf. Sci. 601 (2007), S. 5340

[Do1997] Do, T. ; Splinter, S. J. ; Chen, C. ; MCIntyre, N. S.: The oxidation kinetics of $\mathrm{Mg}$ and Al surfaces studied by AES and XPS. In: Surf. Sci. 387 (1997), S. 192 
[Dubo2000] Dubois, E. ; Bubendorff, J.-L.: Kinetics of scanned probe oxidation: Spacecharge limited growth. In: J. Appl. Phys. 87 (2000), S. 8148

[Ebin1998] Ebinger, H. D. ; YATES JR., J. T.: Electron-impact-induced oxidation of Al(100) in water vapor: Relation to the Cabrera-Mott mechanism. In: Phys. Rev. B 57 (1998), S. 1976

[Eeke1970] Eekelen, H. A. M. van: The Behaviour of the Field-Ion Microscope: A Gas Dynamical Calculation. In: Surf. Sci. 21 (1970), S. 21

[Elli1944] Ellingham, H. J. T.: Reducibility of Oxides and Sulfides in Metallurgical Processes. In: J. Soc. Chem. Ind. 63 (1944), S. 125

[Ene2008] Ene, C.-B. ; Nowak, C. ; Schmitz, G.: Stress Impact on Reactive Diffusion in Nano-Structures of Spherical Symmetry. In: Solid State Phenom. 138 (2008), S. 367

[Enta2008] Enta, Y. ; Mun, B. S. ; Rossi, M. ; Ross JR., P. N. ; Hussain, Z. ; Fadley, C. S. ; LEE, K.-S. ; KIM, S.-K.: Real-time observation of the dry oxidation of the $\mathrm{Si}(100)$ surface with ambient pressure x-ray photoelectron spectroscopy. In: Appl. Phys. Lett. 92 (2008), S. 012110

[Fehl1970] Fehlner, F. P. ; Mott, N. F.: Low-Temperature Oxidation. In: Oxid. Met. 2 (1970), S. 59

[Fehl1986] Fehlner, F. P.: Low-Temperature oxidation. The Role of Vitreous Oxides. New York : John Wiley \& Sons, 1986

[From1976] Fromhold JR., A. T.: Theory of Metal Oxidation. Volume I - Fundamentals. Amsterdam : North-Holland Publishing Company, 1976

[From1998] Fromm, E.: Kinetics of Metal-Gas Interactions at Low Temperatures. Berlin : Springer, 1998

[Garc1998] García, R. ; Calleja, M. ; Pérez-Murano, F.: Local oxidation of silicon surfaces by dynamic force miroscopy: Nanofabrication and water bridge formation. In: Appl. Phys. Lett. 72 (1998), S. 2295

[Garc2006] García, R. ; Martínez, R. V. ; Martinez, J.: Nano-chemistry and scanning probe nanolithographies. In: Chem. Soc. Rev. 35 (2006), S. 29

[Gian2005] Giannuzzi, L. A. ; Geurts, R. ; Ringnalda, J.: $2 \mathrm{keV} \mathrm{Ga}{ }^{+}$FIB Milling for Reducing Amorphous Damage in Silicon. In: Microsc. Microanal. 11 (2005), S. 828

[Hann1983] Hanna, A. A. ; Khilla, M. A.: Electrical Properties of the Semiconductor Materials Molybdenum and Tungsten Trioxides. In: Thermochim. Acta 65 (1983), S. 311

[Hatt1994] Hattori, T. ; EjIRI, Y. ; SAito, K.: Fabrication of nanometer-scale structures using atomic force microscope with conducting probe. In: J. Vac. Sci. Technol. A 12 (1994), S. 2586 
[Hend2002] Henderson, M. A.: The interaction of water with solid surfaces: fundamental aspects revisited. In: Surf. Sci. Rep. 46 (2002), S. 1

[Hunt1970] Hunt, G. L. ; Ritchie, I. M.: The Effect of an Applied Field on the Oxidation of Aluminum in the Temperature Range $50-400^{\circ} \mathrm{C}$. In: Oxid. Met. 2 (1970), S. 361

[Jeur2002] Jeurgens, L. P. H. ; Sloof, W. G. ; Tichelaar, F. D. ; Mittemeijer, E. J.: Growth kinetics and mechanisms of aluminum-oxide films formed by thermal oxidation of aluminum. In: J. Appl. Phys. 92 (2002), S. 1649

[Jorg1962] Jorgensen, P. J.: Effect of an Electric Field on Silicon Oxidation. In: J. Chem. Phys. 37 (1962), S. 874

[King1971] King, D. A. ; Madey, T. E. ; Yates JR, J. T.: Interaction of Oxygen with Polycrystalline Tungsten. I. Sticking Probabilities and Desorption Spectra. In: $J$. Chem. Phys. 55 (1971), S. 3236

[Kirk1968] KIRK JR., C. T. ; Huber JR., E. E.: The Oxidation of Aluminum Films in Low-Pressure Oxygen Atmospheres. In: Surf. Sci. 9 (1968), S. 217

[Knac1991] Knacke, O. (Hrsg.) ; Kubaschewski, O. (Hrsg.) ; Hesselmann, K. (Hrsg.): Thermodynamical Properties of Inorganic Substances II. Berlin : Springer, 1991

[Kura2004] Kuramochi, H. ; Ando, K. ; Tokizaki, T. ; Yokoyama, H.: In situ detection of faradaic current in probe oxidation using a dynamic force microscope. In: Appl. Phys. Lett. 84 (2004), S. 4005

[Liu1994] Liu, H. I. ; Biegelsen, D. K. ; Ponce, F. A. ; Johnson, N. M. ; Pease, R. F. W.: Self-limiting oxidation for fabricating sub-5 nm silicon nanowires. In: Appl. Phys. Lett. 64 (1994), S. 1383

[Mart1994] Martin, M. ; Mader, W. ; Fromm, E.: Oxidation of iron, aluminium and titanium films in the temperature range $50-200^{\circ}$ C. In: Thin Solid Films 250 (1994), S. 61

[Mass1990] Massalski, T. B.: Binary alloy phase diagrams. Ohio : ASM International, 1990

[Mill1996] Miller, M. K. ; Cerezo, A.; Hetherington, M. G. ; Smith, G. D. W.: Atom Probe Field Ion Microscopy. Oxford : Oxford University Press, 1996

[Mill2005] Miller, M. K. ; Russell, K. F. ; Thompson, G. B.: Strategies for fabricating atom probe specimens with a dual beam FIB. In: Ultramicroscopy 102 (2005), S. 287

[Mori1997] Morimoto, K. ; Araki, K. ; Yamashita, K. ; Morita, K. ; Niwa, M.: Si nanofabrication using AFM field enhanced oxidation and anisotropic wet chemical etching. In: Appl. Surf. Sci. 117/118 (1997), S. 652

[Mott1940] Motт, N. F.: The Theory of the Formation of Protective Oxid Films on Metals, II. In: Trans. Faraday Soc. 35 (1940), S. 472

[Mott1947] Mотт, N. F.: The theory of the formation of protective oxide films on metals, III. In: Trans. Faraday Soc. 43 (1947), S. 429 
[Naka2007] Nakamura, R. ; Tokozakura, D. ; Nakajima, H. ; Lee, J.-G. ; Mori, H.: Hollow oxide formation by oxidation of $\mathrm{Al}$ and $\mathrm{Cu}$ nanoparticles. In: J. Appl. Phys. 101 (2007), S. 074303

[Nowa2006] Nowak, C. ; Kirchheim, R. ; Schmitz, G.: Electric-field-induced low temperature oxidation of tungsten nanowires. In: Appl. Phys. Lett. 89 (2006), S. 143104

[Ohno2005] Ohno, S. ; YATes JR., J. T.: Electron impact effects on the oxidation of Si(111) at 90 K. In: J. Vac. Sci. Technol. A 23 (2005), S. 475

[Pill1923] Pilling, N. B. ; Bedworth, R. E.: The Oxidation of Metals at High Temperatures. In: J. Inst. Met. 29 (1923), S. 529

[Poll1964] Pollack, S. R. ; Morris, C. E.: Electron Tunneling through Asymmetric Films of Thermally Grown $\mathrm{Al}_{2} \mathrm{O}_{3}$. In: J. Appl. Phys. 35 (1964), S. 1503

[Popo2002] Popova, I. ; Zhukov, V. ; Yates JR., J. T.: Electrostatic Field Enhancement of Al(111) Oxidation. In: Phys. Rev. Lett. 89 (2002), S. 276101-1

[Prat1969] PRATt, I. H.: Growth and electrical characteristics of r.f. sputtered aluminium oxide. In: Thin Solid Films 3 (1969), S. R23

[Pred1998] Predel, B.: Landolt-Börnstein: new series. group IV vol. 5 subvol. I. Berlin : Springer, 1998

[Quar1980] Quarto, F. D. ; C. Sunseri, A. D. P.: Kinetics of Growth of Amorphous $\mathrm{WO}_{3}$ Anodic Films on Tungsten. In: J. Electrochem. Soc. 127 (1980), S. 1016

[Ritc1970] Ritchie, I. M. ; Scott, G. H. ; Fensham, P. J.: The effect of an electric field on the oxidation rate of nickel between 250 and $380^{\circ}$ C. In: Surf. Sci. 19 (1970), S. 230

[Ross2008] Rossi, M. ; Mun, B. S. ; Enta, Y. ; FAdley, C. S. ; Lee, K.-S. ; Kim, S.-K. ; Shin, H.-J. ; Hussain, Z. ; Ross JR., P. N.: In situ observation of wet oxidation kinetics on $\mathrm{Si}(110)$ via ambient pressure x-ray photoemission scectroscopy. In: $J$. Appl. Phys. 103 (2008), S. 044104

[Sall2000] Sallés-Desvignes, I. ; Bertrand, G. ; Montesin, T. ; Favergeon, J.: Coupling between Diffusion, Stress Field and Chemical Reaction in a Metal-Gas Oxidation. In: Solid State Phenom. 72 (2000), S. 9

[Schm1964] SCHmidt, W. A.: Massenspektrometrische Untersuchung der Feldionisation von Wasserdampf an Spitzen aus Wolfram, Platin und Iridium. In: Z. Naturforsch. 19a (1964), S. 318

[Shos1991] Shostak, S. L. ; Ebenstein, W. L. ; Muenter, J. S.: The dipole moment of water. I. Dipole moments and hyperfine properties of $\mathrm{H}_{2} \mathrm{O}$ and $\mathrm{HDO}$ in the ground and excited vibrational states. In: J. Chem. Phys. 94 (1991), S. 5875

[Snow2000] Snow, E. S. ; Jernigan, G. G. ; Campbell, P. M.: The kinetics and mechanism of scanned probe oxidation of Si. In: Appl. Phys. Lett. 76 (2000), S. 1782 
[Sten2007] Stender, P. ; Oberdorfer, C. ; Artmeier, M. ; Pelka, P. ; Spaleck, F. ; Schmitz, G.: New tomographic atom probe at University of Muenster, Germany. In: Ultramicroscopy 107 (2007), S. 726

[Stié1997] Stiévenard, D. ; Fontaine, P. A. ; Dubois, E.: Nanooxidation using a scanning probe microscope: An analytical model based on field induced oxidation. In: Appl. Phys. Lett. 70 (1997), S. 3272

[Teus1995] Teuschler, T. ; Mahr, K. ; Miyazaki, S. ; Hundhausen, M. ; Ley, L.: Nanometer-scale field-induced oxidation of $\mathrm{Si}(111): \mathrm{H}$ by a conducting-probe scanning force microscope: Doping dependence an kinetics. In: Appl. Phys. Lett. 67 (1995), S. 3144

[Thie1987] Thiel, P. A. ; Madey, T. E.: The Interaction of Water with Solid Surfaces: Fundamental Aspects. In: Surf. Sci. Rep. 7 (1987), S. 211

[Vull1999] Vullers, R. J. M. ; Ahlskog, M. ; Cannaerts, M. ; Van Haesendonck, C.: Field induced local oxidation of $\mathrm{Ti}$ and $\mathrm{Ti} / \mathrm{Al}$ structures by an atomic force microscope with diamond coated tips. In: J. Vac. Sci. Technol. B 17 (1999), S. 2417

[Wagn1933] Wagner, C.: Beitrag zur Theorie des Anlaufvorgangs. In: Z. Phys. Chem. B 21 (1933), S. 25

[Walt2002] Walter, E. C. ; Penner, R. M. ; Liu, H. ; NG, K. H. ; Zach, M. P. ; Favier, F.: Sensors from electrodepoited metal nanowire. In: Surf. Interf. Anal. 34 (2002), S. 409

[Xie2006] XIE, X. N. ; Chung, H. J. ; Sow, C. H. ; Wee, A. T. S.: Nanoscale materials patterning and engineering by atomic force microscopy nanolithography. In: Mat. Sci. Eng. R 54 (2006), S. 1

[Xu1997] Xu, J. ; Choyke, W. J. ; Yates JR., J. T.: Enhanced silicon oxide film growth on Si (110) using electron impace. In: J. Appl. Phys. 82 (1997), S. 6289

[Yin2004] Yin, Y. ; Rioux, R. M. ; Erdonmez, C. K. ; Hughes, S. ; Somorjai, G. A. ; Alivisatos, A. P.: Formation of Hollow Nanocrystals Through the Nanoscale Kirkendall Effect. In: Science 304 (2004), S. 711

[Zhuk2002] Zhukov, V. ; Popova, I. ; Yates JR., J. T.: Electron-stimulated oxidation of $\mathrm{Al}(111)$ by oxygen at low temperatures: Mechanism of enhanced oxidation kinetics. In: Phys. Rev. B 65 (2002), S. 195409 



\section{Dank}

An der Entstehung dieser Arbeit haben zahlreiche Personen mitgewirkt, für deren Engagement ich mich an dieser Stelle bedanken möchte. Zunächst gilt mein besonderer Dank Herrn Prof. Dr. R. Kirchheim und Herrn Prof. Dr. G. Schmitz, die in zahlreichen Diskussionen Impulse für den Fortgang der Arbeit gaben. Ihr fortwährendes Interesse und die Freiräume, die sie mir gewährten, waren eine große Bereicherung. Herrn C. Oberdorfer vom Institut für Materialphysik der Universität Münster danke ich sehr für die weitreichende Unterstützung bei Messungen mit Laser-unterstützter Atomsondentomographie. Durch eine Vielzahl von Gesprächen und Anregungen habe zahlreiche Kollegen am Institut für Materialphysik der Universität Göttingen an der Entstehung dieser Arbeit mitgewirkt. Ihre Beiträge weiß ich sehr zu schätzen. Den Mitarbeitern der Werkstätten und aus dem Laborbereich bin ich dankbar für die technische Unterstützung bei Aufbau und Betrieb der experimentellen Anlagen. Schließlich seien noch die Mitarbeiter aus der Verwaltung und den Sekretariaten genannt, deren Sachkenntnis administrative Aufgaben stets erleichterte. 



\section{Lebenslauf}

\section{Persönliche Daten}

Carsten Nowak

Geburtsdatum: 14.12.1976

Geburtsort: Hildesheim

Nationalität: deutsch

\section{Studium und Schulbildung}

seit 2004 wissenschaftlicher Mitarbeiter am Institut für Materialphysik der Universität Göttingen

seit 2003 Dozent am XLAB - Experimentallabor für junge Leute e. V. in Göttingen

1999 - 2004 Hauptstudium der Physik an der Universität Göttingen, Abschluss Diplom

1999 - 2004 Studium der Politikwissenschaft und Pädagogik an der Universität Göttingen

1997 - 1999 Grundstudium der Physik an der Universität Göttingen

1983 - 1996 Schulbildung, Erwerb der Allgemeinen Hochschulreife am Gymnasium Nordenham 
